\title{
Operational Spacetimes
}

\author{
Heinrich Saller ${ }^{1}$
}

Received: 18 August 2016 / Accepted: 24 October 2016 / Published online: 1 December 2016 (c) The Author(s) 2017. This article is published with open access at Springerlink.com

\begin{abstract}
Time, positions and spacetimes are modelled by groups and subgroup classes. Their Hilbert space representations define particles and interactions. Electroweak spacetime, proposed as nature-realized manifold, represents the homogeneous groups of the electroweak standard model. The leptonic and hadronic (quark) sectors, characteristic for particles and interactions, are related to the embedded time and position operations, respectively.
\end{abstract}

Keywords Spacetime · Quantum fields

\section{Introduction}

This paper is dedicated to the memory of my friend David Finkelstein, of his scientific achievements, his prophetic strength and his personal modesty.

Quantum theory is a theory of operations. In his book "Quantum Relativity", ${ }^{1}$ David Finkelstein called the corresponding action-based philosophy "praxism, which renounces ontology and absolute states of being in favor of actions." In the foreword, Finkelstein writes: "... if you have seen how one uses quantum theory to predict the results of experiments, and still wonder what it all means; if you enjoy algebra; and if you can imagine that nature might permit a comprehensive theory and still not admit a complete one; then you are my ideal reader." This applies also to the following remarks.

Space and time and particles, or, with Bohr, possibly the whole conceptual apparatus of classical physics, are apparently necessary to talk about the experiments. However, after the

${ }^{1}$ D.R. Finkelstein, Quantum Relativity (1996), Springer, Berlin Heidelberg, New York.

Heinrich Saller

hns@mppmu.mpg.de

1 Max-Planck-Institut für Physik, Werner-Heisenberg-Institut, München, Germany 
labors and the birth of quantum theory with its "quantum relativity," i.e., with the different experimental projections of the operator formulation for a classical interpretation, the confidence in an all-encompassing objective fundamentality of those concepts is broken, at least in the Copenhagen interpretation.

Physics started as a science with four basic concepts - forces for matter in time and space. Those concepts can be seen as arising from an operational root: A formalization of physical laws is simultaneously a formalization of the laws of our experimental knowledge. Quantum information comes in the form of operation group coefficients. If physical dynamics is defined as the causal behavior of symmetry operations, then the concepts space and time, parametrizing these operations, and interactions and matter, implementing them, are "only" human related concepts for their description.

Basic interactions and particles are characterized by operation groups. Interactions and particles implement and parametrize space and time which, in the following, come as groups or equivalence classes of groups, e.g., Minkowski spacetime as Lorentz group classes of the Poincare group. In quantum theory, the concept of free particles as building bricks for bound states becomes fuzzy. For high energies, the flat spacetime implementation by particles seems to fade away as suggested by the quarks if they are really confined.

Interactions with particles as one concept and position with time as another concept cannot be separated; they cannot be thought of without each other. Einstein (1950): "According to general relativity, the concept of space detached from any physical content does not exist. The physical reality is represented by a field." And in 1954: "Space as opposed to "what fills space' which is dependent on the coordinates has no separate existence... There is no such thing as an empty space, i.e., a space without field." Or Born (1962): "It is not space that is there and that impresses its forms on things, but the things and their physical laws that determine space" — no spacetime without interactions and matter.

To clarify my attitude with respect to the description of basic physical structures by symmetries, I quote from the last public talk (1975) of Heisenberg Was ist ein Elementarteilchen (my translation):

It is unavoidable that we use a language originating from classical philosophy. We ask: What does the proton consist of? Is the quantum of light elementary or composite?, etc. However, all these questions are wrongly asked since the words "divide" and "consist of" have lost almost all their meaning. Therefore, it would be our task to adjust our language, our thinking, i.e., our scientific philosophy, to this new situation that has been created by the experiments. Unfortunately, that is very difficult. Therefore, there creep into particle physics, always again, wrong questions and wrong conceptions, ...

We have to come to terms with the fact that the experimental experiences for very small and for very large distances no longer provide us with an anschauliches Bild and we have to learn to live there without Anschauung (something like "without familiar everyday pictures"). In this case we realize that the antinomy of the infinitely small for the elementary particles is resolved in a very subtle way - in a way that neither Immanuel Kant nor the Greek philosophers could think of — the words "to divide" lose their sense.

If one wants to compare the insights of today's particle physics with any earlier philosophy, it could only be the philosophy of Plato, since the particles of today's physics are representations of symmetry groups - that's what quantum theory teaches us - and, therefore, the particles resemble the symmetric Platonic solids.

Perhaps one can mitigate the resignation to live without "Anschauung" in the following sense: We have to get rid of the "billiard ball particles" and to get used to a new kind of "Anschauung," oriented at symmetries and related concepts like operations, invariants, eigenvalues, etc. 
Today's standard theory of particles essentially assumes that all matter is made up of two families of particles, quarks and leptons, all of which are "pointlike" concentrations of quantum fields. In contrast to the composite "brick" and bound state picture, quantum structure suggests a "quantum ladder" with different energy (matter) levels where energy and mass related operations are implemented by eigenstates, e.g., by molecules, atoms, nuclei, hadrons, quarks, leptons. "Elementarity" is a question of the available energy. In this context also Dirac may be quoted from his Nobel lecture (1933):

The distinction between elementary particles and composite particles now becomes a matter of convenience. This reason alone is sufficient to compel one to give up the attractive philosophical idea that all matter is made up of one kind, or perhaps two kinds of bricks.

\section{Causality and Time}

The causal structure in physics is numerically parametrized by the naturally totally ordered reals $t \in \mathbb{R}$, used for the time translations. There is an operational buildup - by binary, quaternary, infinitely countable relations etc. - from the natural numbers over the integers, the rationals to the Cauchy-complete reals and the algebraically closed complex numbers $\mathbb{N} \subset \mathbb{Z} \subset \mathbb{Q} \subset \mathbb{R} \subset \mathbb{C}$ which is not repeated here. The algebraic closure of the complex numbers allows the diagonalization of normal operators with the determination of eigenvectors and eigenvalues which is of paramount importance to define "physical objects". With the canonical conjugation as field automorphism of the complex numbers, it is also possible to implement nontrivially the time reflection.

The additive reals are isomorphic as Lie group to the multiplicative dilations $\mathbb{R} \ni t \leftrightarrow$ $e^{t} \in \mathbf{D}(1)=\exp \mathbb{R}$. With Schur, the irreducible complex time representations are onedimensional $e^{t} \longrightarrow e^{\alpha t} \in \mathbf{G L}(\mathbb{C})=\mathbf{D}(1) \times \mathbf{U}(1)$, i.e., acting on a vector space $V \cong \mathbb{C}$ and characterized by a complex number $\alpha \in$ irrep $\mathbf{D}(1) \cong \mathbb{C}$, the unitary ones by an imaginary one $e^{t} \longmapsto e^{i \omega t}$ from the dual group $i \omega \in$ irrep $_{+} \mathbf{D}(1) \cong i \mathbb{R}$ with a real frequency (energy). Dual representations on a vector space $V \cong \mathbb{C}$ and its dual $V^{T} \cong \mathbb{C}$ (linear forms, $T$ for transposed) pair opposite frequencies and lead to time orbits:

$$
\begin{array}{lll}
e^{t} \longmapsto e^{i \omega t} \in \mathbf{U}(1), & \mathbf{D}(1) \times V \longrightarrow V, & e^{t} \bullet v=v(t)=e^{i \omega t} v, \\
e^{t} \longmapsto e^{-i \omega t} \in \mathbf{U}(1), & \mathbf{D}(1) \times V^{T} \longrightarrow V^{T}, & e^{t} \bullet \lambda=\lambda(t)=e^{-i \omega t} \lambda .
\end{array}
$$

Such representations arise for all translations - for time, Euclidean position (space) and Minkowskian spacetime - and characterize interaction free "objects". It starts with the harmonic oscillator with the creation and annihilation operators (the name is justified below) constituing a dual basis:

$$
\begin{aligned}
& V=\mathbb{C u}, V^{T}=\mathbb{C u}^{*}, \text { dual basis: }\left\langle\mathrm{u}^{*} \mathrm{u}\right\rangle=1, \\
& \text { time orbits: } \mathrm{u}(t)=e^{i \omega t} \mathrm{u}, \mathrm{u}^{*}(t)=e^{-i \omega t} \mathrm{u}^{*} .
\end{aligned}
$$

The selfdual direct sum space has real and imaginary combinations as basis which are used in physics as position and momentum with the time orbits in the two-dimensional selfdual representation:

$$
\begin{aligned}
\text { basis of } \mathbf{V}=V \oplus V^{T} \cong \mathbb{C}^{2}: & \mathbf{x}=\frac{\mathrm{u}+\mathrm{u}^{*}}{\sqrt{2}}, \mathbf{p}=i \frac{\mathrm{u}-\mathrm{u}^{*}}{\sqrt{2}} \\
& \left(\begin{array}{l}
\mathbf{x} \\
\mathbf{p}
\end{array}\right)(t)=\left(\begin{array}{cc}
\cos \omega t & \sin \omega t \\
-\sin \omega t & \cos \omega t
\end{array}\right)\left(\begin{array}{l}
\mathbf{x} \\
\mathbf{p}
\end{array}\right) .
\end{aligned}
$$


Since the one-dimensional case is so simple that one may overlook dimensional structures, it will by switched over to a possibly decomposable time representation on $V \cong \mathbb{C}^{s}$, $s=1,2, \ldots$, e.g., the isotropic three-dimensional harmonic oscillator for $s=3$, with bases:

$$
\begin{aligned}
& \text { basis of } \mathbf{V}=V \oplus V^{T} \cong \mathbb{C}^{2 s}:\left\{\begin{array}{l}
\mathbf{x}^{a}=\frac{\mathrm{u}^{a}+\mathrm{u}_{a}^{*}}{\sqrt{2}}, a=1, \ldots, s, \\
\mathbf{p}^{a}=i \frac{\mathrm{u}^{a}-\mathrm{u}_{a}^{*}}{\sqrt{2}},
\end{array}\right. \\
& \text { dual bases: }\left\{\begin{array} { l } 
{ \langle \mathrm { u } _ { a } ^ { * } , \mathrm { u } ^ { b } \rangle = \delta _ { a } ^ { b } , } \\
{ \langle \mathrm { u } ^ { a } , \mathrm { u } ^ { b } \rangle = 0 = \langle \mathrm { u } _ { a } ^ { * } , \mathrm { u } _ { b } ^ { * } \rangle , }
\end{array} \text { or } \left\{\begin{array}{l}
\left\langle i \mathbf{p}^{a}, \mathbf{x}^{b}\right\rangle=\delta^{a b}, \\
\left\langle\mathbf{x}^{a}, \mathbf{x}^{b}\right\rangle=0=\left\langle\mathbf{p}^{a}, \mathbf{p}^{b}\right\rangle .
\end{array}\right.\right.
\end{aligned}
$$

and time action:

$$
\begin{aligned}
& \begin{array}{l}
e^{t} \longmapsto e^{i \omega t} \mathbf{1}_{s} \in \mathbf{U}\left(\mathbf{1}_{s}\right), \\
e^{t} \longmapsto e^{-i \omega t} \mathbf{1}_{s} \in \mathbf{U}\left(\mathbf{1}_{s}\right),
\end{array} \text { time orbits: }\left\{\begin{array}{l}
\mathrm{u}^{a}(t)=e^{i \omega t} \mathrm{u}^{a}, \\
\mathrm{u}_{a}^{*}(t)=e^{-i \omega t} \mathrm{u}_{a}^{*},
\end{array}\right. \\
& \frac{d}{d t}\left(\begin{array}{c}
\mathbf{x}^{a} \\
\mathbf{p}^{a}
\end{array}\right)(t)=\left(\begin{array}{cc}
0 & \omega \\
-\omega & 0
\end{array}\right)\left(\begin{array}{l}
\mathbf{x}^{a} \\
\mathbf{p}^{a}
\end{array}\right), e^{t} \longmapsto \exp \left(\begin{array}{cc}
0 & \omega \\
-\omega & 0
\end{array}\right) t=\left(\begin{array}{cc}
\cos \omega t & \sin \omega t \\
-\sin \omega t & \cos \omega t
\end{array}\right) \in \mathbf{S O}(2) .
\end{aligned}
$$

The differential equations of motion may be derived classically by the usual formalisms with Lagrangian and Hamiltonian. The Hamitonian is the unit operator in the representation spaces with the familiar tensor product basis form:

$$
V \otimes V^{T} \oplus V^{T} \otimes V \ni \mathbf{H}=\omega \frac{\mathbf{1}_{s} \oplus \mathbf{1}_{s}^{T}}{2}=\omega \frac{\mathrm{u}_{a}^{*} \otimes \mathrm{u}^{a}+\mathrm{u}^{a} \otimes \mathrm{u}_{a}^{*}}{2}=\frac{\mathbf{x}^{a} \otimes \mathbf{x}^{a}+\mathbf{p}^{a} \otimes \mathbf{p}^{a}}{2} .
$$

\section{Quantum Structure and Hilbert Spaces}

Quantum theory relies on complex linear structures, so far illustrated by vector space duality $\left(V, V^{T}\right) \cong \mathbb{C}^{s}$, by direct sums $\mathbf{V}=V \oplus V^{T} \cong \mathbb{C}^{2 s}$ and tensor products $\left(V \otimes V^{T}, V^{T} \otimes\right.$ $V) \cong \mathbb{C}^{s^{2}}$ and $\mathbf{V} \otimes \mathbf{V} \cong \mathbb{C}^{4 s^{2}}$.

Quantum algebras for creation and annihilation operators $\mathrm{u} \stackrel{\star}{\leftrightarrow} \mathrm{u}^{\star}$ are characterized by (anti-)commutators, $[a, b]_{+}=\{a, b\}=a b+b a,[a, b]_{-}=[a, b]=a b-b a$ :

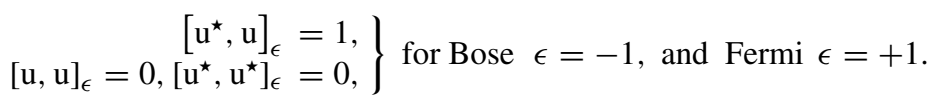

To obtain, in general, the quantum algebras $\mathbf{Q}_{\epsilon}\left(\mathbb{C}^{2 s}\right)$ for a complex finite-dimensional vector space $V \cong \mathbb{C}^{s}$ with dual space $V^{T}$ and dual bases $\left\langle\mathrm{u}_{a}^{\star} \mathrm{u}^{b}\right\rangle=\delta_{a}^{b}$, the tensor algebra $\otimes \mathbf{V}$ of the selfdual direct sum $\mathbf{V}=V \oplus V^{T} \cong \mathbb{C}^{2 s}$ is factorized by the minimal ideal which identifies the bilinear dual products (not the sesquilinear Hilbert space scalar products) and corresponding tensor (anti-)commutators:

$$
\mathbf{Q}_{\epsilon}\left(\mathbb{C}^{2 s}\right)=\bigotimes \mathbf{v} / \text { minimal ideal }\left(\begin{array}{l}
{\left[\mathrm{u}_{a}^{\star}, \mathrm{u}^{b}\right]_{\epsilon}-\left\langle\mathrm{u}_{a}^{\star} \mathrm{u}^{b}\right\rangle,} \\
{\left[\mathrm{u}^{a}, \mathrm{u}^{b}\right]_{\epsilon},\left[\mathrm{u}_{a}^{\star}, \mathrm{u}_{b}^{\star}\right]_{\epsilon}}
\end{array}\right) .
$$

The construction of the quantum algebras for a vector space is similar to the construction of the enveloping algebra for a Lie algebra with the identification of Lie bracket and corresponding tensor commutator. 
The complex $2^{2 s}$-dimensional Fermi quantum algebra and the complex $\aleph_{0}$-dimensional Bose quantum algebra are characterized by the duality-induced basic (anti-)commutators

$$
\begin{aligned}
& \text { in Fermi } \mathbf{Q}_{+}\left(\mathbb{C}^{2 s}\right) \cong \bigotimes^{s} \mathbf{Q}_{+}\left(\mathbb{C}^{2}\right):\left\{\begin{array}{l}
\left\{\mathrm{u}_{a}^{\star}, \mathrm{u}^{b}\right\}=\delta_{a}^{b}, \\
\left\{\mathrm{u}^{a}, \mathrm{u}^{b}\right\}=0,\left\{\mathrm{u}_{a}^{\star}, \mathrm{u}_{b}^{\star}\right\}=0,
\end{array}\right. \\
& \text { in Bose } \mathbf{Q}_{-}\left(\mathbb{C}^{2 s}\right) \cong \bigotimes^{s} \mathbf{Q}_{-}\left(\mathbb{C}^{2}\right):\left\{\begin{array}{l}
{\left[\mathrm{u}_{a}^{\star}, \mathrm{u}^{b}\right]=\delta_{a}^{b},} \\
{\left[\mathrm{u}^{a}, \mathrm{u}^{b}\right]=0,\left[\mathrm{u}_{a}^{\star}, \mathrm{u}_{b}^{\star}\right]=0 .}
\end{array}\right.
\end{aligned}
$$

Historically, they were used first for $s$ bosonic degrees of freedom of harmonic oscillators, e.g., the Bose case $\mathbf{Q}_{-}\left(\mathbb{C}^{3}\right)$ for 3-position $\mathbf{x}^{a}=\frac{\mathrm{u}^{a}+\mathrm{u}_{a}^{\star}}{\sqrt{2}}$ with momenta $\mathbf{p}^{a}=i \frac{\mathrm{u}^{a}-\mathrm{u}_{a}^{\star}}{\sqrt{2}}$ and $\left[i \mathbf{p}^{a}, \mathbf{x}^{b}\right]=\delta_{b}^{a}$, and angular momenta $\mathbf{J}^{a}=-i \epsilon^{a b c} \mathbf{x}^{b} \otimes \mathbf{p}^{c}$. Their structure is much more general: Quantum algebras $\mathbf{Q}_{\epsilon}\left(\mathbb{C}^{2 s}\right)$ give the quantization of $s$ fermionic or bosonic degrees of freedom.

The quantum algebra has representatives for the basic space endomorphisms $\mathbf{A L}(V) \ni$ $f=f_{a}^{b} \mathrm{u}^{a} \otimes \mathrm{u}_{b}^{\star}$ whose endomorphism commutator is in the same class (simply denoted by $=)$ as the quantum product commutator:

$$
\begin{aligned}
& f, g \in \mathbf{A} \mathbf{L}(V): \quad {[f, g]_{\circ}=f \circ g-g \circ f, } \\
& \text { in } \mathbf{Q}_{\epsilon}\left(\mathbb{C}^{2 s}\right): \quad[f, g]_{\otimes}=f \otimes g-g \otimes f, \quad[f, g]_{\circ}=[f, g]_{\otimes}, \\
& {\left[f, \mathrm{u}^{c}\right]=f_{a}^{c} \mathbf{u}^{a},\left[f, \mathbf{u}_{d}^{\star}\right]=-f_{d}^{b} \mathbf{u}_{b}^{\star} . }
\end{aligned}
$$

For instance in the quantum algebras $\mathbf{Q}_{\epsilon}\left(\mathbb{C}^{3}\right)$, the angular momenta for the infinitesimal rotations obey the familiar Lie brackets:

$$
\mathbf{J}^{a}=-\epsilon^{a b c} \mathrm{u}^{b} \otimes \mathrm{u}_{c}^{\star}: \mathbf{J}^{a} \circ \mathbf{J}^{b}-\mathbf{J}^{b} \circ \mathbf{J}^{b}=-\epsilon^{a b c} \mathbf{J}^{c}=\mathbf{J}^{a} \otimes \mathbf{J}^{b}-\mathbf{J}^{b} \otimes \mathbf{J}^{b}=\left[\mathbf{J}^{a}, \mathbf{J}^{b}\right] .
$$

Usually, the tensor quantum product sign $\otimes$ is omitted, e.g., $\mathbf{x}^{b} \mathbf{p}^{a}-\mathbf{p}^{a} \mathbf{x}^{b}=i \delta_{b}^{a}$ and $\mathbf{J}^{a}=-i \epsilon^{a b c} \mathbf{x}^{b} \mathbf{p}^{c}$. The class property, e.g., $\mathrm{u}=\mathrm{uu}^{\star} \mathrm{u}+\epsilon \mathrm{u}^{2} \mathrm{u}^{\star}$, is taken into account by "getting used to it" - similar as for the rationals, e.g., $\frac{3}{4}=\frac{9}{12}$.

The quantum algebras contain product representations of the basic space $V$ and its dual $V^{T}$. In such a way, quantum algebras are representation spaces of the basic space Lie algebra $\mathbf{A L}(V)$, e.g., the Bose quantum algebras with the time representations:

$$
\text { in } \begin{aligned}
\mathbf{Q}_{-}\left(\mathbb{C}^{2 s}\right): & \mathbf{H}=\omega \frac{\left\{\mathrm{u}_{a}^{*}, \mathrm{u}^{a}\right\}}{2}=\omega \frac{\overrightarrow{\mathbf{x}}^{2}+\overrightarrow{\mathbf{p}}^{2}}{2}, \\
& {\left[\mathbf{H}, \mathrm{u}^{a}\right]=\omega \mathrm{u}^{a},\left[\mathbf{H}, \mathrm{u}_{a}^{*}\right]=-\omega \mathrm{u}_{a}^{*}, } \\
& {[\mathbf{H}, \overrightarrow{\mathbf{x}}]=\omega \overrightarrow{\mathbf{p}},[\mathbf{H}, \overrightarrow{\mathbf{p}}]=-\omega \overrightarrow{\mathbf{x}}, } \\
& {\left[\mathbf{H}, \mathrm{u}_{a_{1}}^{*} \cdots \mathrm{u}_{a_{p}}^{*} \mathrm{u}^{a_{1}} \cdots \mathrm{u}^{a_{q}}\right]=(q-p) \omega \mathrm{u}_{a_{1}}^{*} \cdots \mathrm{u}_{a_{p}}^{*} \mathrm{u}^{a_{1}} \cdots \mathrm{u}^{a_{q}} . }
\end{aligned}
$$

Probabilities and probability amplitudes for quantum theories arise on complex Hilbert spaces. A conjugation $*$ of a vector space is an involutive antilinear isomorphism of dual spaces $V \ni v \stackrel{*}{\leftrightarrow} v^{*} \in V^{T}, v^{* *}=v$. Together with the linear dual product, it defines a nondegenerate sesquilinear form $V \times V \ni(v, w) \longmapsto\left\langle v^{*} w\right\rangle=\langle v \mid w\rangle \in \mathbb{C}$ which is characterized by a definite or indefinite invariance group $\mathbf{U}\left(n_{+}, n_{-}\right)$for $V \cong \mathbb{C}^{n_{+}+n_{-}}$. A definite Euclidean $\mathbf{U}(n)$-conjugation defines a scalar product and the structure of a Hilbert space. The geometry of complex Hilbert spaces is interpretable with quantum probability concepts (or vice versa) - vectors as probability amplitudes, scalar products with cosines as transition amplitudes and uncertainties, etc.

Complex Hilbert spaces for quantum theories have their origin in complex associative operator algebras $A$ with unit $1_{A} \in A$ and equipped with an antilinear reflection, $A \ni a \leftrightarrow$ 
$a^{\star}$, e.g., in quantum mechanics, a complex operator algebra with positions $\mathbf{x}=\mathbf{x}^{\star}$, momenta $\mathbf{p}=\mathbf{p}^{\star}$, angular momenta $\mathbf{J}=i \mathbf{L}=-\mathbf{J}^{\star}$, creation and annihilation operators $\mathrm{u} \leftrightarrow \mathrm{u}^{\star}$, etc. Bose and Fermi quantum algebras as important examples have been discussed above. Later on, convolution algebras with complex group functions will be used.

A linear continuous form $d \in A^{\prime} \subseteq A^{T}$ of the algebra $A$ is called positive, $d \succeq 0$, for positive values of the hermitian squares $a^{\star} a=\left(a^{\star} a\right)^{\star}$ :

$$
d: A \longrightarrow \mathbb{C}, d\left(a^{\star} a\right)=\|a\|_{d}^{2} \geq 0 \text { for all } a \in A .
$$

Algebra forms can be reflected $d \leftrightarrow d^{\star}$ with $d^{\star}(a)=\overline{d\left(a^{\star}\right)}$. They are symmetric for $d=d^{\star}$. A positive unital form, i.e., with $d\left(1_{A}\right)=1$, is called a state of the algebra.

The Cauchy-Schwarz inequality for a positive form is relevant for Heisenberg's uncertainty relation: It gives a lower bound for the prenorm product of two operators $a, b \in$ A:

$$
\begin{aligned}
\text { for all } \alpha \in \mathbb{R} \text { and } e^{i \beta} \in \mathbf{U}(1): \quad d\left(\left(a^{\star}+\alpha e^{-i \beta} b^{\star}\right)\left(a+\alpha e^{i \beta} b\right)\right) \geq 0 \\
\Rightarrow\|a\|_{d}^{2}\|b\|_{d}^{2} \geq\left[d\left(\frac{e^{i \beta} a^{\star} b+e^{-i \beta} b^{\star} a}{2}\right)\right]^{2},
\end{aligned}
$$

If the algebra elements are both symmetric or both antisymmetric, the product of their prenorms is bounded from below by the absolute $d$-value of a combination of their anticommutator and commutator:

$$
(a, b)= \pm\left(a^{\star}, b^{\star}\right) \Rightarrow\|a\|_{d}\|b\|_{d} \geq\left|d\left(\frac{\{a, b\}}{2} \cos \beta+\frac{[i a, b]}{2} \sin \beta\right)\right| .
$$

For special values of the phase, there remains only the commutator or only the anticommutator:

$$
\begin{aligned}
& \beta=0 \Rightarrow\|a\|_{d}\|b\|_{d} \geq\left|d\left(\frac{\{a, b\}}{2}\right)\right|, \\
& \beta=\frac{\pi}{2} \Rightarrow\|a\|_{d}\|b\|_{d} \geq\left|d\left(\frac{[i a, b]}{2}\right)\right| .
\end{aligned}
$$

With the standard deviation (square root of the variance) $\Delta a=\sqrt{\operatorname{var} a}$ of a symmetric algebra element in a state $d$,

$$
a=a^{\star}, a_{d}=a-d(a) 1_{A}=a_{d}^{\star} \Rightarrow\left\{\begin{array}{l}
d\left(a_{d}\right)=0, \\
(\Delta a)^{2}=d\left(a_{d}^{2}\right)=d\left(a^{2}\right)-d(a)^{2} \geq 0,
\end{array}\right.
$$

there arises for any pair of (anti)symmetric elements the uncertainty relation which bounds the product of their standard deviations from below by half the absolute state value of their commutator:

$$
(a, b)=\left(a^{\star}, b^{\star}\right) \Rightarrow \Delta a \Delta b=\left\|a_{d}\right\|\left\|b_{d}\right\| \geq \frac{1}{2}\left|d\left(\left[a_{d}, b_{d}\right]\right)\right|=\frac{1}{2}|d([a, b])| .
$$

The most famous example is Heisenberg's position-momentum uncertainty where the commutator involves the unit operator:

$$
[\mathbf{x}, \mathbf{p}]=\hbar i \mathbf{1}: \Delta \mathbf{x} \Delta \mathbf{p} \geq \frac{1}{2}|d([\mathbf{x}, \mathbf{p}])|=\frac{\hbar}{2} .
$$


Following the GNS-construction (Gelfand, Naimark, Segal), a positive form gives the algebra the structure of a pre-Hilbert space with prescalar product, prenorm, and topology (not necessarily Hausdorff):

$$
\begin{aligned}
\langle\|\rangle_{d}: A \times A \longrightarrow \mathbb{C},\langle a \| b\rangle_{d} & =d\left(a^{\star} b\right), \\
\|a\|_{d}^{2} & =\langle a \| a\rangle_{d}=d\left(a^{\star} a\right) \geq 0 .
\end{aligned}
$$

For a positive form, the $d$-trivial elements constitute the orthogonal $A_{d}^{\perp}$, a left ideal:

$$
d \succeq 0 \Rightarrow A_{d}^{\perp}=\left\{n \in A \mid d\left(n^{\star} n\right)=0\right\} .
$$

The classes $|A\rangle_{d}=A / A_{d}^{\perp}$ with respect to the orthogonal have a definite scalar product:

$$
\begin{gathered}
\langle\mid\rangle_{d}:|A\rangle_{d} \times|A\rangle_{d} \longrightarrow \mathbb{C},\langle a \mid b\rangle_{d}=d\left(a^{\star} b\right),\langle a \mid a\rangle_{d} \geq 0, \\
\langle n \mid n\rangle_{d}=0 \Longleftrightarrow n \in A_{d}^{\perp} \Longleftrightarrow|n\rangle_{d}=0 .
\end{gathered}
$$

The equivalence classes $>a_{d}=a+A_{d}^{\perp}$ are called state vectors. $d$-strictly positive operators $a \in A$ define nontrivial state vectors. The class $>1_{A d}$ of the algebra unit is a cyclic state vector, i.e., $\left.A\rangle 1_{A d}=\right\rangle A_{d}$. The state values can be written as expectation values of this cyclic state vector:

$$
d: A \longrightarrow \mathbb{C}, d(a)=\left\langle 1_{A}|a| 1_{A}\right\rangle_{d}
$$

The Cauchy completion of the scalar product space is the $d$-associate Hilbert space $A \stackrel{d}{\rightarrow}$ $A / A \stackrel{\perp}{\stackrel{\text { Cauchy }}{\longrightarrow}} \overline{|A\rangle_{d}}=V_{d}$. The algebra is represented on $|A\rangle_{d}$, dense in $V_{d}$. Pure states (no nontrivial positive combination by two states) lead to irreducible algebra representations.

The Fock state $\langle\ldots\rangle$ of the Bose and Fermi quantum algebras above is defined by nontrivial values for the abelian subalgebra with the $\mathbf{U}(1)$-invariants $\left\{\left(u^{\star} u\right)^{k} \mid k=\right.$ $0,1,2, \ldots\}$ :

$$
\begin{aligned}
\mathbf{Q}_{\epsilon}\left(\mathbb{C}^{2}\right) \longrightarrow \mathbb{C}, & \left\langle\left(\mathrm{u}^{\star} \mathrm{u}\right)^{k}\right\rangle=1, k=0,1,2, \ldots, \\
& \left\langle\left[\mathrm{u}^{\star} \mathrm{u}, a\right]\right\rangle=0, a \in \mathbf{Q}_{\epsilon}\left(\mathbb{C}^{2}\right) .
\end{aligned}
$$

It is a unital form, $\langle 1\rangle=1$, and positive:

$$
\begin{aligned}
\Rightarrow \quad\left\langle a \mathrm{u}^{\star}\right\rangle & =0,\left\langle\left(\mathrm{u}^{\star}\right)^{k}(\mathrm{u})^{l}\right\rangle=\delta^{k l} k !,\left\langle a^{\star} a\right\rangle \geq 0, \\
\text { e.g., } & \left\langle\mathrm{u}^{\star} \mathrm{u}\right\rangle=\|\mathrm{u}\|^{2}=1 \text { for creation operator, } \\
\left\langle\mathrm{uu}^{\star}\right\rangle & =\left\|\mathrm{u}^{\star}\right\|^{2}=0 \text { for annihilation operator. }
\end{aligned}
$$

The quantum algebras are no $\mathrm{C}^{*}$-algebras where $\|a\|=\| a^{\star}||$ for all elements.

The orthogonals for the quantum algebras above are given by the left ideal $\mathbf{Q}_{\epsilon}\left(\mathbb{C}^{2}\right) \mathrm{u}^{\star}$ with the annihilation operator. The classes $\mathbf{Q}_{\epsilon}\left(\mathbb{C}^{2}\right) / \mathbf{Q}_{\epsilon}\left(\mathbb{C}^{2}\right) \mathrm{u}^{\star}$ with the Fock state vectors can be spanned by the monomials $\left\{(\mathrm{u})^{k} \mid k=0,1,2, \ldots\right\}$ of the creation operator, for Fermi by two elements $\{1, \mathrm{u}\}$. Usually one uses the occupation number notation for the normalized $k$-quantum state vectors:

$$
\begin{aligned}
& |k\rangle=\frac{1}{\sqrt{k !}}(u)^{k}+\mathbf{Q}_{\epsilon}\left(\mathbb{C}^{2}\right) u^{\star}, k= \begin{cases}0,1,2, & \text { for Fermi } \epsilon=+1, \\
0,1,2, \ldots, & \text { for Bose } \epsilon=-1,\end{cases} \\
& \langle l \mid k\rangle=\delta^{l k},\langle l u\rangle k=\delta_{l}^{k+1} \sqrt{k+1}=\left\langle k \mathrm{u}^{\star}\right\rangle l .
\end{aligned}
$$

The class of the algebra unit $|0\rangle=1+\mathbf{Q}_{\epsilon}\left(\mathbb{C}^{2}\right) \mathrm{u}^{\star}$ is the zero-quantum state vector, used as ground-state of the harmonic oscillators. The annihilation ideal in the definition of the ground-state $|0\rangle$ may remind of Dirac's attempts to postulate a populated ground-state (Dirac sea). The Fermi-Fock Hilbert space $V_{+}^{\text {Fock }} \cong \mathbb{C}^{2}$ has basis $\{|0\rangle,|1\rangle\}$, the Bose-Fock 
Hilbert space with Hilbert basis $\{|k\rangle \mid k=0,1,2, \ldots\}$ is isomorphic to the square integrable position functions $V_{-}^{\text {Fock }} \cong L^{2}(\mathbb{R})$.

With the "vacuum" $|0\rangle$, all occupation number vectors:

$$
|k\rangle=\frac{(\mathrm{u})^{k}}{\sqrt{k !}}|0\rangle, k=0,1,2, \ldots,
$$

define quantum algebra states $b \longmapsto\langle k|b|\rangle k=\frac{1}{k !}\left\langle\left(\mathrm{u}^{\star}\right)^{k} b(\mathrm{u})^{k}\right\rangle$. The Fock state values are the "vacuum" expectation values $\langle b\rangle=\langle 0|b| 0\rangle$.

The Fermi and Bose Fock-Hilbert spaces are used for the representations of time, position and spacetime translations, especially for free particles. In the massive particle case, the creation and annihilation operators are distributions of the energy-momentum hyperboloid $\vec{q} \in \mathcal{Y}^{3}$ with $q_{0}^{2}-\vec{q}^{2}=m^{2}$ (the discrete dimension $\mathrm{u}^{a} \in V \cong \mathbb{C}^{s}$ is replaced by a "continuous" one $\left.\mathrm{u}(\vec{q}) \in V \cong \mathbb{C}^{\mathcal{Y}^{3}}\right)$, possibly with additional discrete indices for the eigenvalues of compact homogeneous groups ((iso)spin, color etc.):

$$
\begin{aligned}
{\left[\mathrm{u}_{\alpha}^{\star}(\vec{p}), \mathrm{u}^{\beta}(\vec{q})\right]_{\epsilon} } & =2 q_{0} \delta\left(\frac{\vec{q}-\vec{p}}{2 \pi}\right) \delta_{\alpha}^{\beta}, \text { with } q_{0}=\sqrt{\vec{q}^{2}+m^{2}} \\
\left\langle 0\left|\mathrm{u}_{\alpha}^{\star}(\vec{p}) \mathrm{u}^{\beta}(\vec{q})\right| 0\right\rangle & =\left\langle m^{2} ; \vec{p}, \alpha \mid m^{2} ; \vec{q}, \beta\right\rangle=2 q_{0} \delta\left(\frac{\vec{q}-\vec{p}}{2 \pi}\right) \delta_{\alpha}^{\beta}
\end{aligned}
$$

distributive Hilbert basis: $\left\{\left|m^{2} ; \vec{q}, \alpha\right\rangle=\mathrm{u}^{\alpha}(\vec{q})|0\rangle \mid \vec{q} \in \mathbb{R}^{3}, \alpha=1, \ldots, d.\right\}$.

Product states use appropriately (anti)symmetrized tensor product distributions, e.g.,

$$
\left|\vec{q}_{1}, \ldots, \vec{q}_{k}, \alpha_{1}, \ldots, \alpha_{k}\right\rangle \sim \mathrm{u}^{\alpha_{1}}\left(\vec{q}_{1}\right) \otimes \cdots \otimes \mathrm{u}^{\alpha_{k}}\left(\vec{q}_{k}\right)|0\rangle
$$

Lie algebra elements have to integrate the momentum operators on the mass hyperboloid, e.g., for the rotation (spin) Lie algebra:

$$
\begin{aligned}
& \underline{\mathbf{O}}=\oplus \boldsymbol{\int} \frac{d^{3} q}{(2 \pi)^{3} 2 q_{0}} \mathrm{u}^{b}(\vec{q}) \mathbf{O}_{b}^{c} \mathrm{u}_{c}^{\star}(\vec{q}),\left[\underline{\mathbf{O}}_{1}, \underline{\mathbf{O}}_{2}\right]=\left[\mathbf{O}_{1}, \mathbf{O}_{2}\right], \\
& \mathbf{J}^{a}=\oplus \boldsymbol{\int} \frac{d^{3} q}{(2 \pi)^{3} 2 q_{0}} \mathbf{J}^{a}(\vec{q}), \mathbf{J}^{a}(\vec{q})=-\epsilon^{a b c} \mathbf{u}^{b}(\vec{q}) \otimes \mathrm{u}_{c}^{\star}(\vec{q}), \\
& {\left[\mathbf{J}^{a}(\vec{p}), \mathbf{J}^{b}(\vec{q})\right]=-2 q_{0} \delta\left(\frac{\vec{q}-\vec{p}}{2 \pi}\right) \epsilon^{a b c} \mathbf{J}^{c}(\vec{q}),\left[\mathbf{J}^{a}, \mathbf{J}^{b}\right]=-\epsilon^{a b c} \mathbf{J}^{c} .}
\end{aligned}
$$

The momentum integrals become, in a formulation with spacetime fields, position integrals over currents $\left(\mathbf{J}_{k}^{a}(\vec{x})\right)_{k=0,1,2,3}$, e.g., $\mathbf{J}^{a}=\int d^{3} x \mathbf{J}_{0}^{a}(\vec{x})$.

\section{The Heisenberg Lie Algebra and Group}

It is possible to relate the quantum-mechanical Born-Heisenberg commutation relations to a Lie algebra structure: In nonrelativistic quantum mechanics the position-momentum operators $(\overrightarrow{\mathbf{x}}, \overrightarrow{\boldsymbol{p}})$ come with the nontrivial commutator $\mathbf{I}$ - think of the Heisenberg operator I as an abstract vector, not as the imaginary unit $i !\{\overrightarrow{\mathbf{x}}, \overrightarrow{\boldsymbol{p}}, \mathbf{I}\}$ span as basis the power three nilpotent real Heisenberg Lie algebra $\log \mathbf{H}(s) \cong \mathbb{R}^{1+2 s}$, characterized by $s=1,2, \ldots$, nontrivial brackets for $\overrightarrow{\mathbf{x}}=\left(\mathbf{x}^{1}, \ldots, \mathbf{x}^{s}\right)$ and $\overrightarrow{\mathbf{p}}$ :

$$
[\overrightarrow{\mathbf{x}}, \overrightarrow{\mathbf{p}}]=\mathbf{1}_{s} \mathbf{I},[\overrightarrow{\mathbf{x}}, \mathbf{I}]=0=[\overrightarrow{\mathbf{p}}, \mathbf{I}]
$$

The Lie algebra of a group is denoted with the $\log$ arithm $L=\log G$, the exponent denotes the universal covering group $\exp L=\bar{G}$. 
The Heisenberg Lie algebra has a faithful nonunitary real $(2+s)$-dimensional representation where the Heisenberg operator $\mathbf{I}$ is not proportional to the unit matrix:

$$
\begin{aligned}
& \log \mathbf{H}(s) \quad \ni \vec{\pi} \overrightarrow{\mathbf{x}}+\vec{\xi} \overrightarrow{\mathbf{p}}+\tau \mathbf{I} \longmapsto\left(\begin{array}{cc|c}
0 & \vec{\pi} & \tau \\
0 & \mathbf{0}_{s} & \vec{\xi} \\
\hline 0 & 0 & 0
\end{array}\right) \in \mathbf{A L}(2+s, \mathbb{R}), \\
& \log \mathbf{H}(s)^{2} \ni\left[\vec{\pi}_{1} \overrightarrow{\mathbf{x}}+\vec{\xi}_{1} \overrightarrow{\mathbf{p}}+\tau_{1} \mathbf{I}, \vec{\pi}_{2} \overrightarrow{\mathbf{x}}+\vec{\xi}_{2} \overrightarrow{\mathbf{p}}+\tau_{2} \mathbf{I}\right]=\left(\vec{\pi}_{1} \vec{\xi}_{2}-\vec{\xi}_{1} \vec{\pi}_{2}\right) \mathbf{I}, \\
& \log \mathbf{H}(s)^{3}=\{0\} .
\end{aligned}
$$

All operators - positions, momenta and the Heisenberg operator — are representable by nilpotent matrices, e.g., for $s=1$ :

$$
\begin{aligned}
& \mathbf{x} \longmapsto\left(\begin{array}{lll}
0 & 1 & 0 \\
0 & 0 & 0 \\
0 & 0 & 0
\end{array}\right), \mathbf{p} \longmapsto\left(\begin{array}{lll}
0 & 0 & 0 \\
0 & 0 & 1 \\
0 & 0 & 0
\end{array}\right), \mathbf{I} \longmapsto\left(\begin{array}{lll}
0 & 0 & 1 \\
0 & 0 & 0 \\
0 & 0 & 0
\end{array}\right), \\
& \mathbf{x} \circ \mathbf{x}=0, \mathbf{p} \circ \mathbf{p}=0, \mathbf{I} \circ \mathbf{I}=0,[\mathbf{x}, \mathbf{p}]=\mathbf{I},[\mathbf{x}, \mathbf{I}]=0=[\mathbf{p}, \mathbf{I}] .
\end{aligned}
$$

Exponentiation of the Heisenberg Lie algebra gives the noncompact real $(1+2 s)$ dimensional Heisenberg group $\mathbf{H}(s)$, for illustration again in a nonunitary faithful real $(2+s)$-dimensional representation:

$$
\begin{aligned}
\mathbf{H}(s) & \longrightarrow \mathbf{G L}(2+s, \mathbb{R}), \\
\exp (\vec{\pi} \overrightarrow{\mathbf{x}}+\vec{\xi} \overrightarrow{\mathbf{p}}+\tau \mathbf{I}) & \longmapsto \exp \left(\begin{array}{cc|c}
0 & \vec{\pi} & \tau \\
0 & 0 & \vec{\xi} \\
\hline 0 & 0 & 0
\end{array}\right)=\left(\begin{array}{cc|c}
1 & \vec{\pi} & \tau+\vec{\pi} \vec{\xi} \\
0 & 1_{s} & \vec{\xi} \\
\hline 0 & 0 & 1
\end{array}\right), \\
e^{\vec{\pi}_{1} \overrightarrow{\mathbf{x}}+\vec{\xi}_{1} \overrightarrow{\mathbf{p}}+\tau_{1} \mathbf{I}} \circ e^{\vec{\pi}_{2} \overrightarrow{\mathbf{x}}+\vec{\xi}_{2} \overrightarrow{\mathbf{p}}+\tau_{2} \mathbf{I}} & =e^{\left(\vec{\pi}_{1}+\vec{\pi}_{2}\right) \overrightarrow{\mathbf{x}}+\left(\vec{\xi}_{1}+\vec{\xi}_{2}\right) \overrightarrow{\mathbf{p}}+\left(\tau_{1}+\tau_{2}-\vec{\pi}_{2} \vec{\xi}_{1}\right) \mathbf{I}} .
\end{aligned}
$$

It is a semidirect product with semidirect Lie algebra with the position translations as homogeneous contribution $e^{\mathbb{R}^{s}}$ acting on $(1+s)$-dimensional translations spanned by the Heisenberg operator I and the momenta $\overrightarrow{\mathbf{p}}$ :

$$
\begin{aligned}
\log \mathbf{H}(s) \cong & \mathbb{R}^{s} \vec{\oplus} \mathbb{R}^{1+s} \\
\mathbf{H}(s) \cong & e^{\mathbb{R}^{s} \overrightarrow{\times}} \mathbb{R}^{1+s} \ni e^{\vec{\pi} \overrightarrow{\mathbf{x}}+\vec{\xi} \overrightarrow{\mathbf{p}}+\tau \mathbf{I}}=e^{\vec{\xi} \overrightarrow{\mathbf{p}}+\tau \mathbf{I}} \emptyset e^{\vec{\pi} \overrightarrow{\mathbf{x}}} \\
& e^{\mathbb{R}^{s}} \times \mathbb{R}^{1+s} \longrightarrow \mathbb{R}^{1+s},\left(\begin{array}{ll}
1 & \vec{\pi} \\
0 & \mathbf{1}_{s}
\end{array}\right)\left(\begin{array}{c}
\tau \\
\vec{\xi}
\end{array}\right)=\left(\begin{array}{c}
\tau+\vec{\pi} \vec{\xi} \\
\vec{\xi}
\end{array}\right) .
\end{aligned}
$$

The familiar Hilbert space with the square-integrable function classes $L^{2}\left(\mathbb{R}^{s}\right)$ of position translations $\vec{x} \in \mathbb{R}^{s}, s=1,2, \ldots$, as eigenvalues of $\overrightarrow{\mathbf{x}}$ and the momentum representation $\overrightarrow{\mathbf{p}} \longmapsto-i \hbar \vec{\partial}$ is defined by the faithful unitary representations. In the complex infinitedimensional Hilbert representations of the Heisenberg group, the invariant Heisenberg operator $\mathbf{I} \longmapsto i \hbar \mathbf{1}$ is represented by the unit multiplied with one nontrivial spectral value $i \hbar \in \mathbf{s p e c} \mathbf{I}=i \mathbb{R}$ as action unit (Planck's constant). With the trace property of $[\mathbf{x}, \mathbf{p}]=\mathbf{I}$ there is no finite-dimensional representation with the Heisenberg operator as unit $\mathbf{I} \longmapsto i \mathbf{1}_{n}$.

For a quantum Hamiltonian $\mathbf{H}=\frac{\mathbf{p}^{2}}{2}+V(\mathbf{x})$, the mass point equations of motion $\frac{d \mathbf{x}}{d t}=$ $\frac{\partial \mathbf{H}}{\partial \mathbf{p}}=\mathbf{p}$ and $\frac{d \mathbf{p}}{d t}=-\frac{\partial \mathbf{H}}{\partial \mathbf{x}}=-\frac{d V}{d \mathbf{x}}$ for phase space time orbits $t \longmapsto(\mathbf{x}(t), \mathbf{p}(t))$ are inappropriate. In nonrelativistic wave mechanics, one looks for time translation eigenvectors, given by position-dependent Schrödinger functions:

$$
\begin{gathered}
\psi_{E}(t)=e^{i E t}|E\rangle: \frac{d}{d t} \boldsymbol{\psi}_{E}(t)=i \mathbf{H} \psi_{E}(t)=i E \boldsymbol{\psi}_{E}(t), \\
|E\rangle \cong \psi_{E} \in L^{2}\left(\mathbb{R}^{3}\right): \mathbf{H}|E\rangle \cong\left(-\frac{1}{2} \vec{\partial}^{2}+V(\vec{x})\right) \psi_{E}(\vec{x})=E \psi_{E}(\vec{x}) .
\end{gathered}
$$

The Schrödinger functions are coefficients of position representations. They lead to position measures $d^{3} x|\psi(\vec{x})|^{2}$ for probabilities of operators from position motion groups like translations, rotations for spheres, or Lorentz transformations for hyperboloids. 


\section{Operational Positions}

What characterizes objects and particles "in" time and space; what determines the "quantum ladder" for (binding) energies and masses and their characteristic units? In the operational framework, quantum theory of time, position and spacetime is determined by representations of the time-, position- and spacetime-defining operation groups. Free particles and interactions implement time and position operations, abelian and nonabelian ones, respectively.

For quantum mechanics "in" - better "of" or "for" 3-position, the Hilbert space $L^{2}\left(\mathbb{R}^{3}\right)$ with the square integrable position coefficients is of paramount importance. The $\mathbb{R}^{3}$-position may be a parametrization of different operational positions, e.g., of the abelian translation group, or for the position operator of the nonabelian Heisenberg Lie group with Lie algebra $\left[\mathbf{x}^{a}, \mathbf{p}^{b}\right]=\delta^{a b} i \mathbf{1}$, or of a coset manifold with classes of an operation group, interpreted as position, e.g., of hyperbolic position $\mathcal{Y}^{3} \cong \mathbb{R}^{3}$. In the Schrödinger picture of nonrelativistic quantum mechanics, the energy as time invariant is expressed by position representation eigenvalues and invariants - for the Hamiltonian $-i \frac{d}{d t} \cong \mathbf{H}=\frac{\overrightarrow{\mathbf{p}}^{2}}{2}+V(\overrightarrow{\mathbf{x}}) \cong-\frac{1}{2} \vec{\partial}^{2}+V(\vec{x})$.

Free objects and particles represent abelian translations, i.e., flat spaces, with the invariant $\mathbf{H}=\frac{\overrightarrow{\mathbf{p}}^{2}}{2}$. Composite states and interactions represent nonabelian operations, which, for basic structures, come as motion groups of curved position manifolds as determined by the potential $V(\overrightarrow{\mathbf{x}})$, e.g., as rotation groups $\mathbf{S O}(1+s)$ for the spheres $\Omega^{s}$ or as Lorentz groups $\mathbf{S O}_{0}(1, s)$ for the hyperboloids $\mathcal{Y}^{s}$, both with $s \geq 2$. In general relativity, the potential $V$ is related to the metrical tensor $\mathbf{g}$ and the curvature $\mathbf{R}$ of the underlying manifold, here of the position.

"Flat" and "curved" can be put in correspondence with "free" and "interacting" and, also, with time and position (space), as given in the following table to indicate a coarse parallelism of concepts:

\begin{tabular}{|c|c|c|c|c|c|}
\hline & Manifold & Group & Diff. eq. & Dynamics & Fields \\
\hline Time & one-dimensional & abelian & linear & free & $\begin{array}{l}\text { free leptons } \\
\text { (on shell) }\end{array}$ \\
\hline Position & curved & nonabelian & nonlinear & interacting & $\begin{array}{l}\text { confined quarks } \\
\text { (off shell) }\end{array}$ \\
\hline
\end{tabular}

The physically most important problem of nonrelativistic quantum theory is the atomic spectrum with its energies related to the eigenvalues of the Hamiltonian with the KeplerCoulomb potential. Its nature-realized structure tells us about the position model represented by the Schrödinger wave functions. As first elaborated by Pauli and Fock, the Lenz-Runge invariance (classical perihel conservation) of the Hamiltonian for the time translations involves a noncompact-compact pair of position operation groups with the Lorentz group $\mathbf{S O}_{0}(1,3)$ and the rotation group $\mathbf{S O}(4)$ as motion groups for their corresponding rotation group classes, i.e., for the 3 -hyperboloid $\mathcal{Y}^{3}$ and the 3 -sphere $\Omega^{3}$, respectively. It will be argued ahead that the wave functions of the nonrelativistic hydrogen atom are coefficients of Hilbert representations of hyperbolic position $\mathcal{Y}^{3} \cong \mathbf{S O}_{0}(1,3) / \mathbf{S O}(3)$. 
The maximally symmetric three-dimensional operational positions with definite metric parametrize rotation group classes. They are given by compact spherical, and noncompact flat and hyperbolic manifolds:

$$
\begin{array}{lll}
\Omega^{3} \cong \mathbf{S O}(4) / \mathbf{S O}(3) & \cong[\mathbf{S U}(2) \times \mathbf{S U}(2)] / \mathbf{S U}(2), & k=i^{2}=-1, \\
\mathbb{E}^{3} \cong \mathbf{S O}(3) \overrightarrow{\times} \mathbb{R}^{3} / \mathbf{S O}(3) \cong \mathbf{S U}(2) \times \mathbb{R}^{3} / \mathbf{S U}(2), & k=0^{2}=0, \\
\mathcal{Y}^{3} \cong \mathbf{S O}_{0}(1,3) / \mathbf{S O}(3) \cong \mathbf{S L}(2, \mathbb{C}) / \mathbf{S U}(2), & k=1^{2}=1,
\end{array}
$$

$$
\begin{aligned}
& \text { with metric } d s_{k}^{2}=\frac{d \vec{x}^{2}}{\left(1-k \frac{\vec{x}^{2}}{4}\right)^{2}}=\left(\begin{array}{c}
d \theta^{2} \\
d r^{2} \\
d \psi^{2}
\end{array}\right)+\left(\begin{array}{c}
\sin ^{2} \theta \\
r^{2} \\
\sinh ^{2} \psi
\end{array}\right) d \omega_{2}^{2}, \\
& \Omega^{2} \cong \mathbf{S O}(3) / \mathbf{S O}(2) \text { with metric } d \omega_{2}^{2}=d \Theta^{2}+\sin ^{2} \Theta d \phi^{2} \text {. }
\end{aligned}
$$

The positions $\mathbb{M} \in\left\{\Omega^{3}, \mathbb{E}^{3}, \mathcal{Y}^{3}\right\}$ can be taken as bases of coset bundles $\mathbf{S O}(3)(\mathbb{M})$ with $\mathbf{S O}(3) \sim \mathbf{S U}(2)$ as typical fiber.

The conformally Euclidean compact sphere $\Omega^{3}$ with Killing-form definiteness $k=-1$ — with invariant $P^{2}>0$ for metric $\frac{d \vec{x}^{2}}{\left(1+\frac{P^{2} \vec{x}^{2}}{4}\right)^{2}}-$ may be called Einstein position since it was used in the first attempt of Einstein for a cosmological model. The two noncompact partners are flat Euclidean position $\mathbb{E}^{3}$ with $k=0$ and conformally Euclidean hyperbolic Kepler position $\mathcal{Y}^{3}$ with $k=1$ or invariant $(i B)^{2}<0$ for metric $\frac{d \vec{x}^{2}}{\left(1-\frac{B^{2} \vec{x}^{2}}{4}\right)^{2}}$. Euclidean position can be considered as "flattened," i.e., as Inönü-Wigner contracted curved position, where, for hyperbolic position, the boosts are contracted to the position translations. This is mathematically isomorphic to the original Inönü-Wigner expansion of the Galilei velocity transformations into the Lorentz group boosts $\mathbf{S O}(3) \overrightarrow{\times} \mathbb{R}^{3} \stackrel{0 \longleftarrow \frac{1}{c}}{\longleftarrow} \mathbf{S O}_{0}(1,3)$ with the speed of light as the contraction-expansion parameter.

Without time and position connecting transformations, one may define spherical, flat and hyperbolic non-relativistic space-times $\mathbb{R} \times \mathbb{M}$ with the Einstein, Euclid and Kepler position, respectively, as direct factor for time $\mathbb{R}$.

With the introduction of half-integer spin structures in complex representation spaces, the real rotation and Lorentz groups can be replaced by their twofold cover groups. Therefore, the maximally symmetric three-dimensional positions can be formulated as classes of the unitary group $\mathbf{S U}(2) \sim \mathbf{S O}(3)$. The isotropy group for the 3 -sphere $\Omega^{3}$ is the "diagonal" group $(u, u) \in \mathbf{S U}(2) \times \mathbf{S U}(2)$. The Lorentz group $\mathbf{S L}(2, \mathbb{C}) \sim \mathbf{S O}_{0}(1,3)$ is familiar as a special relativistic spacetime group, i.e., acting on the spacetime tangent translations $\mathbb{R}^{4}$. As the motion group of hyperbolic position $\mathcal{Y}^{3}$, it does not affect time. The sphere and the hyperboloid and their motion groups constitute compact-noncompact pairs as defined by the imaginary-real transition $(i \mathbb{R})^{3} \leftrightarrow \mathbb{R}^{3}$, which relates their global rank two Lie algebras with the rotation Lie algebra $\log \mathbf{S U}(2) \cong(i \mathbb{R})^{3}$ :

$$
\begin{aligned}
\log [\mathbf{S U}(2) \times \mathbf{S U}(2)] & \cong(i \mathbb{R})^{3} \oplus(i \mathbb{R})^{3} \\
& \Rightarrow \log [\mathbf{S U}(2) \times \mathbf{S U}(2)] / \log \mathbf{S} \mathbf{U}(2) \cong(i \mathbb{R})^{3}, \\
\log \mathbf{S L}(2, \mathbb{C}) & \cong(i \mathbb{R})^{3} \oplus \mathbb{R}^{3} \\
& \Rightarrow \log \mathbf{S L}(2, \mathbb{C}) / \log \mathbf{S U}(2) \cong \mathbb{R}^{3}
\end{aligned}
$$


The Lie algebras of the three real six-dimensional motion groups have two independent invariants - always a discrete one (for compact Cartan subgroup $\mathbf{S O}(2)$ ) and, in addition, a discrete or a continuous (for noncompact Cartan subgroup $\mathbf{S O}_{0}(1,1) \cong \mathbb{R}$ ) one, explicitly in bases and with structure constants:

$$
\begin{aligned}
& \log \mathbf{S O}(4), k=-1:\left\{\begin{array}{c}
{\left[\mathbf{J}^{a}, \mathbf{J}^{b}\right]=-\epsilon^{a b c} \mathbf{J}^{c},} \\
{\left[\mathbf{J}^{a}, \mathbf{K}^{b}\right]=-\epsilon^{a b c} \mathbf{K}^{c},\left[\mathbf{K}^{a}, \mathbf{K}^{b}\right]=-\epsilon^{a b c} \mathbf{J}^{c},} \\
\text { invariants: }-\overrightarrow{\mathbf{J}}^{2}-\overrightarrow{\mathbf{K}}^{2},
\end{array} \overrightarrow{\mathbf{J} \mathbf{K},},\right. \\
& \text { noncompact } \leftrightarrow \text { compact } \uparrow \overrightarrow{\mathbf{B}} \leftrightarrow i \overrightarrow{\mathbf{K}}
\end{aligned}
$$

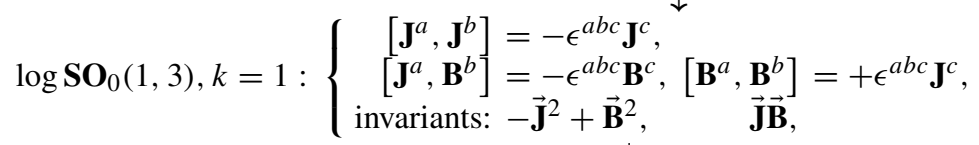

$$
\begin{aligned}
& \text { contraction: curved } \stackrel{\leftrightarrow \rightarrow 0}{\rightarrow} \text { flat }\rfloor \text { with } \overrightarrow{\mathbf{B}}=\frac{1}{x} \iota \overrightarrow{\mathbf{p}}
\end{aligned}
$$

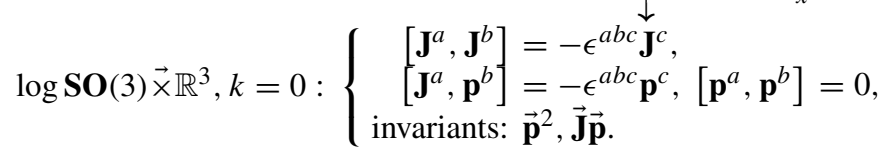

The groups $\mathbf{S O}(4)$ and $\mathbf{S O}_{0}(1,3)$ have a compact-noncompact pair of symmetric orthogonal Lie algebras. The flat structures arise by contraction from the curved ones.

The tangent Euclidean group $\mathbf{S O}(3) \overrightarrow{\times} \mathbb{R}^{3}$, common to all three maximally symmetric positions above, is implemented by free scattering states; nonflat hyperbolic position and its motion group $\mathbf{S L}(2, \mathbb{C}) \sim \mathbf{S O}_{0}(1,3)$ is implemented by the nonrelativistic atomic spectrum (more ahead).

\section{Special Position Functions}

The representation coefficients and distributions of maximally symmetric operational positions with odd dimensions $s=1,3, \ldots$, use Macdonald, Bessel and Neumann functions with half-integer indices. Characteristic $\mathbf{O}(3)$-invariant special functions come with a positive (spherical), a trivial (flat), or a negative (hyperbolic) invariant as poles $\vec{q}^{2}=$ $\left(P^{2}, 0,-B^{2}\right)$ for definiteness $k=\iota^{2}=(-1,0,1)$ in the complex $|\vec{q}|$-plane with the compact-nocompact relation $i|P| \leftrightarrow|B|$ :

$$
\left.\begin{array}{rl}
\int \frac{d^{3} q}{2 \pi^{2}} \frac{1}{\vec{q}^{2}+i o-P^{2}} e^{i \vec{q} \vec{x}} & =\frac{e^{-i|P| r}}{r}, \\
\int \frac{d^{3} q}{2 \pi^{2}} \frac{1}{\vec{q}^{2}} e^{i \vec{q} \vec{x}} & =\frac{1}{r}, \\
\int \frac{d^{3} q}{2 \pi^{2}} \frac{1}{\vec{q}^{2}+B^{2}} e^{i \vec{q} \vec{x}} & =\frac{e^{-|B| r}}{r},
\end{array}\right\} \begin{aligned}
& \int \frac{d^{3} q}{2 \pi^{2}} \frac{1}{\iota=(i, 0,1) .} e^{i \vec{q} \vec{x}}=\frac{e^{-i r}}{r}, \\
& \iota=(i o+1)
\end{aligned}
$$

The simple pole distribution with positive momentum invariant $\vec{q}^{2}=P^{2}>0$ contains contributions for two different groups via the real-imaginary decomposition $\pm \frac{1}{i \pi} \frac{1}{a \mp i o}=$ $\delta(a) \pm \frac{1}{i \pi} \frac{1}{a_{\mathrm{P}}}$ for $a \in \mathbb{R}$ into positive Dirac $\delta$-distribution and principal value $\mathrm{P}$ contribution:

$$
\text { for } \begin{aligned}
\mathbb{E}^{3}: \int \frac{d^{3} q}{2 \pi} \delta\left(\vec{q}^{2}-P^{2}\right) e^{i \vec{q} \vec{x}} & =\frac{\sin |P| r}{r}, \\
\int \frac{d^{3} q}{2 \pi^{2}} \frac{1}{\vec{q}_{\mathrm{P}}^{2}-P^{2}} e^{i \vec{q} \vec{x}} & =\frac{\cos P r}{r} .
\end{aligned}
$$


The Dirac distribution gives an $(r=0)$-regular spherical Bessel function as scalar translation representation coefficient of the Euclidean group $\mathbf{S O}(3) \overrightarrow{\times} \mathbb{R}^{3}$ for a free scattering state with the momentum $P^{2}$ as translation invariant:

$$
\text { for } \mathbb{E}^{3}:\left(-\vec{\partial}^{2}-P^{2}\right) \frac{\sin |P| r}{r}=0 .
$$

The principal value contribution (spherical Neumann function) and the Kepler and Yukawa potentials (hyperbolic Macdonald functions) are representation distributions. The kernels $\left(-\vec{\partial}^{2}+k\right)^{-1}$ are used for interactions. They have an $(r=0)$-singularity and obey inhomogeneous Laplace equations:

$$
\begin{aligned}
\left(-\vec{\partial}^{2}-P^{2}\right) \frac{\cos P r}{r} & =4 \pi \delta(\vec{x}), \\
-\vec{\partial}^{2} \frac{1}{r} & =4 \pi \delta(\vec{x}), \\
\left(-\vec{\partial}^{2}+B^{2}\right) \frac{e^{-|B| r}}{r} & =4 \pi \delta(\vec{x}) .
\end{aligned}
$$

The tangent $\mathbb{R}^{3}$-kernel (Laplacian kernel) as limit of spherical and hyperbolic potential is given by the Kepler potential:

$$
\frac{\cos P r}{r} \stackrel{P \rightarrow 0}{\longrightarrow} \frac{1}{r} \stackrel{B \leftarrow 0}{\longleftarrow} \frac{e^{-|B| r}}{r} .
$$

The three-dimensional potentials are distributions of one-dimensional position representation coefficients, i.e., of the compact and noncompact abelian Cartan subgroups:

$$
\begin{aligned}
& \mathbb{E}^{1} \cong \mathbb{R} \cong \mathcal{Y}^{1} \cong \mathbf{S O}_{0}(1,1), \Omega^{1} \cong \mathbf{S O}(2), \\
& \mathbb{E}^{1} \subset \mathbb{E}^{3}, \Omega^{1} \subset \Omega^{3}: \int \frac{d y d z}{2 \pi} \int \frac{d^{3} q}{2 \pi^{2}} \frac{1}{\vec{q}^{2}+i o-P^{2}} e^{i \vec{q} \vec{x}}=\int \frac{d q}{\pi} \frac{1}{q^{2}+i o-P^{2}} e^{i q x}=\frac{e^{-i|P x|}}{i|P|}, \\
& \mathcal{Y}^{1} \subset \mathcal{Y}^{3}: \quad \int \frac{d y d z}{2 \pi} \int \frac{d^{3} q}{2 \pi^{2}} \frac{1}{\vec{q}^{2}+B^{2}} e^{i \vec{q} \vec{x}}=\int \frac{d q}{\pi} \frac{1}{q^{2}+B^{2}} e^{i q x}=\frac{e^{-|B x|}}{|B|} .
\end{aligned}
$$

In contrast to one-dimensional abelian positions with the exponential representation coefficients:

$$
\text { for } \begin{aligned}
\mathbb{E}^{1} \text { and } \Omega^{1}: \int \frac{d q}{\pi} \frac{i|P|}{q^{2}+i o-P^{2}} e^{i q x}=e^{-i|P x|,} \quad\left(-\partial^{2}-1\right) e^{-i|x|} & =2 i \delta(x), \\
\left(-\partial^{2}-1\right) \cos x & =0, \\
& \left(-\partial^{2}-1\right) \sin |x|=-2 \delta(x), \\
\text { for } \mathcal{Y}^{1}: \int \frac{d q}{\pi} \frac{|B|}{q^{2}+B^{2}} e^{i q x}=e^{-|B x|,} \quad\left(-\partial^{2}+1\right) e^{-|x|} & =2 \delta(x),
\end{aligned}
$$

the pole order differentiates, for three-dimensional positions with nonabelian rotations, between subgroup representation distributions for interactions (potentials, simple momentum poles, exponential position densities), e.g. $\frac{e^{-|B| r}}{r}$, and singularity free representations functions for negative energy states (momentum dipoles), e.g. $e^{-|B| r}$ :

$$
\begin{aligned}
& \text { for } \Omega^{3}: \int \frac{d^{3} q}{\pi^{2}} \frac{i|P|}{\left(\vec{q}^{2}+i o-P^{2}\right)^{2}} e^{i \vec{q} \vec{x}}=e^{-i|P| r}=\frac{|P|}{2 i \pi} \frac{e^{-i|P| r}}{r} * \frac{e^{-i|P| r}}{r}, \\
& \text { for } \mathcal{Y}^{3}: \int \frac{d^{3} q}{\pi^{2}} \frac{|B|}{\left(\vec{q}^{2}+B^{2}\right)^{2}} e^{i \vec{q} \vec{x}}=e^{-|B| r}=\frac{|B|}{2 \pi} \frac{e^{-|B| r}}{r} * \frac{e^{-|B| r}}{r} .
\end{aligned}
$$

The residues are normalized with $\pi^{2}$. Simple hyperbolic poles at $\vec{q}^{2}=-1$ in three position dimensions give the Yukawa potential; dipoles, etc., give $r=0$-regular representation coefficients.

The derivations of spherical and hyperbolic representation coefficients lead to a spherical and hyperbolic (Yukawa) potential, respectively:

$$
\begin{aligned}
& \text { for } \mathbb{E}^{3}:\left(-\vec{\partial}^{2}-1\right) \frac{\sin r}{r}=0, \\
& \text { for } \Omega^{3}:\left(-\vec{\partial}^{2}-1\right) e^{-i r}=\frac{2 i}{r} e^{-i r}, \quad\left(-\vec{\partial}^{2}-1\right)^{2} e^{-i r}=8 \pi i \delta(\vec{x}), \\
& \text { for } \mathcal{Y}^{3}:\left(-\vec{\partial}^{2}+1\right) e^{-r}=\frac{2}{r} e^{-r}, \quad\left(-\vec{\partial}^{2}+1\right)^{2} e^{-r}=8 \pi \delta(\vec{x}) .
\end{aligned}
$$


The invariant "non-flat" derivations for the eigenvalues (invariants) $Q^{2}$ involve the nontrivial potential,

$$
\left(-\vec{\partial}^{2}+\frac{\partial}{\partial \frac{r^{2}}{4}}\right) e^{-i Q r}=\left(-\vec{\partial}^{2}-\frac{2 i Q}{r}\right) e^{-i Q r}=Q^{2} e^{-i Q r}, i Q=(i|P|,|B|) .
$$

The identity for the functions with hyperbolic invariant $\vec{q}^{2}=-B^{2}$ is the Schrödinger equation for the rotation invariant ground-state coefficient of the nonrelativistic hydrogen atom with the Coulomb potential in the Hamiltonian $\mathbf{H} \cong-\frac{1}{2} \vec{\partial}^{2}-\frac{1}{r}$ (more ahead):

$$
\begin{array}{ll}
\text { for } \mathbb{E}^{3}:-\vec{\partial}^{2} \frac{\sin |P| r}{r} & =P^{2} \frac{\sin |P| r}{r}, \\
\text { for } \Omega^{3}:\left(-\vec{\partial}^{2}-\frac{2 i|P|}{r}\right) e^{-i|P| r} & =P^{2} e^{-i|P| r}, \\
\text { for } \mathcal{Y}^{3}:\left(-\vec{\partial}^{2}-\frac{2|B|}{r}\right) e^{-|B| r} & =-B^{2} e^{-|B| r} .
\end{array}
$$

In general, for the hyperboloid $\mathcal{Y}^{s}$, the sphere $\Omega^{s}$ and the Euclidean space $\mathbb{E}^{s}$, the scalar distributions for the definite orthogonal local action group $\mathbf{O}(s)$ with hyperbolic singularities (invariants) $(i \vec{q})^{2}=1$ (positive definite Killing metric - with unit $\vec{q}^{2}=-B^{2}$ ) on imaginary spheres, and spherical ones $(i \vec{q})^{2}=-1$ (negative definite Killing metric - with unit $\vec{q}^{2}=P^{2}$ ) on real spheres $\Omega^{s-1}$ define the hyperbolic Macdonald functions $\mathcal{K}$ with, for imaginary argument, the corresponding spherical Hankel $\mathcal{H}^{1,2}=\mathcal{J} \pm i \mathcal{N}$ (Bessel $\mathcal{J}$ with Neumann $\mathcal{N}$ ) functions. Integrals with integer poles $\frac{s}{2}-v=1,2, \ldots$ are important for spacetime representations and will be defined in a form, amenable for an operational interpretation, by their Fourier transforms, i.e., in an analysis with $\mathbb{R}^{s} \ni \vec{x} \longmapsto e^{i \vec{q} \vec{x}} \in \mathbf{U}(1)$, collected at the invariant singularities $\vec{q}^{2}=0, \mp 1$ :

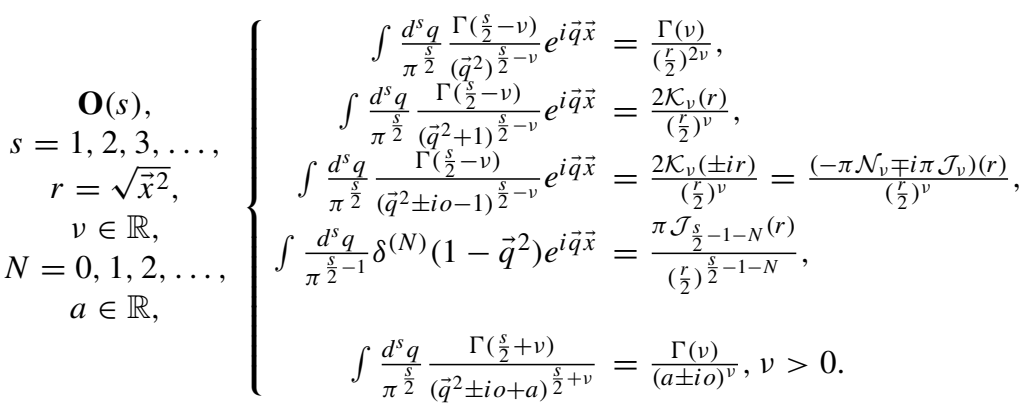

With a 2 -sphere spread for the index increase by one $v \longrightarrow v+1$ :

$$
\frac{\left(2 \mathcal{K}_{\nu+1}, \pi \mathcal{N}_{v+1}, \pi \mathcal{J}_{\nu+1}\right)(r)}{\left(\frac{r}{2}\right)^{v+1}}=-\frac{d}{d \frac{r^{2}}{4}} \frac{\left(2 \mathcal{K}_{\nu}, \pi \mathcal{N}_{\nu}, \pi \mathcal{J}_{\nu}\right)(r)}{\left(\frac{r}{2}\right)^{v}},
$$

all (half-)integer index functions above can be obtained as follows:

$$
\mathbb{R}_{+} \ni r \longmapsto \frac{\left(2 \mathcal{K}_{v}, \pi \mathcal{N}_{v}, \pi \mathcal{J}_{v}\right)(r)}{\left(\frac{r}{2}\right)^{v}}=\left\{\begin{array}{c}
\left(-\frac{d}{d \frac{r^{2}}{4}}\right)^{N} \sqrt{\pi}\left(e^{-r}, \sin r, \cos r\right), \\
v=N-\frac{1}{2}=-\frac{1}{2}, \frac{1}{2}, \frac{3}{2}, \ldots, \\
\left(-\frac{d}{d \frac{r^{2}}{4}}\right)^{N}\left(2 \mathcal{K}_{0}, \pi \mathcal{N}_{0}, \pi \mathcal{J}_{0}\right)(r), \\
v=N=0,1,2, \ldots,
\end{array}\right.
$$

The half-integer index functions are relevant for time or 3-position, in general for odd-dimensional manifolds $s=1+2 R=1,3, \ldots$, ( $R$ for rank) with definiteness $k=(1,-1)=\iota^{2}$ for compactness $\iota=(1, i)$ and hyperbolic and spherical invariant. They 
start with exponentials and (co)sines as coefficients of the real one-dimensional Lie groups $\mathbf{D}(1) \cong \mathbf{S O}_{0}(1,1) \cong \mathbb{R}$ and $\mathbf{U}(1) \cong \mathbf{S O}(2) \cong \mathbb{R} / \mathbb{Z}$.

The integer index functions are relevant for four-dimensional spacetime, in general for even-dimensional manifolds $s=2 R=2,4, \ldots$ ( $R$ for rank). The even-dimensional integrals integrate singularities of odd-dimensional ones, e.g.:

$$
\begin{aligned}
2 \mathcal{K}_{0}(r) & =\int \frac{d^{2} q}{\pi} \frac{1}{\vec{q}^{2}+1} e^{i \vec{q} \vec{x}}=\int d p \int \frac{d q}{\pi} \frac{1}{q^{2}+p^{2}+1} e^{i q r} \\
& =\int \frac{d p}{\sqrt{p^{2}+1}} e^{-\sqrt{p^{2}+1} r}=2 \int_{1}^{\infty} \frac{d \kappa}{\sqrt{\kappa^{2}-1}} e^{-\kappa r} .
\end{aligned}
$$

\section{Free Scattering for Euclidean Position}

Nonrelativistic scattering theory is formulated in terms of infinite-dimensional faithful unitary representations of the Euclidean group $\mathbf{S O}(3) \overrightarrow{\times} \mathbb{R}^{3}$ and of its rotation group classes, the Euclidean position $\mathbb{E}^{3}$. The solutions of the Schrödinger equation,

$$
\mathbf{H}=\frac{\overrightarrow{\mathbf{p}}^{2}}{2}, 0 \leq E=\frac{P^{2}}{2} \in \mathbf{s p e c}_{+} \mathbf{H}:\left(\vec{\partial}^{2}+P^{2}\right) \psi(\vec{x})=0,
$$

for trivial translation invariant $P=0$ are fixgroup $\mathbf{S O}(3)$-representations with harmonic polynomials, $\vec{\partial}^{2}(\vec{x})^{L}=0$. For nontrivial momentum $P>0$, the solutions are coefficients of the irreducible Hilbert space representations of the Euclidean group, induced by fixgroup SO(2)-representations, with dual SO(2)-eigenvalues $\pm h \in \mathbb{Z}$ (helicity) for axial rotations around the momentum direction $\frac{\vec{q}}{P}=\vec{\omega} \in \Omega^{2}$.

Induced representations of a group start from representations of an "appropriate" subgroup - for a semidirect affine group $G \overrightarrow{\times} \mathbb{R}^{n}, G \subseteq \mathbf{G L}(n, \mathbb{R})$, from irreducible representations of the normal subgroup $\mathbb{R}^{n}$ with the space(-time) translations, characterized by (energy-) momenta, and their fixgroups $H \subseteq G$ with cosets $G / H$ and a $G$-compatible projection-valued measure thereon.

Adding up, by integration, all unitary irreducible position translation representations:

$$
\mathbb{R}^{3} \ni \vec{x} \longmapsto e^{i \vec{q} \vec{x}} \in \mathbf{U}(1), \vec{q} \in \mathbb{R}^{3},
$$

with their momenta on a 2-sphere $\vec{q}^{2}=P^{2}, P>0$, one obtains spherical Bessel functions as representation coefficients of Euclidean position $\mathbb{E}^{3} \cong \mathbf{S O}(3) \overrightarrow{\times} \mathbb{R}^{3} / \mathbf{S O}(3)$ for free (asymptotic) nonrelativistic scattering states:

$$
\begin{aligned}
& \mathbb{E}^{3} \ni \vec{x} \longmapsto \int \frac{d^{3} q}{4 \pi P} 2 \delta\left(\vec{q}^{2}-P^{2}\right) e^{i \vec{q} \vec{x}}=-\frac{1}{\operatorname{Pr}} \frac{\partial}{\partial r} \int d q \delta\left(q^{2}-P^{2}\right) e^{i q r} \\
& =-\frac{1}{P^{2} r} \frac{\partial}{\partial r} \cos \operatorname{Pr}=\frac{\sin P r}{\operatorname{Pr}}=j_{0}(P r) \text { with } r=|\vec{x}|=\sqrt{\vec{x}^{2}},
\end{aligned}
$$

The normalization uses the finite surface $\left|\Omega^{2}\right|$ of the unit 2 -sphere $\Omega^{2} \cong \mathbf{S O}(3) / \mathbf{S O}(2)$ with fixgroup $\mathbf{S O}(2)$, parametrizing, via the momentum directions, the axial rotation group classes of the compact rotation group:

$$
\Omega^{2}=\left\{\vec{\omega}=\frac{\vec{q}}{P} \in \mathbb{R}^{3} \mid \vec{q}^{2}=P^{2}>0\right\},\left|\Omega^{2}\right|=\int d^{3} q 2 \delta\left(\vec{q}^{2}-1\right)=4 \pi .
$$


The scalar Euclidean position functions above integrate the irreducible translation representation coefficients for the momentum directions $\vec{\omega} \in \Omega^{2}$ on the unit 2-sphere $(\mathcal{M}$ for Radon measure) where the 2 -sphere characterizes the orbits of a nontrivial momentum with "length" $P$ :

$$
\operatorname{SO}(3) \overrightarrow{\times} \mathbb{R}^{3}:\left\{\begin{aligned}
\mathbb{E}^{3} \ni \vec{x} \longmapsto & \int \frac{d^{3} q}{2 \pi P} \delta\left(\vec{q}^{2}-P^{2}\right) e^{i \vec{q} \vec{x}} \\
& =\int_{\Omega^{2}} \frac{d^{2} \omega}{\left|\Omega^{2}\right|} e^{i P \vec{\omega} \vec{x}}=\int_{\Omega^{2}} \frac{d^{2} \omega}{\left|\Omega^{2}\right|} \cos P \vec{\omega} \vec{x} \\
\text { for } \vec{q} & =|\vec{q}| \vec{\omega} \text { with } \vec{\omega}=\left(\begin{array}{c}
\cos \theta \\
\sin \theta \cos \phi \\
\sin \theta \sin \phi
\end{array}\right) \in \Omega^{2} \subset \mathbb{R}^{3}, \\
d^{2} \omega & =\sin \theta d \theta d \phi \in \mathcal{M}\left(\Omega^{2}\right)_{+},\left|\Omega^{2}\right|=4 \pi .
\end{aligned}\right.
$$

In the following for short $\int_{\Omega^{2}} d^{2} \omega=\int d^{2} \omega$.

The rotation representations for $\Omega^{2} \cong \mathbf{S O}(3) / \mathbf{S O}(2)$ are harmonically analyzable by the spherical harmonics $\left\{\vec{\omega}=\frac{\vec{x}}{r} \longmapsto \mathrm{Y}_{m}^{L}(\vec{\omega})=\left(\frac{\vec{x}}{r}\right)_{m}^{L}|L=0,1, \ldots ;| m \mid \leq L\right\}$ as orthonormal Hilbert basis of the 2-sphere functions $L^{2}\left(\Omega^{2}\right)$, decomposable into Hilbert spaces with irreducible action of the rotations:

$$
L^{2}\left(\Omega^{2}\right)=\bigoplus_{L=0}^{\infty} \mathbb{C}^{1+2 L}\left(\Omega^{2}\right)
$$

The radial coefficients of plane waves define the spherical Bessel functions $j_{L} \in$ $L^{\infty}\left(\mathbb{R}_{+}\right)$in the expansion of the irreducible translation $\mathbb{R}^{3}$-representation coefficient with repect to representations of the Euclidean group $\mathbf{S O}(3) \overrightarrow{\times} \mathbb{R}^{3}$. The essentially bounded representation coefficients of the Euclidean group in $L^{\infty}\left(\mathbb{R}^{3}\right)$ are products $Y_{m}^{L}(\phi, \theta) j_{L}(P r)$ of matching spherical Bessel functions and spherical harmonics:

$$
\begin{aligned}
P>0: \quad \mathbb{E}^{3} \ni \vec{x} \longmapsto & \int \frac{d^{3} q}{2 \pi P}\left(\begin{array}{l}
1 \\
i \frac{\vec{q}}{|\vec{q}|}
\end{array}\right) \delta\left(\vec{q}^{2}-P^{2}\right) e^{i \vec{q} \vec{x}} \\
& =\left(\begin{array}{l}
j_{0}(P r) \\
\frac{\vec{x}}{r} j_{1}(P r)
\end{array}\right)=\left(\begin{array}{l}
\frac{\sin P r}{P r} \\
\frac{\vec{x}}{r} \frac{\sin P r-P r \cos P r}{P^{2} r^{2}}
\end{array}\right), \\
i^{L} Y_{m}^{L}(\phi, \theta) j_{L}(r) & \sim\left(i \frac{\vec{x}}{2}\right)^{L} \frac{\sqrt{\pi}}{2} \frac{\mathcal{J}_{L+\frac{1}{2}}(r)}{\left(\frac{r}{2}\right)^{L+\frac{1}{2}}}=\left(i \frac{\vec{x}}{2}\right)^{L}\left(-\frac{\partial}{\partial \frac{r^{2}}{4}}\right)^{L} \frac{\sin r}{r} \\
& =(-i \vec{\partial})^{L} \frac{\sin r}{r}=\int \frac{d^{3} q}{2 \pi}(\vec{q})^{L} \delta\left(\vec{q}^{2}-1\right) e^{i \vec{q} \vec{x}} .
\end{aligned}
$$


A scalar quantum field of Euclidean position $\mathbb{E}^{3}=\mathbf{S O}(3) \overrightarrow{\times} \mathbb{R}^{3} / \mathbf{S O}(3)$ parametrizes the scalar Euclidean group $\mathbf{S O}(3) \overrightarrow{\times} \mathbb{R}^{3}$-coefficients for momentum invariant $P$ by the groundstate value of the anticommutator for a commutator-quantized field:

$$
\begin{aligned}
\mathbb{E}^{3} \ni \vec{x} \longmapsto \mathbf{E}(\vec{x}) & =\oplus \int \frac{d^{2} \omega}{\left|\Omega^{2}\right|}\left[e^{i \vec{q} \vec{x}} \mathrm{u}(\vec{\omega})+e^{-i \vec{q} \vec{x}} \mathrm{u}^{*}(\vec{\omega})\right], \\
& \text { with }|\vec{q}|=P, \\
\text { quantization: }\left[\mathrm{u}^{*}\left(\vec{\omega}^{\prime}\right), \mathrm{u}(\vec{\omega})\right] & =\mathbf{1}\left|\Omega^{2}\right| \delta\left(\vec{\omega}-\vec{\omega}^{\prime}\right), \\
\left\langle 0\left\{\mathrm{u}^{*}\left(\vec{\omega}^{\prime}\right), \mathrm{u}(\vec{\omega})\right\}\right\rangle 0 & =\left\langle 0\left|\mathrm{u}^{*}\left(\vec{\omega}^{\prime}\right) \mathrm{u}(\vec{\omega})\right| 0\right\rangle \\
& =\left\langle P^{2}, 0 ; \vec{\omega}^{\prime} \mid P^{2}, 0 ; \vec{\omega}\right\rangle=\left|\Omega^{2}\right| \delta\left(\vec{\omega}-\vec{\omega}^{\prime}\right), \\
\text { distributive Hilbert basis: } & \left\{\mathrm{u}(\vec{\omega})|0\rangle=\left|P^{2}, 0 ; \vec{\omega}\right\rangle \mid \vec{\omega} \in \Omega^{2}\right\}, \\
\text { translation action: } \vec{x} \bullet\left|P^{2}, 0 ; \vec{\omega}\right\rangle= & e^{i P \vec{\omega} \vec{x}}\left|P^{2}, 0 ; \vec{\omega}\right\rangle, \\
\mathbb{E}^{3} \ni \vec{x}-\vec{y} \longmapsto\langle 0|\mathbf{E}(\vec{y}) \mathbf{E}(\vec{x})| 0\rangle & =\int \frac{d^{3} q}{2 \pi P} \delta\left(\vec{q}^{2}-P^{2}\right) e^{i \vec{q}(\vec{x}-\vec{y})} .
\end{aligned}
$$

Its harmonic expansion integrates, with the normalized invariant measure $\frac{d^{2} \omega}{4 \pi}$ of the 2sphere $\Omega^{2} \cong \mathbf{S O}(3) / \mathbf{S O}(2)$, translation and axial rotation eigenoperators for momentum direction $\vec{\omega}$ as distributive basis of the representation Hilbert space.

The generalization with respect to position dimensions $s=2,3, \ldots$ is obvious: The faithful representations of the Euclidean group $\mathbf{S O}(s) \overrightarrow{\times} \mathbb{R}^{s}$ are characterized by momentum

invariant $P^{2}$ and irreducible fixgroup $\mathbf{S O}(s-1)$ representations. They start with Bessel functions as scalar coefficients:

$$
P>0: \mathbb{E}^{s} \ni \vec{x} \longmapsto \int \frac{d^{s} q}{\pi^{\frac{s}{2}-1} P^{s-2}} \delta\left(\vec{q}^{2}-P^{2}\right) e^{i \vec{q} \vec{x}}=\frac{\pi \mathcal{J}_{\frac{s}{2}-1}(P r)}{\left(\frac{P r}{2}\right)^{\frac{s}{2}-1}}
$$

With the polar decomposition $\mathbb{R}^{s}=\mathbb{R}_{+} \times \Omega^{s-1}$, the Hilbert space for fixed momentum $P>0$ is given by the square integrable function $L^{2}\left(\Omega^{s-1}\right)$ on the $(s-1)$-sphere. The harmonic analysis with respect to the Euclidean group works with the product Hilbert space $L^{2}\left(\mathbb{R}_{+}\right) \times L^{2}\left(\Omega^{s-1}\right) \cong L^{2}\left(\mathbb{R}^{s}\right)$.

\section{Atomic Spectrum for Kepler Position}

The Kepler dynamics with time translation generator,

$$
\mathbf{H}=\frac{\overrightarrow{\mathbf{p}}^{2}}{2}+\frac{\epsilon}{\mathbf{r}}, \epsilon= \pm 1 \text { repulsive, attractive, }
$$

has, for an attraction, both negative energy (imaginary momentum, bound) waves and positive energy (real momentum, scattering) solutions. The noncompact position as represented by the wave functions of the nonrelativistic hydrogen atom is not flat Euclidean position $\mathbb{E}^{3}$, but maximally symmetric hyperbolic position $\mathcal{Y}^{3} \cong \mathbf{S O}_{0}(1,3) / \mathbf{S O}(3)$, parametrizing the rotation group classes in the Lorentz group. $\mathbb{E}^{3}$ and $\mathcal{Y}^{3}=\exp \mathbb{R}^{3}$ are $\mathbb{R}^{3}$-isomorphic as manifolds, not as homogeneous spaces. 
The nonrelativistic Kepler dynamics has, in addition to the rotation symmetry, a LenzRunge "perihelion" invariance which is the reason for the "periodicity" of the "periodic" system, i.e., of the atomic spectrum. Angular momentum $\overrightarrow{\mathbf{J}}=i \overrightarrow{\mathbf{x}} \times \overrightarrow{\mathbf{p}}$ and Lenz-Runge vector $\overrightarrow{\mathbf{R}}=i\left[\overrightarrow{\mathbf{p}}, \frac{\overrightarrow{\boldsymbol{J}}^{2}}{2}+\epsilon \mathbf{r}\right]$ are conserved $[\overrightarrow{\mathbf{J}}, \mathbf{H}]=0=[\overrightarrow{\mathbf{R}}, \mathbf{H}]$ and orthogonal to each other $\overrightarrow{\mathbf{J}} \overrightarrow{\mathbf{R}}=0$ (trivial invariant). With the sum and difference $\overrightarrow{\mathbf{J}}_{ \pm}=\frac{1}{2}\left(\overrightarrow{\mathbf{J}} \pm \frac{\overrightarrow{\mathbf{R}}}{\sqrt{-2 \mathbf{H}}}\right)$ for negative energies the Hamiltonian can be written as $2\left(\overrightarrow{\mathbf{J}}_{+}^{2}+\overrightarrow{\mathbf{J}}_{-}^{2}\right)=1+\frac{1}{2 \mathbf{H}}$. The orthogonality of angular momentum and Lenz-Runge vectors involves the equality of the invariants $\overrightarrow{\mathbf{J}}_{+}^{2}=$ $\overrightarrow{\mathbf{J}}_{-}^{2}=-J(J+1)$ in the energy $-4 J(J+1)=1+\frac{1}{2 E}$.

The equations for the Kepler potential have $(r=0)$-regular solutions with confluent hypergeometric functions $\mathbb{C} \ni z \longmapsto{ }_{1} \mathrm{~F}_{1}(\alpha ; \gamma ; z), \alpha, \gamma \in \mathbb{C}$ :

$$
\begin{aligned}
& {\left[\vec{\partial}^{2}-2 V(r)+2 E\right] \psi(\vec{x})=0,} \\
& \psi(\vec{x})=\sum_{L=0}^{\infty} \sum_{m=-L}^{L}(\vec{x}){ }_{m}^{L} d_{L}(r) \Rightarrow\left[d_{r}^{2}+\frac{2(1+L)}{r} d_{r}-2 V(r)+2 E\right] d_{L}(r)=0, \\
& V(r)=\frac{\epsilon}{r}: d_{L}(r)={ }_{1} \mathrm{~F}_{1}\left(1+L+\frac{\epsilon}{i Q} ; 2+2 L ; 2 i Q r\right) e^{-i Q r} \text { for } i Q=|B| \leftrightarrow i P, \\
& {\left[\zeta d_{\zeta}^{2}+(\gamma-\zeta) d_{\zeta}-\alpha\right]_{1} \mathrm{~F}_{1}(\alpha ; \gamma ; \zeta)=0,{ }_{1} \mathrm{~F}_{1}(\alpha ; \gamma ; \zeta)=\sum_{k=0}^{\infty} \frac{\Gamma(\alpha+k)}{\Gamma(\alpha)} \frac{\Gamma(\gamma)}{\Gamma(\gamma+k)} \frac{\zeta^{k}}{k !} .}
\end{aligned}
$$

Negative energy (bound) state and positive energy (scattering) solutions are related to each other by the noncompact-compact relation $|B| \leftrightarrow i|P|$ of the hyperbolic $\mathbf{S O}_{0}(1,1)$ invariant. For the attractive interaction $V(r)=-\frac{1}{r}$ and states with negative energy (binding energy) and square-integrable hyperbolic wave functions, the radial imaginary

"momentum" and, therefore, the energy is "quantized" — the related noncompact invariant is determined by the compact ones with the degree of the Laguerre polynomials L:

$$
\begin{aligned}
& d_{L}(r) \sim{ }_{1} \mathrm{~F}_{1}\left(1+L-\frac{1}{|B|} ; 2+2 L ; 2|B| r\right) e^{-|B| r} \\
& \sim \mathrm{L}_{N}^{1+2 L}(2|B| r) e^{-|B| r}, \\
& Q^{2}=-1, B^{2}<0, \Rightarrow\left\{\begin{array}{rl}
1+2 \\
E
\end{array}=-\frac{B^{2}}{2},\right. \\
& \mathbb{N} \ni \frac{1}{B}=n=1+2 J=1+L+N, L, N=0,1, \ldots, \\
& \psi_{L m}^{2 J}(\vec{x}) \sim\left(\frac{\vec{x}}{n}\right)_{m}^{L} \mathrm{~L}_{N}^{1+2 L}\left(\frac{2 r}{n}\right) e^{-\frac{r}{n}}, \\
& \psi_{E}(t)=e^{i E t}|E>,| E>=\mid J>, J ; L, m \cong \psi_{L m}^{2 J} \in L^{2}\left(\mathbb{R}^{3}\right) .
\end{aligned}
$$

The product of spatially decreasing exponentials, characterized by the noncompact "momentum" invariants $B$, the harmonic $\mathbf{S O}(3)$-polynomials of degree $L$ and the Laguerre polynomials with degree $N=2 J-L$ (knot number) give square integrable wave functions. For fixed principal quantum number $n=1+2 J$, i.e., for energy $E=-\frac{1}{2 n^{2}}$, the products of Laguerre and harmonic SO(3)-polynomials give $n^{2}$ harmonic $\mathbf{S O}(4)$-polynomials (more ahead).

In contrast to the negative energy (bound)-state exponential $e^{-|B| r}$, the hyperbolic scattering solutions in the Kepler potential $V(r)=\frac{\epsilon}{r}$ - not leveling off stronger than $\frac{1}{r}-$ with positive energy and real momentum from a continuous spectrum, are obtained after the 
separation of a unitary exponential $e^{i P r}$. The radial equations have the real $(r=0)$-regular solutions with confluent hypergeometric functions:

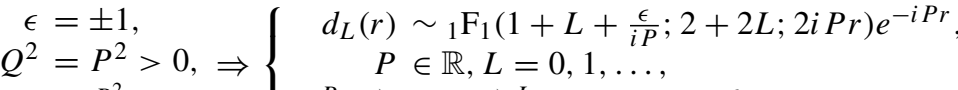

$$
\begin{aligned}
& E=\frac{P^{2}}{2}, \quad \quad \quad \psi_{L m}^{P}(\vec{x}) \sim(P \vec{x})_{m 1}^{L} \mathrm{~F}_{1}\left(1+L+\frac{\epsilon}{i P} ; 2+2 L ; 2 i P r\right) e^{-i P r} .
\end{aligned}
$$

The large distance behavior of the hyperbolic scattering solutions does not lead to spherical Bessel functions for free particles, representing flat Euclidean position $\mathbb{E}^{3}$. For the curved hyperbolic position $\mathcal{Y}^{3}$-representations, the asymptotic Bessel functions $j_{L}(r) \rightarrow$ $\frac{\sin \left(r-\frac{L \pi}{2}\right)}{r}$ are modified by a radial-dependent logarithm and a constant phase $\alpha_{L}$ :

$$
\begin{aligned}
r \rightarrow \infty:(P \vec{x})_{m}^{L}{ }_{1} \mathrm{~F}_{1}\left(1+L+\frac{\epsilon}{i P}\right. & ; 2+2 L ; 2 i P r) e^{-i P r} \\
& \rightarrow \mathrm{Y}_{m}^{L}(\phi, \theta) \frac{\sin \left(P r-\frac{L \pi}{2}-\frac{\epsilon}{P} \log 2 P r+\alpha_{L}\right)}{\operatorname{Pr}} .
\end{aligned}
$$

In contrast to the representations of flat Euclidean space (scattering waves, free particles, simple momentum poles), Hilbert representations of three-dimensional hyperbolic position $\mathcal{Y}^{3}=e^{\mathbb{R}^{3}} \ni e^{\vec{\sigma} \vec{x}} \leftrightarrow \vec{x} \in \mathbb{R}^{3}$ (isomorphic as manifolds) with nonabelian degrees of freedom use momentum dipoles. The Hilbert space $L^{2}\left(\Omega^{2}\right)$ on the momentum orientation sphere $\frac{\vec{q}}{|\vec{q}|} \in \Omega^{2}$ for nonrelativistic scattering differs from the Hilbert space $L^{2}\left(\mathcal{Y}^{3}\right)$ on hyperbolic position $\vec{x} \in \mathcal{Y}^{3}$.

The measure of the unit 3-sphere as the orientation manifold of the rotation group SO(3) in the invariance group SO(4) for negative energy (bound) waves has a momentum parametrization by an imaginary dipole $\vec{q}^{2}=-1$ :

$$
\frac{1}{\sqrt{\vec{q}^{2}+1}}\left(\begin{array}{c}
1 \\
\vec{q}
\end{array}\right) \in \Omega^{3} \subset \mathbb{R}^{4} \Rightarrow\left|\Omega^{3}\right|=\int d^{3} \omega=\int d^{3} q \frac{2}{\left(\vec{q}^{2}+1\right)^{2}}=2 \pi^{2} .
$$

A curvature unit (inverse length) $|B|$ is implemented by $\int d^{3} q \frac{2}{\left(\vec{q}^{2}+B^{2}\right)^{2}}=\frac{2 \pi^{2}}{|B|}$.

The Fourier-transformed $\Omega^{3}$-measure gives the hydrogen ground-state coefficient with hyperbolic invariant as a scalar representation coefficient of 3-position space:

$$
\mathbf{S O}_{0}(1,3) / \mathbf{S O}(3) \cong \mathcal{Y}^{3} \cong \mathbb{R}^{3} \ni \vec{x} \longmapsto \int \frac{d^{3} q}{\pi^{2}} \frac{|B|}{\left(\vec{q}^{2}+B^{2}\right)^{2}} e^{i \vec{q} \vec{x}}=e^{-|B| r} .
$$

It involves the half-integer index Macdonald function for $N=0$ :

$$
\begin{aligned}
\int \frac{d^{3} q}{\pi^{2}} \frac{\Gamma(2+N)}{\left(\vec{q}^{2}+1\right)^{2+N}} e^{i \vec{q} \vec{x}} & =\frac{1}{\sqrt{\pi}}\left(\frac{r}{2}\right)^{\frac{1}{2}+N} 2 \mathcal{K}_{\frac{1}{2}+N}(r)=\left(\frac{r}{2}\right)^{1+2 N}\left(-\frac{d}{d} \frac{r^{2}}{4}\right)^{1+N} e^{-r}, \\
\text { for } N & =-1,0,1,2, \ldots
\end{aligned}
$$

In the negative energy (bound) waves, position as a noncompact hyperboloid is represented in the form of Fourier-transformed $\Omega^{3}$-measures with a continuous invariant $B^{2}$ for the imaginary "momenta" $\vec{q}^{2}=-B^{2}$ on a 2 -sphere $\Omega^{2}$.

The rotation dependence $\vec{x}$ is effected by momentum derivation $i \frac{\partial}{\partial \vec{q}}$ of the $\Omega^{3}$-measure:

$$
i \vec{x} e^{-r}=\int \frac{d^{3} q}{\pi^{2}} \frac{4 \vec{q}}{\left(\vec{q}^{2}+1\right)^{3}} e^{i \vec{q} \vec{x}} \text { with } \frac{4 \vec{q}}{\left(\vec{q}^{2}+1\right)^{3}}=-\frac{\partial}{\partial \vec{q}} \frac{1}{\left(\vec{q}^{2}+1\right)^{2}} .
$$


The 3-vector factor $\frac{2 \vec{q}}{\vec{q}^{2}+1}=\frac{\vec{q}}{|\vec{q}|} \sin \chi$ is uniquely supplemented to a parametrization of the unit 3-sphere by a normalized 4-vector:

$$
\left(\begin{array}{c}
\cos \chi \\
\frac{\vec{q}}{|\vec{q}|} \sin \chi
\end{array}\right)=\frac{1}{\vec{q}^{2}+1}\left(\begin{array}{c}
1-\vec{q}^{2} \\
2 \vec{q}
\end{array}\right)=\left(\begin{array}{c}
p_{0} \\
\vec{p}
\end{array}\right)=p \in \Omega^{3} \subset \mathbb{R}^{4}, p_{0}^{2}+\vec{p}^{2}=1 .
$$

The unit 4-vector $\mathrm{Y}^{\left(\frac{1}{2}, \frac{1}{2}\right)}(p) \sim\left(\begin{array}{c}p_{0} \\ \vec{p}\end{array}\right) \in \Omega^{3}$ is the analogue to the unit 3-vector $\mathrm{Y}^{1}\left(\frac{\vec{q}}{|\vec{q}|}\right) \sim$ $\frac{\vec{q}}{|\vec{q}|} \in \Omega^{2}$ used for the buildup of the 2-sphere harmonics $\mathrm{Y}^{L}\left(\frac{\vec{q}}{|\vec{q}|}\right) \sim\left(\frac{\vec{q}}{|\vec{q}|}\right)^{L}$. Analoguously, the $\Omega^{3}$-harmonics are the totally symmetric traceless products $\mathrm{Y}^{(J, J)}(p) \sim(p)^{2 J}$, e.g., for $J=1$, the nine independent components in the $(4 \times 4)$-matrix and the decomposition $9 \stackrel{\text { SO(3) }}{=} 1 \oplus 3 \oplus 5$ with the respective components $p_{0}^{2}-\frac{1}{4}, p_{0} \vec{p}$, and $p_{a} p_{b}-\frac{\delta_{a b}}{3} \vec{p}^{2}$ :

$$
\begin{aligned}
\mathrm{Y}^{(1,1)}(p) \sim(p)_{j k}^{2} & =p_{j} p_{k}-\frac{\delta_{j k}}{4} \cong\left(\begin{array}{c|c}
p_{0}^{2}-\frac{1}{4} & p_{0} p_{a} \\
\hline p_{0} p_{b} & p_{a} p_{b}-\frac{\delta_{a b}}{4}
\end{array}\right), \\
\text { with } p_{a} p_{b}-\frac{\delta_{a b}}{4} & =p_{a} p_{b}-\frac{\delta_{a b}}{3} \vec{p}^{2}-\frac{\delta_{a b}}{3}\left(p_{0}^{2}-\frac{1}{4}\right) \text { for } p^{2}=1 .
\end{aligned}
$$

The Kepler negative energy (bound) waves in $(1+2 J)^{2}$-multiplets for $\mathbf{S O}(4)$ come with momentum poles of order $2+2 J$ :

$$
\begin{aligned}
\frac{1}{B}= & 1+2 J: \mathcal{Y}^{3} \ni \vec{x} \longmapsto \int \frac{d^{3} q}{\pi^{2}} \frac{1}{\left(\vec{q}^{2}+1\right)^{2}}(p)^{2 J} e^{i \vec{q} B \vec{x}} \\
= & \int \frac{d^{3} q}{\pi^{2}} \frac{1}{\left(\vec{q}^{2}+1\right)^{2+2 J}}\left(\begin{array}{l}
1-\vec{q}^{2} \\
2 \vec{q}
\end{array}\right)^{2 J} e^{i \vec{q} B \vec{x}} \\
& \text { with } p=\frac{1}{\vec{q}^{2}+1}\left(\begin{array}{l}
1-\vec{q}^{2} \\
2 \vec{q}
\end{array}\right), \text { and } E=-\frac{B^{2}}{2} .
\end{aligned}
$$

The hyperboloid curvature units (binding energies) $-2 E_{2 J}=\frac{1}{(1+2 J)^{2}}$ involve the Plancherel density $d_{(J, J)}=(1+2 J)^{2}$ for $\mathbf{S O}(4)$, modified, in the hydrogen atom $\frac{1}{(1+2 J)^{2} l_{\text {Bohr }}^{2}}$ by the Bohr length $l_{B o h r}=\frac{\hbar}{\alpha_{\text {Som }} c m_{e}} \sim 0.5 \times 10^{-10} \mathrm{~m}$ as intrinsic unit. The nonrelativistic hydrogen spectrum has both a noncompact and a compact structure: The energy $E_{0}=-\frac{B_{0}^{2}}{2}$ of the ground-state $(0,0)$ is taken from an $\mathbf{S O}_{0}(1,1)$-related continuous spectrum. The SO(4)-nontrivial states $(J, J)$ as symmetric product states of $\left(\frac{1}{2}, \frac{1}{2}\right)$ are characterized by the discrete numbers $(1+2 J)$ with the noncompact invariants $B^{2}=\frac{B_{0}^{2}}{(1+2 J)^{2}}$ for the product representations:

$$
\mathcal{Y}^{3} \ni \vec{x} \longmapsto \int \frac{d^{3} q}{\pi^{2}} \frac{B_{0}}{\left(\vec{q}^{2}+B_{0}^{2}\right)^{2}}(p)^{2 J} e^{\frac{i \vec{q} \vec{x}}{1+2 J}} \text {, with } p=\frac{1}{\vec{q}^{2}+B_{0}^{2}}\left(\begin{array}{l}
B_{0}^{2}-\vec{q}^{2} \\
2 B_{0} \vec{q}
\end{array}\right) .
$$

The Fourier transformations with the 3 -sphere measure,

$$
\mu(\vec{x}) e^{-r}=\int \frac{d^{3} q}{\pi^{2}} \frac{1}{\left(\vec{q}^{2}+1\right)^{2}} \tilde{\mu}(\vec{q}) e^{i \vec{q} \vec{x}},
$$

are used for the ground-state singlet with a dipole,

$$
\begin{aligned}
J=0, \frac{1}{B}=1: e^{-B r}=\int \frac{d^{3} q}{\pi^{2}} \frac{B}{\left(B^{2}+\vec{q}^{2}\right)^{2}} e^{i \vec{q} \vec{x}} & =\int \frac{d^{3} q}{\pi^{2}} \frac{1}{\left(\vec{q}^{2}+1\right)^{2}} e^{i \vec{q} B \vec{x}}, \\
\left(-\vec{\partial}^{2}+B^{2}\right)^{2} e^{-B r} & =8 \pi B \delta(\vec{x}) .
\end{aligned}
$$


for the negative energy (bound)-state quartet with $\mathrm{Y}^{\left(\frac{1}{2}, \frac{1}{2}\right)}(p) \sim(p)^{1}$ and tripole, leading to Laguerre polynomials:

$$
\begin{aligned}
J=\frac{1}{2}, \frac{1}{B}=2: \int \frac{d^{3} q}{\pi^{2}} \frac{1}{\left(\vec{q}^{2}+1\right)^{2}}\left(\begin{array}{l}
p_{0} \\
\vec{p}
\end{array}\right) e^{i \vec{q} B \vec{x}} & =\int \frac{d^{3} q}{\pi^{2}} \frac{1}{\left(\vec{q}^{2}+1\right)^{3}}\left(\begin{array}{l}
1-\vec{q}^{2} \\
2 \vec{q}
\end{array}\right) e^{i \vec{q} B \vec{x}} \\
& =\left(\begin{array}{c}
\frac{B r-1}{2} \\
i \frac{B \vec{x}}{2}
\end{array}\right) e^{-B r}=\left(\begin{array}{c}
-\frac{1}{4} \mathrm{~L}_{1}^{1}(2 B r) \\
i B \vec{x} \frac{1}{2} \mathrm{~L}_{0}^{3}(2 B r)
\end{array}\right) e^{-B r},
\end{aligned}
$$

and for the negative energy (bound)-state nonet with $\mathrm{Y}^{(1,1)}(p) \sim(p)^{2}$ and quadrupole:

$$
\begin{aligned}
J=1, \frac{1}{B} & =3: \int \frac{d^{3} q}{\pi^{2}} \frac{1}{\left(\vec{q}^{2}+1\right)^{2}}\left(\begin{array}{l}
p_{0}^{2}-\frac{1}{4} \\
p_{0} \vec{p} \\
\vec{p} \otimes \vec{p}-\frac{\mathbf{1}_{3}}{3} \vec{p}^{2}
\end{array}\right) e^{i \vec{q} B \vec{x}} \\
& =\int \frac{d^{3} q}{\pi^{2}} \frac{4}{\left(\vec{q}^{2}+1\right)^{4}}\left(\begin{array}{l}
3\left(\frac{1-\vec{q}^{2}}{4}\right)^{2}-\frac{\vec{q}^{2}}{4} \\
\vec{q} \frac{1-\vec{q}^{2}}{2} \\
\vec{q} \otimes \vec{q}-\frac{\mathbf{1}_{3}}{3} \vec{q}^{2}
\end{array}\right) e^{i \vec{q} B \vec{x}}=\left(\begin{array}{l}
\frac{1}{4}\left(1-2 B r+\frac{2 B^{2} r^{2}}{3}\right) \\
i B \vec{x} \frac{B r-2}{6} \\
-\frac{B^{2}}{6}\left(\vec{x} \otimes \vec{x}-\mathbf{1}_{3} \frac{r^{2}}{3}\right)
\end{array}\right) e^{-B r} \\
& =\left(\begin{array}{l}
\frac{1}{12} \mathrm{~L}_{2}^{1}(2 B r) \\
-i B \vec{x} \frac{1}{12} \mathrm{~L}_{1}^{3}(2 B r) \\
-B^{2}\left(\vec{x} \otimes \vec{x}-\mathbf{1}_{3} \frac{r^{2}}{3}\right) \frac{1}{6} \mathrm{~L}_{0}^{5}(2 B r)
\end{array}\right) e^{-B r} .
\end{aligned}
$$

The Kepler representation $(J, J)$ of $\mathbf{S O}(4)$ as the highest-dimensional representation in the totally symmetric product of the defining four-dimensional representation $\bigvee^{2 J}\left(\frac{1}{2}, \frac{1}{2}\right) \cong$ $(J, J) \oplus \ldots$ is decomposable with respect to the position rotation $\mathbf{S O}(3)$-properties into irreducible representations of dimension $(1+2 L)$ with integer $L=0,1, \ldots$ for angular momentum $\overrightarrow{\mathbf{J}}=\overrightarrow{\mathbf{J}}_{+}+\overrightarrow{\mathbf{J}}_{-}$:

$$
\begin{aligned}
(J, J) & \stackrel{\mathbf{S O}(3)}{\cong} \bigoplus_{L=0,1, \ldots, 2 J}(L),(1+2 J)^{2}=\sum_{L=0}^{2 J}(1+2 L), \\
2 J=L+N & \Rightarrow(L, N)=(2 J, 0),(2 J-1,1), \ldots,(0,2 J) .
\end{aligned}
$$

With semisimple rank two Lie algebra, the irreducible SO(4)-representations are characterized by two invariants. The difference $2 J-L=N$ characterizing the classes $\mathbf{S O}(4) / \mathbf{S O}(3) \cong \Omega^{3}$ is the radial quantum number or knot number. It is the degree of the Laguerre polynomials in the factorization of the Fourier-transformed harmonic SO(4)polynomials by harmonic SO(3)-polynomials and SO(3)-invariant Laguerre polynomials:

$$
\begin{aligned}
\int \frac{d^{3} q}{\pi^{2}} \frac{B}{\left(\vec{q}^{2}+B^{2}\right)^{2}}\left(\frac{p}{B}\right)^{2 J} e^{i \vec{q} \vec{x}} \\
\quad \Rightarrow\left\{(B \vec{x})^{L} \mathrm{~L}_{N}^{1+2 L}(2 B r) e^{-B r} \mid L, N=0,1, \ldots, L+N=2 J\right\} .
\end{aligned}
$$

As seen in experiments, there is, in addition to the Lenz-Runge SO(4), a twofold degeneracy in the atoms. It originates from an "internal" spin $\mathbf{S U}(2)$-property of the electron not contained in the nonrelativistic scheme above. It can be added by an ad hoc doubling leading to doubled multiplicities $2 n^{2}=2,8,18, \ldots$.

Obviously, the SO(4)-periodicity for the Kepler-potential negative energy (bound) states is strictly valid only for the nonrelativistic hydrogen atom. It is modified by relativistic corrections and can serve as a first order approximation for the other atoms. In the periodic 
system of the atoms, additional interactions are relevant which lead to more than one not completely filled shell, etc.

\section{Operational Spacetimes}

The particle concept (with Wigner's definition) — sufficient for the asymptotics without interaction (flat spacetime approach) - is a spacetime projection on familiar pictures, therefore both useful and restricted. Today's quantum theory is a special relativistic (Minkowskian) quantum field theory. The concept of a field as used in the standard model of electroweak and strong interactions is more basic than that of a particle: All particles are embedded in fields, but not all fields are parametrizable completely by particle structures. This holds especially for mass zero fields as used for the electroweak and gravitational interactions, and for strong interaction parametrizing quarks and gluons, if confined. Apparently, not all interactions are harmonically analyzable by unitary representations of the Poincaré group and its Lorentz group classes, i.e., of Minkowski spacetime.

For relativistic scattering, the perturbative expansion of an interaction by particle embedding Feynman propagators with simple energy-momentum poles encounters "divergencies" which can be sedated, in "renormalizable" theories, by the regularization-renormalization procedure. The price to be paid is the principal indeterminacy of some basic physical properties like mass ratios and coupling constants. To parametrize a propagator, as used in Feynman diagrams for a perturbation expansion of field theory, only by "real" particles is incomplete, as seen, e.g., in the non-particle implemented Coulomb interaction, coming together with the two polarized photonic degrees of freedom in the four-component electromagnetic field. To completize "real" or "on-shell" particles, and to include interactions, the slippery concept of "virtual" or "off-shell" particles is introduced. The causally supported simple pole off-shell contributions cause the "divergencies."

With the requirement of local inertia systems, spacetimes as used in general relativity are postulated to be Riemannian manifolds with a causal signature (1,3)-metric, determined by the energy-momentum tensor. With Dirac and Heisenberg: Particles cannot explain particles - in quantum theory, the concept of building bricks loses its sense. Fields of tangent Minkowski spacetime are defined by particles; the Poincaré group describes, but does not explain the particle spectrum, i.e., their actual mass, spin (polarization) and electromagnetic charge. With the harmonic flat space analysis and a perturbation expansion, appropriate for scattering theory, there goes a decomposition of a dynamics (Lagrangian) into free particle contribution (kinetic terms, on-shell) and interaction (off-shell). For nonscattering states, such a decomposition, related to classical equations of motion, does not make sense. Spacetimes, in general, cannot be implemented and harmonically analyzed by particles and their Feynman propagators, i.e., by Hilbert spaces with representations of tangent flat Minkowski spacetime, characterized by the two invariants for translations (mass) and rotations (spin, polarization). It is proposed that, in some analogy to the identification of interaction and nonflat geometry (curvature) in general relativity, nonabelian motion and isotropy groups of spacetime - especially with nonflat position - parametrize the basically relevant operations whose representations determine interactions and particle properties. The invariants of the non-Minkowski spacetime representations can explain the particle properties; the tangent Lie algebra structure leads to Minkowski spacetime and free particles. The representation normalizations will be related to coupling constants like Sommerfeld's constant in the residue of the energy-momentum propagator $\frac{\alpha_{\text {Som }}}{q^{2}}$ with a massless pole for electromagnetism. 
The negative energy (bound) state adequate hyperbolic Kepler position with invariant $\overrightarrow{\mathbf{p}}^{2}-\frac{2}{\mathbf{r}}=2 \mathbf{H}$ and the related $\mathbf{S L}(2, \mathbb{C})$-representations with the characteristic dipole measure $e^{-|B| r}=\int \frac{d^{3} q}{\pi^{2}} \frac{|B|}{\left(\vec{q}^{2}+B^{2}\right)^{2}} e^{i \vec{q} \vec{x}} \in L^{\infty}\left(\mathcal{Y}^{3}\right)_{+}, \mathcal{Y}^{3} \cong \mathbb{R}^{3},\left(\vec{\partial}^{2}+B^{2}\right)^{2} e^{-|B| r}=8 \pi|B| \delta(\vec{x})$, for the cyclic hyperbolic ground state is embeddable in spacetime. Its extension by the noncompact abelian causal group $\mathbf{D}(1) \cong \mathbb{R}$ for dilations and (eigen)time translations and representations with energy poles like $\cos E t=\int d q \epsilon(q) q \delta\left(q^{2}-E^{2}\right) e^{i q t} \in L^{\infty}(\mathbb{R})_{+}$, $\left(-d_{t}^{2}-E^{2}\right) \cos E t=0$, for the cyclic spherical ground state defines electroweak spacetime $\mathbb{D}(2)=\mathbf{D}(1) \times \mathcal{Y}^{3}$. As unitary relativity $\mathbb{D}(2) \cong \mathbf{G L}(2, \mathbb{C}) / \mathbf{U}(2)$, it parametrizes the classes of internal hyper-isospin operations $\mathbf{U}(2)$ in the dilation- and phase-extended Lorentz group $\mathbf{G L}(2, \mathbb{C})=\mathbf{D}(1) \times \mathbf{U}(1) \circ \mathbf{S L}(2, \mathbb{C})$ (hyper-Lorentz group), familiar as homogeneous action groups in the electroweak standard model. In a fibration, $\mathbf{G L}(2, \mathbb{C})$ can be considered as a coset bundle with noncompact electroweak spacetime $\mathbb{D}(2)$ as base manifold and compact hyperisospin $\mathbf{U}(2)$ as typical fiber. Its representations are proposed to determine the basic interactions and the particle spectrum.

The representations of hyperbolic Kepler position $\mathcal{Y}^{3} \cong \mathbf{S L}(2, \mathbb{C}) / \mathbf{S U}(2)$ display the noncompact-compact reciprocity $\frac{1}{B^{2}}=(1+2 J)^{2}$ for the (binding) energies $2 E=\vec{q}^{2}=$ $(i B)^{2}$ of the atoms, which determines "nonrelativistic kinetic energies with imaginary momenta," i.e., the hyperboloid curvature, related to the noncompact hyperbolic motion group $\mathbf{S L}(2, \mathbb{C}) \supset \mathbf{S O}_{0}(1,1)$ with continuous invariants, by the principal quantum number $1+2 J=1,2, \ldots(\mathbf{S U}(2)$-multiplicities as Plancherel measure), related to the compact group $\mathbf{S U}(2) \supset \mathbf{S O}(2)$ with discrete invariants. The expression of the energy as time $\mathbf{D}(1)$-invariant by position representations, $2 \mathbf{H}=\mathbf{p}^{2}+2 V(\mathbf{x})$, as in nonrelativistic quantum mechanics, is no longer possible for relativistic spacetimes. Correspondingly, there is no naive takeover of the Schrödinger equation. For electroweak spacetime, the time $\mathbf{D}(1)$ and the position $\mathcal{Y}^{3}$-representation structures are "embedded" in $\mathbb{D}(2)$-representations and a noncompact-compact reciprocity where the two continuous invariants $\left(\mathrm{m}^{2}, M^{2}\right)$ for the maximal noncompact abelian motion group $\mathbf{D}(1) \times \mathbf{S O}_{0}(1,1) \subset \mathbf{G L}(2, \mathbb{C})$ come in the logarithm of their ratio $\int_{m^{2}}^{M^{2}} \frac{d \kappa^{2}}{\kappa^{2}}=\log \frac{M^{2}}{m^{2}}$.

In contrast to the inverse quadratically spaced energy levels in the atomic spectrum $2 E=$ $-\frac{m_{R} c^{2}}{(1+2 J)^{2}} \in\left[-m_{R} c^{2}, 0\left[\right.\right.$ with the Rydberg energy $m_{R} c^{2} \sim 27.2 \mathrm{eV}$ as unit, the range of experimentally relevant masses for particle physics is rather large, even for logarithmic ratios $(\log 10 \sim 2.3$ ) which are characteristic for even-dimensional spacetimes:

\begin{tabular}{lllc}
\hline mass $m_{1}$ & $\frac{m_{1}}{m_{2}}$ & decimal & $\log \frac{m_{1}^{2}}{m_{2}^{2}}$ \\
\hline electron $m_{e} \sim 0.5 \mathrm{MeV}$ & $\frac{m_{e}}{m_{R}}=\frac{1}{\alpha_{\text {Som }}^{2}}$ & $1.9 \times 10^{4}$ & 19.6 \\
proton $m_{p} \sim 938 \mathrm{MeV}$ & $\frac{m_{p}}{m_{e}}$ & $1.8 \times 10^{3}$ & 15 \\
weak boson $m_{W} \sim 80.4 \mathrm{GeV}$ & $\frac{m_{W}}{m_{p}}$ & $0.8 \times 10^{2}$ & 8.8 \\
Planck mass $m_{P} \sim 1.2 \times 10^{19} \mathrm{GeV}$ & $\frac{m_{P}}{m_{W}}$ & $4.5 \times 10^{17}$ & 81 \\
\hline
\end{tabular}

Similar to operational positions with global motion and local isotropy groups, there are distinguished operational spacetimes. Minkowski spacetime, implemented by free particles, cannot explain the properties of interactions and particles. The representations and invariants of a distinguished operational spacetime with a nonflat position are candidates for the determination of the particle spectrum. 
Einstein's relativity and gravity in $(n=4)$-dimensional spacetime involves a dynamical theory of dilation representations: In addition to maximally $\left(\begin{array}{l}n \\ 2\end{array}\right)=6$ independent compact rotation degrees of freedom, the $\left(\begin{array}{c}n+1 \\ 2\end{array}\right)=10$ components of the symmetric spacetime metrical tensor $\mathrm{g}$ contain maximally $n=4$ independent dilation degrees of freedom for the lengths (units) of the $n=4$ principal axes of the metrical hyperboloid. This is visible in the form $\mathbf{g}_{i j}=\mathbf{e}_{i}^{k} \eta_{k l} \mathbf{e}_{j}^{l}$, diagonalized to an orthonormal $\mathbf{O}(t, s)$-form with $t+s=n$ by an $n$-bein (tetrad) $\mathbf{e}(x)=\mathbf{O}(x) \circ \mathbf{d}(x) \in \mathbf{G L}(n, \mathbb{R})$, involving the maximal compact subgroup from the Iwasawa and Cartan factorizations of the structure group for the bases, explicitly for $n=1+3$ :

$$
\begin{aligned}
& \mathbf{G L}(n, \mathbb{R})=\mathbf{O}(n) \circ \mathbf{D}(1) \circ \mathbf{S O}_{0}(1,1)^{n-1} \circ \exp \mathbb{R}^{\left(\begin{array}{c}
n \\
2
\end{array}\right)} \\
&= \mathbf{O}(n) \circ \mathbf{D}(1) \circ \mathbf{S O}_{0}(1,1)^{n-1} \circ \mathbf{O}(n), \\
& \mathbf{g}=\mathbf{O} \circ \operatorname{diag} \mathbf{g} \circ \mathbf{O}^{T}=\mathbf{O} \circ \boldsymbol{d} \circ \eta \circ \boldsymbol{d}^{T} \circ \mathbf{O}^{T}=\mathbf{e} \circ \eta \circ \mathbf{e}^{T} \\
& \text { with } \quad \mathbf{O}(x) \in \mathbf{O}(4), \operatorname{diag} \mathbf{g}=\boldsymbol{d} \circ \eta \circ \boldsymbol{d}^{T} \\
&\left.\eta \begin{array}{llll}
\psi^{\psi_{0}(x)} & 0 & 0 & 0 \\
0 & e^{\psi_{1}(x)} & 0 & 0 \\
0 & 0 & e^{\psi_{2}(x)} & 0 \\
0 & 0 & 0 & e^{\psi_{3}(x)}
\end{array}\right) \in \mathbf{D}(1) \times \mathbf{S O}_{0}(1,1)^{3} .
\end{aligned}
$$

The $n$-bein (moving basis) parametrizes the manifold $\mathbf{G L}(n, \mathbb{R}) / \varnothing(t, s)$ for classes of tangent space bases with $n^{2}-\left(\begin{array}{c}n \\ 2\end{array}\right)=\left(\begin{array}{c}n+1 \\ 2\end{array}\right)=\left(\begin{array}{l}n \\ 2\end{array}\right)+n=10$ real dimensions. The metric and the tetrad which embed the potentials of non-relativistic theories are dilation representation functions (distributions). In Einstein's equations, their derivations $\partial \partial \mathbf{g} \sim \partial \partial \mathbf{e} \sim$ $(\partial \partial \mathbf{O}, \partial \partial \mathbf{d})$, i.e., the representations of the generators of the metrical rotations and dilations in the spacetime curvature are determined by the energy-momentum tensor, which, in flat spacetime, gives the $n=4$ translation currents. Translations can be considered to be dilation generators, $\mathbb{R}=\log \mathbf{D}(1)$.

A position motion group $\mathbf{S O}(3)$ for spherical symmetry leaves maximally two independent dilations $\left(\begin{array}{ll}e^{\psi_{0}} & 0 \\ 0 & e^{|\vec{\psi}|} \mathbf{1}_{3}\end{array}\right)$ - for time and position. A famous example is the Schwarzschild metric with the Schwarzschild length $\ell=\frac{G M}{c^{2}}$ as sole unit for a rotation parabolic position with static dilation properties outside the horizon embedding the Newton potential $-\vec{\partial}^{2} \frac{\ell}{r}=\ell 4 \pi \delta(\vec{x})-$ in the Cartesian parametrization:

$$
\begin{aligned}
& \mathbf{e}(x)=\mathbf{d}(r) \cong\left(\begin{array}{r}
1-\frac{\frac{\ell}{r}}{1+\frac{\ell}{r}} 0 \\
0 \quad \\
\left(1+\frac{\ell}{r}\right)^{2} \mathbf{1}_{3}
\end{array}\right) \in \mathbf{D}(1) \times \mathbf{S O}_{0}(1,1) \text { for }|\vec{x}|=r>\ell, \\
& e^{\psi_{0}(r)}=\frac{1-\frac{\ell}{r}}{1+\frac{\ell}{r}}=1-\frac{2 \ell}{r}+\ldots, \tanh \frac{\psi_{0}(r)}{2}=-\frac{\ell}{r}, \\
& e^{|\vec{\psi}|(r)}=\left(1+\frac{\ell}{r}\right)^{2}=1+\frac{2 \ell}{r}+\ldots, \tanh \frac{|\vec{\psi}|(r)}{4}=\frac{\frac{\ell}{2 r}}{1+\frac{\ell}{2 r}} .
\end{aligned}
$$

Position ("space") is embedded in spacetime. Friedman universes $\mathbb{M}$ display the time development of the three maximally symmetric (conformally Euclidean) operational positions $\mathbb{M} \ni\left(\Omega^{3}, \mathbb{E}^{3}, \mathcal{Y}^{3}\right)$ for the rotation group classes with definite metric 
$d s_{Q^{2}}^{2}=\frac{d \vec{x}^{2}}{\left(1-\frac{Q^{2} \vec{x}^{2}}{4}\right)^{2}}$ and position invariants (units) $Q^{2}=\left(-P^{2}, 0, B^{2}\right)$ in the local dilation factors. They are used for cosmological spacetime models with Robertson-Walker metrical tensors,

$$
\mathbf{g}=d t^{2}-\rho^{2}(t) d s_{Q^{2}}^{2}, \text { with } \mathbf{e}_{Q^{2}}(x)=\mathbf{d}_{Q^{2}}(t, r) \cong\left(\begin{array}{ll}
1 & 0 \\
0 & \frac{\rho(t)}{1-\frac{Q^{2} r^{2}}{4}} \mathbf{1}_{3}
\end{array}\right) \in \mathbf{D}(1),
$$

which involve, as position expansion factor ("radius"), a time representation coefficient $t \longmapsto \rho(t)$ with a time invariant (unit). A trivial time representation $\rho=1$ characterizes non-relativistic space-times $\mathbb{R} \times \mathbb{M}$, e.g., non-relativistic Kepler space-time $\mathbb{R} \times \mathcal{Y}^{3}$ as represented by the non-relativistic hydrogen atom. In general, Friedman universes have two invariants - a time and a position unit.

The three maximally symmetric spacetimes with (1,3)-signature metric parametrize classes of the noncompact Lorentz group (local symmetry or local equivalence classes). They have 10-dimensional motion groups (global symmetry) - the de Sitter group $\mathbf{S O}_{0}(1,4)$, the Poincaré group $\mathbf{S O}_{0}(1,3) \overrightarrow{\times} \mathbb{R}^{4}$, and the anti-de Sitter group $\mathbf{S O}_{0}(2,3)$ which are the compact, flat and noncompact $\mathbf{S O}_{0}(1,3)$-expansions, respectively. Implementing the noncompact and compact subgroups $\mathbf{S O}_{0}(1,1), \mathbb{R}$, and $\mathbf{S O}(2)$, the "radius" grows or shrinks exponentially, stays constant, or oscillates, respectively, with time:

de Sitter spacetime: $\rho(t) \sim e^{ \pm b t}, \quad \mathbf{S O}_{0}(1,4) / \mathbf{S O}_{0}(1,3) \cong \mathcal{Y}^{(1,3)}$,

Minkowski spacetime: $\rho(t) \sim 1, \quad \mathbf{S O}_{0}(1,3) \overrightarrow{\times} \mathbb{R}^{4} / \mathbf{S O}_{0}(1,3) \cong \mathbb{R}^{(1,3)}$, anti-de Sitter spacetime: $\rho(t) \sim e^{ \pm i p t}, \quad \mathbf{S O}_{0}(2,3) / \mathbf{S O}_{0}(1,3) \cong \mathcal{Y}^{(3,1)}$.

Both the de Sitter and anti-de Sitter spacetime are a one-shell hyperboloid, spacelike $\mathcal{Y}^{(1,3)}$ and timelike $\mathcal{Y}^{(3,1)}$. The conformally Minkowskian metrical tensors can be parametrized by a flat metric with one overall dilation factor and one spacetime invariant (unit):

$$
\mathbf{g}=\left(\frac{d x^{2}}{\left(1+b^{2} x^{2}\right)^{2}}, d x^{2}, \frac{d x^{2}}{\left(1-p^{2} x^{2}\right)^{2}}\right) \cong \frac{d x^{2}}{\left(1-k x^{2}\right)^{2}}, k=(-1,0,1),
$$

with $x^{2}=x_{0}^{2}-\vec{x}^{2}, d x^{2}=d x_{0}^{2}-d \vec{x}^{2}$.

In a complex representation, the two nonflat positions which parametrize $\mathbf{S U}(2)$-classes, i.e., compact spherical Einstein position $\Omega^{3} \cong[\mathbf{S U}(2) \times \mathbf{S U}(2)] / \mathbf{S U}(2) \cong \exp (i \mathbb{R})^{3}$, and noncompact hyperbolic Kepler position $\mathcal{Y}^{3} \cong \mathbf{S L}(2, \mathbb{C}) / \mathbf{S U}(2) \cong \exp \mathbb{R}^{3}$, can be supplemented by a corresponding pair with compact time $\mathbf{U}(1)=\exp i \mathbb{R}$ (phase group) and noncompact time $\mathbf{D}(1)=\exp \mathbb{R}$ (dilation group):

$$
\begin{aligned}
& \mathbf{U} U(2) \cong \mathbf{U}(1) \times \Omega^{3} \cong[\mathbf{U}(2) \times \mathbf{U}(2)] / \mathbf{U}(2) \cong \exp (i \mathbb{R})^{4}, \\
& \mathbb{D}(2) \cong \mathbf{D}(1) \times \mathcal{Y}^{3} \cong \mathbf{G L}(2, \mathbb{C}) / \mathbf{U}(2) \cong \exp \mathbb{R}^{4} \text {. }
\end{aligned}
$$

The global motion groups $\mathbf{U}(2) \times \mathbf{U}(2) \cong \exp \left[(i \mathbb{R})^{4} \oplus(i \mathbb{R})^{4}\right]$ and $\mathbf{G L}(2, \mathbb{C}) \cong$ $\exp \left[(i \mathbb{R})^{4} \oplus \mathbb{R}^{4}\right]$, both real eight-dimensional, constitute a compact-noncompact pair. The local compact fixgroup $\mathbf{U}(2) \cong \exp (i \mathbb{R})^{4}$ is real four-dimensional. It is the maximal compact group in the Iwasawa decomposition of the full linear group $\mathbf{G L}(2, \mathbb{C})=\mathbf{U}(2) \circ \mathbf{D}(1) \circ$ $\mathbf{S O}_{0}(1,1) \circ \exp \mathbb{R}^{2}$.

Only the noncompact manifold $\mathbb{D}(2) \cong \mathbf{G L}(2, \mathbb{C}) / \mathbf{U}(2)$ with a hyperbolic Kepler position submanifold comes with the general relativistic nontrivial $(1,3)$-causal structure, compatible with the motion group. It is interpreted as the classes of the hyper-isospin group in the hyper-Lorentz group and called electroweak spacetime. Electroweak spacetime is 
not maximally symmetric. It can be parametrized by the strictly future (positive) subset of Minkowski spacetime which also supports the interaction contributions ("virtual particles") of Feynman propagators. The definition of electroweak spacetime does not involve the real eight-dimensional color group $\mathbf{S U}(3)$ as a constitutive operation group.

\section{Special Spacetime Distributions}

Scalar Feynman distributions with one invariant for possibly indefinite orthogonal groups, implemented by singularities $q^{2}=k=0, \pm 1$ (with units $q^{2}=k m^{2}$ ) in the complex $q^{2}$ plane, give complex argument Macdonald functions $\mathcal{K}$ - where the involved Gamma and Beta functions are defined:

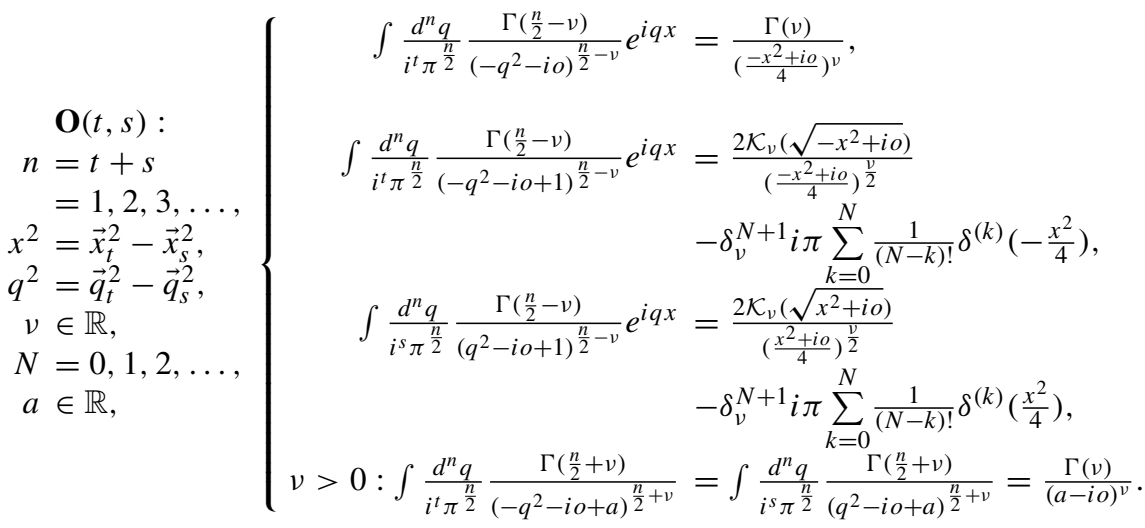

In addition, there occur, for nontrivial signature $s t>0$ and integers $v=N+1=1,2, \ldots$, "lightcone"-supported $\left(x^{2}=0\right)$ Dirac distributions. The corresponding "anti-Feynman" distributions are obtained by canonical conjugation.

In the case of simple poles, especially for the on-shell contributions $\delta\left(q^{2}-k m^{2}\right)$, e.g., of $\mathbf{O}(1,3)$-Feynman propagators, an interpretation of the Fourier transformation with time and position translations and energies and momenta makes sense. Simple poles $\frac{n}{2}-v=1$ are for abelian groups; nonabelian groups use higher order poles $\frac{n}{2}-v=2,3, \ldots$ The invariants at higher order singularities $q^{2}=\mathrm{km}^{2}$ characterize non-translation representations (therefore in the following often the quotation marks in "energies" or "momenta").

The real and imaginary arguments go with "time- and spacelike"-supported contributions, i.e., for opposite definiteness:

$$
|x|=\sqrt{\left|x^{2}\right|}: \sqrt{\mp x^{2}+i o}=\vartheta\left( \pm x^{2}\right) i|x|+\vartheta\left(\mp x^{2}\right)|x| .
$$

The related exponentials give Macdonald and Hankel (with Neumann $\mathcal{N}$ and Bessel $\mathcal{J}$ ) functions, which go with exponential, cosine and sine:

$$
\begin{aligned}
e^{-\sqrt{\mp x^{2}+i o}} & =\vartheta\left( \pm x^{2}\right) e^{-i|x|}+\vartheta\left(\mp x^{2}\right) e^{-|x|}, \\
\text { with } e^{-i|x|} & =\cos |x|-i \sin |x|, \\
\frac{2 \mathcal{K}_{v}\left(\sqrt{\mp x^{2}+i o}\right)}{\left(\frac{\mp x^{2}+i o}{4}\right)^{\frac{v}{2}}} & =\vartheta\left( \pm x^{2}\right) \frac{2 \mathcal{K}_{v}(i|x|)}{\left(i\left|\frac{x}{2}\right|\right)^{v}}+\vartheta\left(\mp x^{2}\right) \frac{2 \mathcal{K}_{v}(|x|)}{\left|\frac{x}{2}\right|^{v}}, \\
\text { with } 2 \mathcal{K}_{v}(i|x|) & =-\pi \mathcal{N}_{v}(|x|)-i \pi \mathcal{J}_{v}(|x|) .
\end{aligned}
$$


Here, the decomposition into "time- and spacelike" contribution is different from the realimaginary decomposition.

For scalar integrals, 2-sphere spreads $\pm \frac{\partial}{\partial \frac{x^{2}}{4 \pi}}$ produce, in steps of two for the dimensions and in steps of one for the orthogonal Lie algebra ranks, higher-dimensional integrals, with the somewhat formal Fourier integral-relations:

$$
\begin{aligned}
& \mathbf{O}(t, s) \rightarrow \mathbf{O}\left(t-2 R_{t}, s-2 R_{s}\right): 2 R_{t}<t, 2 R_{s}<s, \\
& R_{t}+R_{s}=0,1, \ldots: \int d^{n} q \mu\left(q^{2}\right) e^{i q x}=(-1)^{R_{s}}\left(\frac{\partial}{\frac{\partial x^{2}}{4 \pi}}\right)^{R_{t}+R_{s}} \int d^{n-2\left(R_{t}+R_{s}\right)} q \mu\left(q^{2}\right) e^{i q x},
\end{aligned}
$$

for example, scalar Euclidean integrals in odd dimensions and scalar Minkowskian integrals in even dimensions:

$$
\begin{aligned}
& \mathbf{O}\left(2 R_{s}+1\right) \rightarrow \mathbf{O}(1): \int \frac{d^{2 R_{s}+1} q}{\pi^{R_{s}+1}} \mu\left(\vec{q}^{2}\right) e^{i \vec{q} \vec{x}}=\left(-\frac{\partial}{\partial \frac{\vec{x}^{2}}{4}}\right)^{R_{s}} \int \frac{d q}{\pi} \mu\left(q^{2}\right) e^{i q|\vec{x}|}, \\
& \mathbf{O}(1,2 R-1) \rightarrow \mathbf{O}(1,1): \int \frac{d^{2 R} q}{\pi^{R}} \mu\left(q^{2}\right) e^{i q x}=\left(-\frac{\partial}{\partial \frac{x^{2}}{4}}\right)^{R-1} \int \frac{d^{2} q}{\pi} \mu\left(q^{2}\right) e^{i\left(q_{0} x_{0}-q_{3}|\vec{x}|\right)} .
\end{aligned}
$$

Nontrivial $\mathbf{O}(t, s)$-properties with $L=0,1,2, \ldots$ are effected by harmonic "(energy)momentum" polynomials $(q)^{L}$ and derivatives $\left(\frac{\partial}{\partial q}\right)^{L}=(2 q)^{L}\left(\frac{\partial}{\partial q^{2}}\right)^{L}$.

\section{Maximally Symmetric Spacetimes}

The three maximally symmetric signature $(t, s)$-manifolds with dimension $n=t+s=$ $1,2, \ldots$ are conformally flat. They are characterized by one spacetime invariant (unit) and have, for $n \geq 2$, constant curvature - two general hyperboloids and a flat space:

$$
\left.\begin{array}{l}
\mathcal{Y}^{(t, s)} \cong \mathbf{S O}_{0}(t, 1+s) / \mathbf{S O}_{0}(t, s), \quad k=-1, \\
\mathbb{R}^{(t, s)} \cong \mathbf{S O}_{0}(t, s) \overrightarrow{\times} \mathbb{R}^{n} / \mathbf{S O}_{0}(t, s), k=0, \\
\mathcal{Y}^{(s, t)} \cong \mathbf{S O}_{0}(1+t, s) / \mathbf{S O}_{0}(t, s), \quad k=1,
\end{array}\right\} \begin{array}{r}
\mathbf{g}=\frac{d x^{2}}{\left(1+k m^{2} x^{2}\right)^{2}}, \\
x^{2}=\vec{x}_{t}^{2}-\vec{x}_{s}^{2},
\end{array}
$$

with time $\mathbb{R}^{(1,0)}$, the Euclidean spaces $\mathbb{E}^{s}=\mathbb{R}^{(0, s)}$, Minkowski spacetime $\mathbb{R}^{(1,3)}$, the spheres $\Omega^{s}=\mathcal{Y}^{(0, s)}$, the causal hyperboloids $\mathcal{Y}^{s}=\mathcal{Y}^{(s, 0)}$, and the (anti-)de Sitter universes $\mathcal{Y}^{(1,3)}$ and $\mathcal{Y}^{(3,1)}$.

The relevant representation coefficients for the flat spaces (translations) use Dirac (energy-)momentum distributions which are related to general Feynman propagators with simple poles, characteristic for abelian groups:

$$
\mathbb{R}^{(t, s)}: \int d^{n} q \delta\left(-q^{2}+k m^{2}\right) e^{i q x} \text { from } \int \frac{d^{n} q}{i \pi} \frac{1}{-q^{2}-i o+k m^{2}} e^{i q x},
$$

e.g., for time $\delta\left(-q_{0}^{2}+E^{2}\right)$ with energies, and for Euclidean position $\delta\left(\vec{q}^{2}-P^{2}\right)$ with momenta. All unitary Poincaré group $\mathbf{S L}(2, \mathbb{C}) \overrightarrow{\times} \mathbb{R}^{4}$-representations with nontrivial translations are induced by unitary representations of the direct product subgroups $\mathbf{S U}(2) \times \mathbb{R}^{4}$, $\mathbf{S O}(2) \times \mathbb{R}^{4}$ and $\mathbf{S U}(1,1) \times \mathbb{R}^{4}$ for $k=1,0,-1$, respectively. Only causal spacetime invariants $k=1$, i.e., $\mathrm{km}^{2} \geq 0$, are used as masses for, respectively, massive and massless particles (more below).

For odd dimensions $n=1+2 R=1,3, \ldots$, e.g., for one-dimensional time and three-dimensional positions, the relevant scalar coefficients for $\mathcal{Y}^{(t, s)}$ and $\mathcal{Y}^{(s, t)}$ for cyclic 
(ground) states and - for $n \geq 3-$ interactions involve half-integer index functions. The corresponding functions and distributions have one invariant $m^{2}>0$ (or analogously with $m^{2}<0$ ) in the exponentials - as a spherical or a hyperbolic invariant for "time- and spacelike" support, respectively:

$$
\begin{aligned}
\int \frac{d^{1+2 R} q}{i^{t} \pi^{1+R}} \frac{\Gamma(1+R)}{\left(-q^{2}-i o+m^{2}\right)^{1+R}} e^{i q x} & =\frac{e^{-\sqrt{-m^{2} x^{2}+i o}}}{|m|}=\vartheta\left(x^{2}\right) \frac{e^{-i|m x|}}{|m|}+\vartheta\left(-x^{2}\right) \frac{e^{-|m x|}}{|m|}, \\
\int \frac{d^{1+2 R} q}{i^{t} \pi^{1+R}} \frac{\Gamma(R)}{\left(-q^{2}-i o+m^{2}\right)^{R}} e^{i q x} & =\frac{e^{-\sqrt{-m^{2} x^{2}+i o}}}{\frac{\sqrt{-x^{2}+i o}}{4}}=\vartheta\left(x^{2}\right) \frac{e^{-i|m x|}}{i \frac{x \mid}{2}}+\vartheta\left(-x^{2}\right) \frac{e^{-|m x|}}{\frac{|x|}{2}} .
\end{aligned}
$$

The relevant scalar integer index functions for even dimensions $n=2 R=2,4, \ldots$, e.g., for (anti-)de Sitter spacetimes $\mathcal{Y}^{(1,3)}$ and $\mathcal{Y}^{(3,1)}$, integrate - with a positive measure - the exponentials for odd dimensions over an invariant line, i.e., $\frac{e^{-\ell|\kappa x|}}{|\kappa|}$ for states and $\frac{e^{-\ell|\kappa x|}}{\frac{|x|}{2}}$ for interactions with compactness $\iota=(1, i)$ :

$$
\begin{aligned}
\int \frac{d^{2 R} q}{i^{t} \pi^{R}} \frac{\Gamma(1+R)}{\left(-q^{2}-i o+m^{2}\right)^{1+R}} e^{i q x} & =\int_{m^{2}}^{\infty} \frac{d \kappa^{2}}{2 \sqrt{\left(\kappa^{2}-m^{2}\right)^{3}}} \frac{e^{-\sqrt{-\kappa^{2} x^{2}+i o}}}{|\kappa|} \\
\int \frac{d^{2 R} q}{i^{t} \pi^{R}} \frac{\Gamma(R)}{\left(-q^{2}-i o+m^{2}\right)^{R}} e^{i q x} & =\int_{m^{2}}^{\infty} \frac{d \kappa^{2}}{2 \sqrt{\left(\kappa^{2}-m^{2}\right)^{3}}} \frac{e^{-\sqrt{-\kappa^{2} x^{2}+i o}}}{\sqrt{\frac{-x^{2}+i o}{4}}} .
\end{aligned}
$$

The residual normalization takes into account the pairwise rank structure for orthogonal operations (more ahead). For odd-dimensional spaces, the residual integration $\frac{d^{1+2 R} q}{\pi^{1+R}}$ is used for a "point-residue," and $\frac{d^{2 R} q}{\pi^{R}}$ for even-dimensional spaces, leaving a dilation $\kappa^{2}$ integration for a "line-residue".

\section{Minkowski Spacetime}

Canonical quantum field theory works with particle fields. They represent the Poincaré group as motion group of Minkowski spacetime and are also used for an expansion of interactions.

The real 10-dimensional Lie algebra of the Poincaré group $\mathbf{S L}(2, \mathbb{C}) \overrightarrow{\times} \mathbb{R}^{4} \sim$ $\mathbf{S O}_{0}(1,3) \overrightarrow{\times} \mathbb{R}^{4}$ for relativistic scattering is spanned by angular momenta and boosts $\mathbf{L}^{i k} \sim$ $\left(\mathbf{J}^{a}, \mathbf{B}^{a}\right)$ and the energy-momenta $\mathbf{p}^{i}$ with the Lie brackets:

$$
\begin{aligned}
{\left[\mathbf{L}^{i k} \mathbf{L}^{n m}\right] } & =\eta^{i n} \mathbf{L}^{k m}-\eta^{k n} \mathbf{L}^{i m}-\eta^{i m} \mathbf{L}^{k n}+\eta^{k m} \mathbf{L}^{i n}, \\
{\left[\mathbf{L}^{i k} \mathbf{p}^{n}\right] } & =\eta^{i n} \mathbf{p}^{k}-\eta^{k n} \mathbf{p}^{i},\left[\mathbf{p}^{i} \mathbf{p}^{n}\right]=0,
\end{aligned}\left\{\begin{array}{l}
{\left[\mathbf{J}^{a}, \mathbf{p}^{0}\right]=0,} \\
{\left[\mathbf{J}^{a}, \mathbf{p}^{b}\right]=-\epsilon^{a b c} \mathbf{p}^{c},} \\
{\left[\mathbf{B}^{a}, \mathbf{p}^{0}\right]=\mathbf{p}^{a},} \\
{\left[\mathbf{B}^{a}, \mathbf{p}^{b}\right]=\delta^{a b} \mathbf{p}^{0} .}
\end{array}\right.
$$

The Poincaré Lie algebra has two independent invariants, first the squared energy-momenta $\mathbf{p}^{2}=\eta_{i k} \mathbf{p}^{i} \mathbf{p}^{k}$ with continuous spectrum for the Hilbert representations:

$$
\left[\mathbf{L}^{i k}, \mathbf{p}^{2}\right]=0,\left[\mathbf{p}^{i}, \mathbf{p}^{2}\right]=0, \mathbf{s p e c}_{+} \mathbf{p}^{2}=\left\{m^{2} \in \mathbb{R}\right\} .
$$

The second invariant uses the Lubanski-Pauli vector $\boldsymbol{S}^{i}$, given by the Lorentz group generators, projected on the energy-momenta with the embedded momentum projection of the 
angular momenta as zeroth component, supplemented by the rest-system spin generator (with $\mathbf{p} \Rightarrow\left(\begin{array}{c}m \\ 0\end{array}\right)$ ):

$$
\begin{aligned}
& \mathbf{S}_{m}=\frac{1}{2} \epsilon_{i k n m} \mathbf{L}^{i k} \mathbf{p}^{n} \text { with }\left(\begin{array}{l}
\mathbf{S}_{0} \\
\overrightarrow{\mathbf{S}}
\end{array}\right) \cong\left(\begin{array}{l}
\overrightarrow{\mathbf{J}} \overrightarrow{\mathbf{p}} \\
\overrightarrow{\mathbf{J}} \mathbf{p}^{0}-\overrightarrow{\mathbf{B}} \times \overrightarrow{\mathbf{p}}
\end{array}\right) \Rightarrow m\left(\begin{array}{l}
0 \\
\overrightarrow{\mathbf{J}}
\end{array}\right), \mathbf{S}_{m} \mathbf{p}^{m}=0, \\
& {\left[\mathbf{L}^{i k} \mathbf{S}^{n}\right]=\eta^{i n} \mathbf{S}^{k}-\eta^{k n} \mathbf{S}^{i},\left[\mathbf{p}^{i}, \mathbf{S}^{n}\right]=0,\left[\mathbf{S}^{i}, \mathbf{S}^{n}\right]=\epsilon^{i n k m} \mathbf{S}_{k} \mathbf{p}_{m} .}
\end{aligned}
$$

The spin invariant is of order four in the Lie algebra elements:

$$
\begin{aligned}
& \mathbf{S}^{2}=\eta^{i k} \mathbf{S}_{i} \mathbf{S}_{k} \cong-\overrightarrow{\mathbf{J}}^{2} \mathbf{p}_{0}^{2}+(\overrightarrow{\mathbf{J}} \overrightarrow{\mathbf{p}})^{2}-\overrightarrow{\mathbf{B}}^{2} \overrightarrow{\mathbf{p}}^{2}+(\overrightarrow{\mathbf{B}} \overrightarrow{\mathbf{p}})^{2}+2(\overrightarrow{\mathbf{J}} \times \overrightarrow{\mathbf{B}}) \mathbf{p}_{0} \overrightarrow{\mathbf{p}} \Rightarrow-m^{2} \overrightarrow{\mathbf{J}}^{2}, \\
& {\left[\mathbf{L}^{i k}, \mathbf{S}^{2}\right]=0,\left[\mathbf{p}^{i}, \mathbf{S}^{2}\right]=0 .}
\end{aligned}
$$

For positive translation invariant (nonvanishing mass), its normalized Hilbert representation spectrum is the familiar spin spectrum:

$$
m^{2}>0: \mathbf{s p e c}_{+}\left(-\frac{\mathbf{S}^{2}}{\mathbf{p}^{2}}\right)=\{-J(J+1) \mid 2 J \in \mathbb{N}\} .
$$

The simple energy-momentum pole Feynman propagators of a neutral scalar field $\boldsymbol{\Phi}$, or of a Dirac field $\Psi$ for idealized ${ }^{2}$ particles with spin $\frac{1}{2}$ (Dirac matrices $\gamma_{j}$ ), and isospin $I \in\left\{0, \frac{1}{2}, 1, \ldots\right\}$ (unit matrix $\mathbf{1}_{1+2 I}$ ), and normalization factors $\rho\left(m^{2}\right)>0$,

$$
\begin{aligned}
\langle 0 \boldsymbol{\Phi}(y) \boldsymbol{\Phi}(x)\rangle 0_{\text {Feynman }} & =\rho\left(m^{2}\right) \int \frac{d^{4} q}{i \pi(2 \pi)^{3}} \frac{1}{-q^{2}-i o+m^{2}} e^{i q(x-y)}, \\
\langle 0 \boldsymbol{\Psi}(y) \overline{\boldsymbol{\Psi}}(x)\rangle 0_{\text {Feynman }} & =\rho\left(m^{2}\right) \int \frac{d^{4} q}{i \pi(2 \pi)^{3}} \frac{\left(\gamma q+\mathbf{1}_{4} m\right) \times \mathbf{1}_{1+2 I}}{-q^{2}-i o+m^{2}} e^{i q(x-y)},
\end{aligned}
$$

decompose into free particle and interaction contribution. The real on-shell $q^{2}=m^{2}$ contribution (particles) from the positive Dirac distribution

$$
\frac{1}{i \pi} \frac{1}{-q^{2}-i o+m^{2}}=\delta\left(q^{2}-m^{2}\right)+\frac{1}{i \pi} \frac{1}{-q_{\mathrm{P}}^{2}+m^{2}},
$$

is the expectation value $\langle 0 \ldots\rangle 0$ of the anticommutator for the Bose field $\Phi$ and of the commutator for the Fermi field $\Psi$ in the Fock ground-state vector $|0\rangle$ for translation coefficients. It is an induced representation matrix element of the Poincaré group, for a scalar field representing nontrivially the translations with the momenta from a hyperboloid $\mathbf{S O}_{0}(1,3) / \mathbf{S O}(3)$ :

$$
\begin{aligned}
& \langle 0|\{\boldsymbol{\Phi}(y) \boldsymbol{\Phi}(x)\}| 0\rangle=\rho\left(m^{2}\right) \int \frac{d^{4} q}{(2 \pi)^{3}} 2 \delta\left(q^{2}-m^{2}\right) e^{i q(x-y)}, \\
& \mathbb{R}^{(1,3)} \ni x \longmapsto \int \frac{d^{4} q}{\pi} \delta\left(q^{2}-m^{2}\right) e^{i q x}=m^{2} \frac{\vartheta\left(x^{2}\right) \pi \mathcal{N}_{1}(|m x|)+\vartheta\left(-x^{2}\right) 2 \mathcal{K}_{1}(|m x|)}{\left|\frac{m x}{2}\right|} .
\end{aligned}
$$

The imaginary off-shell contribution with the principal value simple pole is not a coefficient of a Poincaré group representation, therefore the name "virtual particles." It is a distribution

\footnotetext{
${ }^{2}$ So far, no mass-degenerate nontrivial isospin multiplet particles have been found — in contrast to a massdegeneracy for spin.
} 
of an $\mathbf{O}(1,1)$-coefficient, used for $\mathbf{O}(1,3)$-interactions and only causally supported, not by all translations:

$$
\begin{aligned}
-\epsilon\left(x_{0}-y_{0}\right)\langle 0[\boldsymbol{\Phi}(y), \boldsymbol{\Phi}(x)] \mid 0\rangle & =\rho\left(m^{2}\right) \int \frac{d^{4} q}{i \pi(2 \pi)^{3}} \frac{2}{-q_{\mathrm{P}}^{2}+m^{2}} e^{i q(x-y)} \\
& =0 \text { for }(x-y)^{2}<0, \\
\mathbb{R}_{+}^{4} \cup \mathbb{R}_{-}^{4} \ni x \longmapsto \int \frac{d^{4} q}{i \pi^{2}} \frac{1}{-q_{\mathrm{P}}^{2}+m^{2}} e^{i q x} & =-\epsilon\left(x_{0}\right) \int \frac{d^{4} q}{\pi} \epsilon\left(q_{0}\right) \delta\left(q^{2}-m^{2}\right) e^{i q x} \\
& =i\left[m^{2} \vartheta\left(x^{2}\right) \frac{\pi \mathcal{J}_{1}(|m x|)}{\left|\frac{m x}{2}\right|}-\delta\left(\frac{x^{2}}{4 \pi}\right)\right] .
\end{aligned}
$$

It arises by causal ordering, via $\epsilon\left(x_{0}-y_{0}\right)$, from the on-shell quantization of the free scalar field via the commutator:

$$
\begin{aligned}
{[\boldsymbol{\Phi}(y), \boldsymbol{\Phi}(x)] } & =\mathbf{1} \rho\left(m^{2}\right) \int \frac{d^{4} q}{(2 \pi)^{3}} 2 \epsilon\left(q_{0}\right) \delta\left(q^{2}-m^{2}\right) e^{i q(x-y)} \\
{\left.\left[i \partial^{a} \boldsymbol{\Phi}(y), \boldsymbol{\Phi}(x)\right]\right|_{x_{0}=y_{0}} } & =\mathbf{1} \rho\left(m^{2}\right) \int \frac{d^{4} q}{(2 \pi)^{3}} 2 \epsilon\left(q_{0}\right) q^{a} \delta\left(q^{2}-m^{2}\right) e^{-i \vec{q}(\vec{x}-\vec{y})} \\
& =\mathbf{1} \rho\left(m^{2}\right) 2 \delta_{0}^{a} \delta(\vec{x}-\vec{y})
\end{aligned}
$$

i.e., the off-shell feature is caused by the $\epsilon\left(x_{0}-y_{0}\right)$-multiplication. The causally supported quantization characterizes locality and microcausality. For free fields, the factor $\rho\left(m^{2}\right)>0$, i.e., the residual normalization at the pole $q^{2}=m^{2}$, can be chosen arbitrarily, e.g., $\rho\left(m^{2}\right)=$ 1 or $\rho\left(m^{2}\right)=(2 \pi)^{3}$.

A perturbative Feynman propagator expansion of interactions needs the off-shell propagators: For example, in a Feynman graph for the Compton scattering of an electron $e$ and a photon $\gamma$, described by the gauge vertex $\bar{\Psi}(x) \gamma_{j} \boldsymbol{\Psi}(x) \mathbf{A}^{j}(x)$, the intermediate electron $e^{*}$ cannot be on the mass shell, as seen in the electron's rest system:

$$
e \gamma \rightarrow e^{*} \rightarrow e \gamma:\left\{\begin{array}{c}
q_{e}=(m, 0), q_{\gamma}=(p, p), p \neq 0, \\
\Rightarrow q_{e^{*}}=q_{e}+q_{\gamma}=(m+p, p), \\
q_{e^{*}}^{2}=m^{2}+2 p m \neq m^{2}
\end{array}\right.
$$

Equally, an electron cannot radiate a photon. The virtual electron comes with the principal value part of the electron propagator. The naive off-shell interaction supplements of the on-shell particles are remarkably successful - up to the divergencies. Perturbation theory calculations for relativistic scattering yield, for quantum electrodynamics, one of the most accurate agreements in physics between theory and experiment.

For a particle field propagator, the time projection, effected by position integration, gives as real part from the on-shell (free particle) contribution a harmonic oscillator cosine, i.e., a time representation coefficient with the mass as invariant. The position projection, effected 
by time integration, is nontrivial for the off-shell (interaction) contribution (imaginary part) only. It gives, as position distribution, a Yukawa potential with the inverse mass as its range:

$$
\begin{aligned}
\int d^{3} x \int \frac{d^{4} q}{i \pi(2 \pi)^{3}} \frac{1}{-q^{2}-i o+m^{2}} e^{i q x} & =\int \frac{d q_{0}}{i \pi} \frac{1}{-q_{0}^{2}-i o+m^{2}} e^{i q_{0} x_{0}}=\frac{e^{-i\left|m x_{0}\right|}}{|m|}, \\
\int d^{3} x \int \frac{d^{4} q}{(2 \pi)^{3}} \delta\left(-q^{2}+m^{2}\right) e^{i q x} & =\int d q_{0} \delta\left(-q_{0}^{2}+m^{2}\right) e^{i q_{0} x_{0}}=\frac{\cos m x_{0}}{|m|}, \\
\int d x_{0} \int \frac{d^{4} q}{i \pi(2 \pi)^{3}} \frac{1}{-q^{2}-i o+m^{2}} e^{i q x} & =\int d x_{0} \int \frac{d^{4} q}{i \pi(2 \pi)^{3}} \frac{1}{-q_{\mathrm{P}}^{2}+m^{2}} e^{i q x} \\
& =\int \frac{d^{3} q}{4 i \pi^{3}} \frac{1}{\vec{q}^{2}+m^{2}} e^{-i \vec{q} \vec{x}}=\frac{e^{-|m| r}}{2 \pi i r} .
\end{aligned}
$$

The hyperbolic position dependence of the Yukawa potential comes from the position projection of a causally supported off-shell distribution with $\vartheta\left(x^{2}\right)$, not from a spacelike dependence with $\vartheta\left(-x^{2}\right)$ : Interactions are spacetime-embedded by causally supported distributions of subgroup representations.

The on-shell functions for "real particles" fulfill a homogeneous Klein-Gordon or Dirac equation for the translation invariant of the Poincare group representation $\partial^{2}=-m^{2}$, whereas the off-shell distributions for "virtual particles," i.e., for interactions are introduced and motivated as Green's kernels of the corresponding inhomogeneous equations:

$$
\begin{aligned}
\left(\partial^{2}+m^{2}\right) \int \frac{d^{4} q}{(2 \pi)^{3}}\left(\begin{array}{c}
\delta\left(q^{2}-m^{2}\right) \\
\frac{1}{i \pi} \frac{1}{-q_{\mathrm{P}}^{2}+m^{2}}
\end{array}\right) e^{i q x} & =\left(\begin{array}{l}
0 \\
-2 i \delta(x)
\end{array}\right), \begin{array}{l}
\left(d_{x_{0}}^{2}+m^{2}\right) \cos m x_{0}=0 \\
\left(-\vec{\partial}^{2}+m^{2}\right) \rho \frac{e^{-|m| r}}{4 \pi r}=\rho \delta(\vec{x})
\end{array}, \\
\left(\partial^{2}+m^{2}\right)\langle 0|\boldsymbol{\Phi}(0) \boldsymbol{\Phi}(x)| 0\rangle_{\text {Feynman }} & =-2 i \rho\left(m^{2}\right) \delta(x), \\
\left(i \gamma \partial+\mathbf{1}_{4} m\right)\langle 0|\boldsymbol{\Psi}(0) \bar{\Psi}(x)| 0\rangle_{\text {Feynman }} & =-2 i \rho\left(m^{2}\right) \mathbf{1}_{4} \otimes \mathbf{1}_{1+2 I} \delta(x) .
\end{aligned}
$$

A particle field Feynman propagator has three parts, which can be related to the three maximally symmetric operational positions $\left(\mathbb{E}^{3}, \Omega^{3}, \mathcal{Y}^{3}\right)$ : In addition to the on-shell "flat" contribution with the $(r=0)$-regular spherical Bessel function $\frac{\sin |P| r}{r}$ for the free Euclidean $\mathbb{E}^{3}$-scattering states, there are the causally supported off-shell "non-flat" contributions for energies over the mass threshold with the spherical Neumann function $\frac{\cos P r}{r}$ (spherical Laplacian $\Omega^{3}$-kernel), and for energies under the mass threshold (imaginary momenta) with the Yukawa potential, i.e., the hyperbolic Macdonald function $\frac{e^{-|B| r}}{r}$ (hyperbolic Laplacian $\mathcal{Y}^{3}$-kernel), both singular at $r=0$ :

$$
\begin{gathered}
\int \frac{d^{4} q}{(2 \pi)^{3}}\left(\begin{array}{c}
\delta\left(q^{2}-m^{2}\right) \\
\frac{1}{i \pi} \frac{1}{-q_{\mathrm{P}}^{2}+m^{2}}
\end{array}\right) e^{i q x} \\
=\int \frac{d q_{0}}{(2 \pi)^{2}} e^{i q_{0} x_{0}}\left[\vartheta\left(q_{0}^{2}-m^{2}\right)\left(\begin{array}{c}
\frac{\sin |P| r}{r} \\
\frac{\cos P r}{i r}
\end{array}\right)+\vartheta\left(m^{2}-q_{0}^{2}\right)\left(\begin{array}{c}
0 \\
\frac{e^{-|B| r}}{i r}
\end{array}\right)\right], \\
\text { with }|P|=\sqrt{q_{0}^{2}-m^{2}} \text { and }|B|=\sqrt{m^{2}-q_{0}^{2}}
\end{gathered}
$$


The harmonic expansion of a free scalar field, i.e., its particle analysis, uses distributive momentum-characterized creation and annihilation eigenoperators $\left(\mathrm{u}(\vec{q}), \mathrm{u}^{\star}(\vec{q})\right)$ of the spacetime translations with momenta as eigenvalues in the direct integral decomposition with the invariant hyperbolic measure,

$$
\begin{aligned}
& \mathbb{R}^{(1,3)} \ni x \longmapsto \Phi(x)=\oplus \int \frac{d^{3} q}{q_{0}(2 \pi)^{3}}\left[e^{i q x} \mathrm{u}(\vec{q})+e^{-i q x} \mathrm{u}^{\star}(\vec{q})\right], \\
& d^{4} q 2 \vartheta\left(q_{0}\right) \delta\left(q^{2}-m^{2}\right) \rightarrow \frac{d^{3} q}{q_{0}} \text { with } q_{0}=\sqrt{\vec{q}^{2}+m^{2}} .
\end{aligned}
$$

The harmonic analysis of a Dirac spinor field, e.g., of an electron-positron field, with irreducible left and right Weyl spinor contributions involves translation and rotation eigenoperators for particles $\left(\mathrm{u}^{A}(\vec{q}), \mathrm{u}_{A}^{\star}(\vec{q})\right)$, e.g., negatively charged electrons, $z=-1$, and antiparticles $\left(\mathrm{a}_{A}(\vec{q}), \mathrm{a}^{A \star}(\vec{q})\right)$, then positively charged positrons, $z=+1$, with spin direction (eigenvalue) "up" and "down," $A=1,2$ :

$$
\begin{aligned}
& \boldsymbol{\Psi}(x) \cong\left(\begin{array}{l}
\mathbf{l}^{C} \\
\mathbf{r}^{\dot{C}}
\end{array}\right)(x)=\sqrt{m} \oplus \int \frac{d^{3} q}{q_{0}(2 \pi)^{3}}\left(\begin{array}{c}
s_{A}^{C}\left(\frac{q}{m}\right)\left[e^{i q x} \mathbf{u}^{A}(\vec{q})+e^{-i q x} \mathrm{a}^{\star} A(\vec{q})\right] \\
\hat{s}_{A}^{C}\left(\frac{q}{m}\right)\left[e^{i q x} \mathbf{u}^{A}(\vec{q})-e^{-i q x} \mathrm{a}^{\star} A(\vec{q})\right]
\end{array}\right), \\
& \Psi^{*}(x) \cong\left(\begin{array}{c}
\mathbf{l}_{\dot{C}}^{\star} \\
\mathbf{r}_{C}^{\star}
\end{array}\right)(x)-2 m m=\sqrt{m} \oplus \int \frac{d^{3} q}{q_{0}(2 \pi)^{3}}\left(\begin{array}{c}
s^{-1} \underset{\dot{C}}{\stackrel{A}{C}\left(\frac{q}{m}\right)\left[e^{-i q x} \mathrm{u}_{A}^{\star}(\vec{q})+e^{i q x} \mathrm{a}_{A}(\vec{q})\right]} \\
\hat{s}_{C}^{-1} \underset{C}{\stackrel{q}{m}}\left(\frac{q}{m}\right)\left[e^{-i q x} \mathrm{u}_{A}^{\star}(\vec{q})-e^{i q x} \mathrm{a}_{A}(\vec{q})\right]
\end{array}\right), \\
& \text { with } q_{0}=\sqrt{\vec{q}^{2}+m^{2}} \text {. }
\end{aligned}
$$

$(s, \hat{s})\left(\frac{q}{m}\right)=\sqrt{\frac{q_{0}+m}{2 m}}\left(\mathbf{1}_{2} \pm \frac{\vec{\sigma} \vec{q}}{q_{0}+m}\right)$ are the momentum dependent boost representations (Weyl transmutators), which embed the spin group $\mathbf{S U}(2)$ into the Lorentz group $\mathbf{S L}(2, \mathbb{C})$. The Dirac equation describes this dependence for free massive spin- $\frac{1}{2}$ particles.

The quantization of canonical Bose fields is the position distribution (quantization density) of the quantum mechanical commutator $[i \mathbf{p}, \mathbf{x}]=\mathbf{1}$. The spacetime dependence of canonically quantized free fields is the position distribution of the time translations, $d_{t} \hookrightarrow \partial^{j}$, implemented by harmonic oscillators with

$$
\begin{aligned}
\mathbf{x}(t)=\frac{e^{i|E| t} \mathrm{u}+e^{-i|E| t} \mathrm{u}^{\star}}{\sqrt{2}},\left\{\begin{aligned}
{[\mathbf{x}(s) \mathbf{x}(t)] } & =\mathbf{1} i \sin |E|(t-s) \\
& =|E| \int d q_{0} \epsilon\left(q_{0}\right) \delta\left(q_{0}^{2}-E^{2}\right) e^{i q_{0}(t-s)}, \\
\langle 0|\{\mathbf{x}(s) \mathbf{x}(t)\}| 0\rangle & =\cos E(t-s) \\
& =|E| \int d q_{0} \delta\left(q_{0}^{2}-E^{2}\right) e^{i q_{0}(t-s)} .
\end{aligned}\right. \\
\begin{aligned}
\langle 0|\mathbf{x}(s) \mathbf{x}(t)| 0\rangle_{\text {Feynman }}=\langle 0|\{\mathbf{x}(s) \mathbf{x}(t)\}| 0\rangle-\epsilon(t-s)\langle 0|[\mathbf{x}(s) \mathbf{x}(t)]| 0\rangle
\end{aligned} \\
=\int \frac{d q_{0}}{i \pi} \frac{|E|}{-q_{0}^{2}-i o+E^{2}} e^{i q_{0}(t-s)}=e^{-i|E(t-s)|} .
\end{aligned}
$$

The quantization and Hilbert metric involve the "inverse" of the Lorentz invariant measure $(2 \pi)^{3} q_{0} \delta(\vec{q}-\vec{p}) \leftrightarrow \frac{d^{3} q}{r(2 \pi)^{3} q_{0}}$ for $q_{0}=\sqrt{\vec{q}^{2}+m^{2}}$ of the momentum hyperboloid $\mathcal{Y}^{3}$ :

$$
\begin{aligned}
& \left.\qquad \mathrm{u}^{\star}(\vec{p}) \mathrm{u}(\vec{q})\right]=\mathbf{1} \rho\left(m^{2}\right) q_{0} \delta\left(\frac{\vec{q}-\vec{p}}{2 \pi}\right), \\
& \left\langle 0\left|\left\{\mathrm{u}^{\star}(\vec{p}) \mathrm{u}(\vec{q})\right\}\right| 0\right\rangle=\left\langle 0\left|\mathrm{u}^{\star}(\vec{p}) \mathrm{u}(\vec{q})\right| 0\right\rangle=\left\langle m^{2} ; \vec{p} m^{2} ; \vec{q}\right\rangle=\rho\left(m^{2}\right) q_{0} \delta\left(\frac{\vec{q}-\vec{p}}{2 \pi}\right), \\
& \text { distributive Hilbert space basis: }\left\{\left|m^{2} ; \vec{q}\right\rangle=\mathrm{u}(\vec{q})|0\rangle \mid \vec{q} \in \mathbb{R}^{3}\right\} .
\end{aligned}
$$


The related Hilbert space is constituted by the square integrable functions $L^{2}\left(\mathcal{Y}^{3}\right)$ of the mass shell (momentum hyperboloid) $q^{2}=m^{2}$. The distributive Hilbert space basis for the Dirac field contains eigenvectors for spin $\frac{1}{2}$ particles and antiparticles, e.g., for electrons and positrons with charge numbers $z=\mp 1$ :

$$
\text { for } \Psi:\left\{\begin{array}{l}
\left|m^{2}, \frac{1}{2},-1 ; \vec{q}, A\right\rangle=\mathrm{u}^{A}(\vec{q})|0\rangle, \\
\left|m^{2}, \frac{1}{2},+1 ; \vec{q}, A\right\rangle=\mathrm{a}_{A}(\vec{q})|0\rangle
\end{array} \quad \mid \vec{q} \in \mathbb{R}^{3}, A=1,2\right\}
$$

In this framework, it is by no way obvious how to define the position for a "pointlike" particle and a particle orbit. The quantum mechanical position-momentum "equivalence" does not exist, there is no Heisenberg Lie-algebra. Also, an implementation of the nonrelativistic position-momentum uncertainty, or of an energy-time uncertainty, is by no way straightforward. A Poincare group representation is determined by the invariants mass und spin (polarization) with the momentum and spin (polarization) direction for a possible distributive eigenvalue basis. The quantum algebra for the creation and annihilation operators allows the quantum representations of the Poincare group with the Minkowski translations.

The field action on the Fock ground-state vector gives a cyclic vector $|\mathcal{C}\rangle=\Phi(0)|0\rangle$, summing up all translation "eigenvectors" with spherical waves over the threshold $q_{0} \geq|\mathrm{m}|$ :

$$
\begin{aligned}
& \left.x \bullet|\mathcal{C}\rangle=\boldsymbol{\Phi}(x)|0\rangle=\oplus \int \frac{d^{3} q}{q_{0}(2 \pi)^{3}} e^{i q x} m^{2} ; \vec{q}\right\rangle, \text { with } q_{0}=\sqrt{\vec{q}^{2}+m^{2}}, \\
& \langle\mathcal{C}|x \bullet| \mathcal{C}\rangle=\langle 0|\boldsymbol{\Phi}(0) \boldsymbol{\Phi}(x)| 0\rangle=\rho\left(m^{2}\right) \int \frac{d^{4} q}{(2 \pi)^{3}} 2 \vartheta\left(q_{0}\right) \delta\left(q^{2}-m^{2}\right) e^{i q x}, \\
& \int \frac{d^{4} q}{\pi} 2 \vartheta\left(q_{0}\right) \delta\left(q^{2}-m^{2}\right) e^{i q x}=m^{2} \frac{\vartheta\left(x^{2}\right)\left[\pi \mathcal{N}_{1}(|m x|)-i \epsilon\left(x_{0}\right) \pi \mathcal{J}_{1}(|m x|)\right]+\vartheta\left(-x^{2}\right) 2 \mathcal{K}_{1}(|m x|)}{\left|\frac{m x}{2}\right|} \\
& +i \epsilon\left(x_{0}\right) \delta\left(\frac{x^{2}}{4} \pi\right) .
\end{aligned}
$$

Feynman propagators are expectation values of time-ordered products with an explicitly spacetime-dependent order factor $\epsilon\left(x_{0}-y_{0}\right)$ :

$$
\frac{\{\boldsymbol{\Phi}(y) \boldsymbol{\Phi}(x)\}-\epsilon\left(x_{0}-y_{0}\right)[\boldsymbol{\Phi}(y), \boldsymbol{\Phi}(x)]}{2}=\vartheta\left(x_{0}-y_{0}\right) \boldsymbol{\Phi}(x) \boldsymbol{\Phi}(y)+\vartheta\left(y_{0}-x_{0}\right) \boldsymbol{\Phi}(y) \boldsymbol{\Phi}(x) .
$$

The creation-annihilation operator assignment is compatible with the time order: annihilation is possible only after creation.

The particle induced off-shell energy-momentum distributions of the Feynman propagators for the interactions are no translation coefficients, and not convolutable; their undefined products cause the divergencies in the perturbation expansion of an interaction with canonically quantized particle related fields. The translations incompatible interactions are representation distributions, i.e., linear mappings of representation functions. They can be added, however not multiplied with each other: The pointwise product of two interactions, e.g., of two Yukawa potentials $\frac{e^{-\left|B_{1}\right| r}}{r} \cdot \frac{e^{-\left|B_{2}\right| r}}{r}=\frac{e^{-\left|B_{+}\right| r}}{r^{2}}$, or of their relativistic 
embeddings, e.g., $\frac{1}{r} \hookrightarrow \delta\left(x^{2}\right)$ and $\delta\left(x^{2}\right) \cdot \delta\left(x^{2}\right)$, does not make sense as an interaction. The convolution of two scalar Feynman propagators with general real power denominators, $v_{1,2} \in \mathbb{R}$ (more ahead):

$$
\begin{aligned}
\Pi\left(m_{1}^{2}, v_{1}, m_{2}^{2}, v_{2} ; q^{2}\right) & =\int d^{4} q_{1} d^{4} q_{2} \frac{\Gamma\left(1+v_{1}\right)}{\left(-q_{1}^{2}-i o+m_{1}^{2}\right)^{1+v_{1}}} \frac{\delta\left(q_{1}+q_{2}-q\right)}{i \pi^{2}} \frac{\Gamma\left(1+v_{2}\right)}{\left(-q_{2}^{2}-i o+m_{2}^{2}\right)^{1+v_{2}}} \\
& =\int_{0}^{1} d z \frac{z^{v_{1}}(1-z)^{v_{2}} \Gamma\left(v_{1}+v_{2}\right)}{\left[-z(1-z) q^{2}-i o+z m_{1}^{2}+(1-z) m_{2}^{2}\right]^{v_{1}+v_{2}}}
\end{aligned}
$$

is not defined for non-positive integers $v_{1}+v_{2}=0,-1, \ldots$. The convolution of the propagators of a positively and negatively charged pion from the pointwise product of distributions $\left\langle\Phi \Phi^{\star}\right\rangle(x) \cdot\left\langle\Phi \Phi^{\star}\right\rangle(x)$ occurs in the renormalization of the coupling constant of the electromagnetic field by the vacuum polarization $\Pi\left(m^{2}, v_{1}, m^{2}, v_{2} ; 0\right)=$ $\frac{\Gamma\left(1+v_{1}\right) \Gamma\left(1+v_{2}\right)}{\Gamma\left(2+v_{1}+v_{2}\right)} \frac{\Gamma\left(v_{1}+v_{2}\right)}{\left(m^{2}-i o\right)^{v_{1}+v_{2}}}$, undefined ("divergent") for particle propagators with $v_{1,2}=0$.

The convolution of the on-shell contributions (relativistic phase space integral) is well defined:

$$
\begin{array}{r}
\int d^{4} q_{1} d^{4} q_{2} \vartheta\left( \pm q_{10}\right) \delta\left(q_{1}^{2}-m_{1}^{2}\right) \frac{\delta\left(q_{1}+q_{2}-q\right)}{4 \pi} \vartheta\left( \pm q_{20}\right) \delta\left(q_{2}^{2}-m_{2}^{2}\right) \\
=\frac{\sqrt{\Delta\left(q^{2}\right)}}{4 q^{2}} \vartheta\left( \pm q_{0}\right) \vartheta\left(q^{2}-m_{+}^{2}\right) .
\end{array}
$$

It contains the threshold factor (discriminant) $\Delta$, symmetric in the three translation invariants $\left\{q^{2}, m_{1}^{2}, m_{2}^{2}\right\}$ :

$$
\begin{aligned}
-z(1-z) q^{2}+z m_{1}^{2}+ & (1-z) m_{2}^{2}=0 \Rightarrow z_{1,2}=\frac{q^{2}-m_{+} m_{-} \pm \sqrt{\Delta\left(q^{2}\right)}}{2 q^{2}} \\
\Delta\left(q^{2}\right) & =\left(q^{2}-m_{+}^{2}\right)\left(q^{2}-m_{-}^{2}\right) \\
& =\left(q^{2}\right)^{2}+m_{1}^{4}+m_{2}^{4}-2\left(q^{2} m_{1}^{2}+m_{1}^{2} m_{2}^{2}+m_{2}^{2} q^{2}\right) \\
\text { with } m_{ \pm} & =\left|m_{1}\right| \pm\left|m_{2}\right| .
\end{aligned}
$$

\section{Spherical and Hyperbolic Invariants for Indefinite Orthogonal Groups}

Orthogonal groups for real even- and odd-dimensional spaces constitute different Cartan series. They come in pairs with respect to the rank, i.e., the number of independent invariants: The Lie algebras $\log \mathbf{S} \mathbf{O}_{0}(t, s)$, both with even $t+s=2 R=2,4, \ldots$ (Cartan series $D_{R}$ ) and with odd $t+s=1+2 R=3,5, \ldots$ (Cartan series $B_{R}$ ), have rank $R=1,2, \ldots$. Two additional real dimensions $t+s+2$ are necessary to raise the rank by one $R+1$. This becomes relevant for the Lorentz groups where $\mathbf{S O}_{0}(1,2 R-1)$ with odddimensional position has a unique Cartan subgroup type - a noncompact Cartan cylinder $S \boldsymbol{O}_{0}(1,1) \times \boldsymbol{S O}(2)^{R-1}$, e.g., the proper Lorentz group $\mathbf{S O}_{0}(1,3)$ with self-dual dilations and axial rotations $\left(\begin{array}{cc|cc}\cosh \psi & \sinh \psi & 0 & 0 \\ \sinh \psi & \cosh \psi & 0 & 0 \\ \hline 0 & 0 & \cos \theta & -\sin \theta \\ 0 & 0 & \sin \theta & \cos \theta\end{array}\right) \in \mathbf{S O}_{0}(1,1) \times \mathbf{S O}(2)$, whereas $\mathbf{S O}_{0}(1,2 R)$ with even-dimensional position has two types of Cartan subgroups, either a compact Cartan torus $\boldsymbol{S} \boldsymbol{O}(2)^{R}$ or a Cartan cyclinder $\mathbf{S O}_{0}(1,1) \times \mathbf{S O}(2)^{R-1}$, e.g., for $\mathbf{S O}_{0}(1,2)$ with either $\left(\begin{array}{c|cc}1 & 0 & 0 \\ \hline 0 & \cos \theta & -\sin \theta \\ 0 & \sin \theta & \cos \theta\end{array}\right) \in \mathbf{S O}(2)$ or $\left(\begin{array}{cc|c}\cosh \psi & \sinh \psi & 0 \\ \sinh \psi & \cosh \psi & 0 \\ \hline 0 & 0 & 1\end{array}\right) \in \mathbf{S O}_{0}(1,1)$. The difference between odd- and even-dimensional spacetimes shows up also in the implementation of either only 
imaginary (compact) or only real (noncompact) infinitesimal operations, or of all complex operations, $\mathbb{R}+i \mathbb{R}=\mathbb{C}$, as seen for the "smallest" nonabelian Lorentz groups with their Cartan subgroups:

$$
\begin{aligned}
& \mathbf{S L}(2, \mathbb{R}) \sim \mathbf{S O}_{0}(1,2) \text { with }\left\{\begin{aligned}
\text { either } \mathbf{S O}(2) & \cong \exp i \mathbb{R} \ni e^{i \sigma_{3} \theta}, \\
\text { or } \mathbf{S O}_{0}(1,1) & \cong \exp \mathbb{R} \ni e^{\sigma_{3} \psi},
\end{aligned}\right. \\
& \mathbf{S L}(2, \mathbb{C}) \sim \mathbf{S O}_{0}(1,3) \text { withSO}(2) \times \mathbf{S O}_{0}(1,1) \cong \mathbf{U}(1) \times \mathbf{D}(1) \cong \exp \mathbb{C} .
\end{aligned}
$$

The indefinite orthogonal group for the metric $q^{2}=\vec{q}_{t}^{2}-\vec{q}_{s}^{2}$ has an Iwasawa factorization with two compact factor subgroups for opposite definiteness:

$$
1 \leq t \leq s: \mathbf{O}(t, s)=[\mathbf{O}(t) \times \mathbf{O}(s)] \circ \mathbf{S O}_{0}(1,1)^{t} \circ \exp \mathbb{R}^{t(s-1)} .
$$

Maximally symmetric spacetimes with $(t, s)$-metric are characterized by a constant curvature and one invariant (unit). In the not maximally symmetric cases with an invariant (unit) for both metrical sectors, one uses the $\varnothing(t, s)$-invariantly embedded $\mathbf{O}(t)$-distribution $\frac{d^{t} q \Gamma\left(1+R_{t}\right)}{\left(-\vec{q}_{t}^{2}+m_{t}^{2}\right)^{1+R_{t}}} \hookrightarrow \frac{d^{n} q \Gamma\left(1+R_{t}\right)}{\left(-q^{2}+m_{t}^{2}\right)^{1+R_{t}}}$ (spherical invariant $\vec{q}_{t}^{2}=m_{t}^{2}>0$ for negative $\mathbf{O}(t)$ definiteness) and $\mathbf{O}(s)$-distribution $\frac{d^{s} q \Gamma\left(1+R_{s}\right)}{\left(\vec{q}_{s}^{2}+m_{s}^{2}\right)^{1+R_{s}}} \hookrightarrow \frac{d^{n} q \Gamma\left(1+R_{s}\right)}{\left(-q^{2}+m_{s}^{2}\right)^{1+R_{s}}}$ (hyperbolic invariant $\vec{q}_{s}^{2}=-m_{s}^{2}<0$ for positive $\mathbf{O}(s)$-definiteness) where the point invariants for the subgroups are distributed on lines: $-\vec{q}_{t}^{2}+m_{t}^{2} \hookrightarrow-\vec{q}_{t}^{2}+\left(m_{t}^{2}+\vec{q}_{s}^{2}\right)$ and $\vec{q}_{s}^{2}+m_{s}^{2} \hookrightarrow \vec{q}_{s}^{2}+\left(m_{s}^{2}-\vec{q}_{t}^{2}\right)$. The group product related convolution product of the two subgroup spacetime distributions leads to the pointwise product of the two "energy-momentum" functions in the scalar representation coefficients:

$$
\begin{array}{rl}
\mathbf{O}(t) \times \mathbf{O}(s) & \hookrightarrow \mathbf{O}(t, s), \\
\int d^{n} q \frac{\Gamma\left(1+R_{t}\right)}{\left(-q^{2}+m_{t}^{2}\right)^{1+R_{t}}} e^{i q x} & * \int d^{n} q \frac{\Gamma\left(1+R_{s}\right)}{\left(-q^{2}+m_{s}^{2}\right)^{1+R_{s}}} e^{i q x} \\
& \sim \int d^{n} q \frac{\Gamma\left(1+R_{t}\right) \Gamma\left(1+R_{s}\right)}{\left(-q^{2}+m_{t}^{2}\right)^{1+R_{t}}\left(-q^{2}+m_{s}^{2}\right)^{1+R_{s}}} e^{i q x} .
\end{array}
$$

$\mathbf{O}(t, s)$-scalar integrals, $1 \leq t \leq s$, with two invariants are obtained by 2 -sphere spreads from $\mathbf{O}(1,1)$-, $\mathbf{O}(1,2)$ - and $\mathbf{O}(2,2)$-scalar integrals with odd-odd, odd-even and even-even subdimensions and distributed subgroups $\mathbf{O}(1) \times \mathbf{O}(1), \mathbf{O}(1) \times \mathbf{O}(2)$ and $\mathbf{O}(2) \times \mathbf{O}(2)$, respectively.

For nontrivial signature with odd-odd subdimensions adding up to even dimensions,

$$
\begin{gathered}
\mathbf{O}(t, s)=\mathbf{O}\left(1+2 R_{t}, 1+2 R_{s}\right) \quad \text { with } n=t+s=2\left(1+R_{t}+R_{s}\right), \\
\text { for } R_{t}, R_{s}=0,1,2, \ldots,
\end{gathered}
$$

one has the characteristic scalar representation coefficients for cyclic (ground) states:

$$
\begin{aligned}
& \int \frac{d^{2\left(1+R_{t}+R_{s}\right)} q}{\pi^{1+R_{t}+R_{s}}} \frac{\Gamma\left(1+R_{t}\right)}{\left(-q^{2}-i o+m_{t}^{2}\right)^{1+R_{t}}} \frac{\Gamma\left(1+R_{s}\right)}{\left(-q^{2}-i o+m_{s}^{2}\right)^{1+R_{s}}} e^{i q x} \\
& =\int_{0}^{1} d z z^{R_{s}}(1-z)^{R_{t}} \int \frac{d^{2\left(1+R_{t}+R_{s}\right)} q}{\pi^{1+R_{t}+R_{s}}} \frac{\Gamma\left(2+R_{t}+R_{s}\right)}{\left(-q^{2}-i o+m_{z}^{2}\right)^{2+R_{t}+R_{s}}} e^{i q x} \\
& =\int_{m_{s}^{2}}^{m_{t}^{2}} \frac{d m^{2}}{m_{t}^{2}-m_{s}^{2}} \frac{\left(m^{2}-m_{s}^{2}\right)^{R_{t}}\left(m_{t}^{2}-m^{2}\right)^{R_{s}}}{\left(m_{t}^{2}-m_{s}^{2}\right)^{R_{t}+R_{s}}} \int \frac{d^{2 R} q}{\pi^{R}} \frac{\Gamma(1+R)}{\left(-q^{2}-i o+m^{2}\right)^{1+R}} e^{i q x}, \\
& \quad \text { with } R=1+R_{t}+R_{s}, m_{z}^{2}=(1-z) m_{t}^{2}+z m_{s}^{2}=m^{2} .
\end{aligned}
$$


The denominators are joined with the generalized Beta function relation, adjusting pole orders and Gamma function arguments:

$$
\begin{aligned}
\frac{\Gamma\left(v_{1}\right)}{D_{1}^{\nu_{1}}} \frac{\Gamma\left(v_{2}\right)}{D_{2}^{\nu_{2}}} & =\int_{0}^{1} d z(1-z)^{v_{1}-1} z^{\nu_{2}-1} \frac{\Gamma\left(v_{1}+v_{2}\right)}{\left[(1-z) D_{1}+z D_{2}\right]^{\nu_{1}+v_{2}}} \\
& =\int_{D_{2}}^{D_{1}} d D \frac{\left(D-D_{2}\right)^{\nu_{1}-1}\left(D_{1}-D\right)^{\nu_{2}-1}}{\left(D_{1}-D_{2}\right)^{\nu_{1}+v_{2}-1}} \frac{\Gamma\left(v_{1}+v_{2}\right)}{D^{v_{1}+v_{2}}}
\end{aligned}
$$

The Lie algebra of $\mathbf{O}\left(1+2 R_{t}, 1+2 R_{S}\right)$ has rank $R=1+R_{t}+R_{S}$ - one genuine noncompact $\mathbf{O}(1,1)$-related real rank in addition to the sum of the imaginary ranks $R_{t, s}$ for its compact subgroups $\mathbf{O}\left(1+2 R_{t, s}\right)$. The integral $\int_{m_{s}^{2}}^{m_{t}^{2}} d m^{2}$ sums up a line singularity for the $\mathbf{O}(t, s)$-invariant $q^{2}=m^{2}$ with the invariant for $x=0$ in the minimal case showing the characteristic logarithmic connection of spherical and hyperbolic invariant:

$$
\begin{aligned}
\mathbf{O}(1) \times \mathbf{O}(1) \hookrightarrow \mathbf{O}(1,1): \int_{m_{s}^{2}}^{m_{t}^{2}} \frac{d m^{2}}{m_{t}^{2}-m_{s}^{2}} \int \frac{d^{2} q}{i \pi} \frac{1}{\left(-q^{2}-i o+m^{2}\right)^{2}} & =\frac{1}{m_{t}^{2}-m_{s}^{2}} \int_{m_{s}^{2}}^{m_{t}^{2}} \frac{d m^{2}}{m^{2}} \\
& =\frac{1}{m_{t}^{2}-m_{s}^{2}} \log \frac{m_{t}^{2}}{m_{s}^{2}} .
\end{aligned}
$$

For odd-even $\mathbf{O}\left(1+2 R_{t}, 2 R_{s}\right)$, starting with $\mathbf{O}(1,2)$, and even-even subdimensions $\mathbf{O}\left(2 R_{t}, 2 R_{s}\right)$, starting with $\mathbf{O}(2,2)$, the subgroups-related rank $R_{t}+R_{s}$ stays constant.

Important Lorentz group examples are even-dimensional causal spacetimes embedding odd-dimensional positions, with $q^{2}=q_{0}^{2}-\vec{q}^{2}$ :

$$
\begin{aligned}
& \mathbf{O}(1): \int \frac{d q_{0}}{i \pi} \frac{1}{-q_{0}^{2}-i o+m_{t}^{2}} e^{i q_{0} x_{0}}=\frac{e^{-i\left|m_{t} x_{0}\right|}}{\left|m_{t}\right|}, R_{t}=0, \\
& \mathbf{O}\left(1+2 R_{s}\right): \int \frac{d^{1+2 R_{s}} q}{\pi^{1+R_{s}}} \frac{\Gamma\left(1+R_{s}\right)}{\left(\vec{q}^{2}+m_{s}^{2}\right)^{1+R_{s}}} e^{i \vec{q} \vec{x}}=\frac{e^{-\left|m_{s}\right| r}}{\left|m_{s}\right|}, R_{s}=R-1=0,1,2, \ldots, \\
& \mathbf{O}(1,2 R-1): \int \frac{d^{2 R} q}{i \pi^{R}} \frac{\Gamma(R)}{\left(-q^{2}-i o+m_{t}^{2}\right)\left(-q^{2}-i o+m_{s}^{2}\right)^{R}} e^{i q x} \\
& =\int_{0}^{1} d z z^{R-1} \int \frac{d^{2 R} q}{i \pi^{R}} \frac{\Gamma(1+R)}{\left(-q^{2}-i o+m_{z}^{2}\right)^{1+R}} e^{i q x} \\
& =\int_{m_{s}^{2}}^{m_{t}^{2}} \frac{d m^{2}}{m_{t}^{2}-m_{s}^{2}}\left(\frac{m_{t}^{2}-m^{2}}{m_{t}^{2}-m_{s}^{2}}\right)^{R-1} \int_{m^{2}}^{\infty} \frac{d \kappa^{2}}{2 \sqrt{\left(\kappa^{2}-m^{2}\right)^{3}}} \frac{e^{-\sqrt{-\kappa^{2} x^{2}+i o}}}{|\kappa|} .
\end{aligned}
$$

The normalization of these representations will be discussed ahead.

\section{Electroweak Spacetime}

The standard model of the elementary interactions in Minkowski spacetime is a theory of compatibly represented external and internal operations. It embeds the historically first formulated quantum field theory for the electromagnetic interaction of an electron Dirac field (quantum electrodynamics) into the electroweak and strong gauge interactions of quark and lepton Weyl fields. The fields involved are acted on by irreducible representations $(L \mid R)$ of the Lorentz group $\mathbf{S L}(2, \mathbb{C})$ and irreducible representations of the hypercharge group 
$\mathbf{U}(1)$ (rational hypercharge number $y$ ), of the isospin group $\mathbf{S U}(2)$ (integer or halfinteger isospin $T$ ), and the color group $\mathbf{S U}(3)$ as given in the following Table 1:

The complex functions of electroweak spacetime are the coefficients of unitary relativity:

$$
\begin{aligned}
\mathbf{G L}(2, \mathbb{C}) & =\mathbf{U}(2) \circ \mathbf{D}(1) \circ \mathbf{S O}_{0}(1,1) \circ \exp \mathbb{R}^{2}, \\
\mathbf{G L}(2, \mathbb{C}) / \mathbf{U}(2) & =\mathbb{D}(2) \ni d \longmapsto \varphi(d) \in \mathbb{C} .
\end{aligned}
$$

The hyperisospin $\mathbf{U}(2)$-induced representations of the hyper-Lorentz group $\mathbf{G L}(2, \mathbb{C})$ are subrepresentations $\mathbf{G L}(2, \mathbb{C}) \times \mathbf{U}(2) \subset \mathbf{G L}(2, \mathbb{C}) \times \mathbf{G L}(2, \mathbb{C})$ of the two-sided regular representation. They act on $\mathbf{U}(2)$-intertwiners, i.e., on mappings that connect spacetime points, parametrizing unitary classes, with hyperisospin orbits in a Hilbert space with $\mathbf{U}(2)$-representation. With respect to the homogeneous operations, fields of electroweak spacetime are transmutators $\varphi$ for the transition $\mathbf{U}(2) \subset \mathbf{G L}(2, \mathbb{C})$ from the internal hyperisospin group to the external hyper-Lorentz group. They associate each hyperisospin orbit in the hyper-Lorentz group, parametrized by four real parameters $g \mathbf{U}(2) \cong d \cong\left(d_{\mu}\right)_{\mu=0}^{3} \in$ $\mathbb{D}(2)$, with linear mappings $\{V \longrightarrow W\}$ from a vector space $V$ with $\mathbf{U}(2)$-action to a vector space $W$ with $\mathbf{G L}(2, \mathbb{C})$-action:

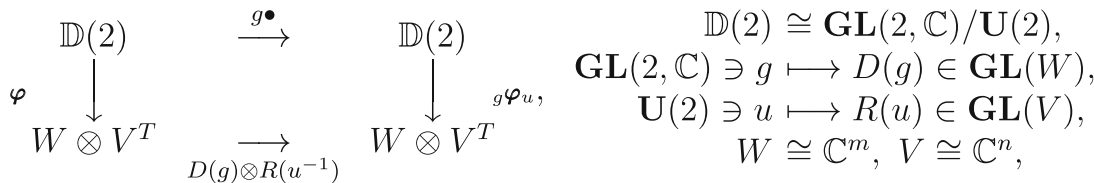

$$
\begin{aligned}
& d \longmapsto \varphi(d) \text { with }{ }_{g} \varphi_{u}(d)=D(g) \circ \varphi\left(g^{-1} \bullet d\right) \circ R\left(u^{-1}\right)
\end{aligned}
$$

In the bi-regular representation the subgroup $\mathbf{S U}(2)$ acts twice, i.e., independently for the pair spin-isospin $\mathbf{S U}(2) \times \mathbf{S U}(2)$ with isomorphic groups.

The action group $\mathbf{S L}(2, \mathbb{C}) \times \mathbf{U}(2)$ with external and internal transformations as subgroup of the both-sided regular $\mathbf{G L}(2, \mathbb{C})$-action is realized as homogeneous group in the electroweak standard model: The representations act faithfully for $\mathbf{S L}(2, \mathbb{C}) \times \mathbf{U}(2)$ on the

\begin{tabular}{|c|c|c|c|c|c|c|}
\hline Field & Symbol & $\begin{array}{l}\mathbf{S L}(2, \mathbb{C}) \\
(L \mid R)\end{array}$ & $\begin{array}{l}\mathbf{U}(1) \\
y\end{array}$ & $\begin{array}{l}\mathbf{S U}(2) \\
(T)\end{array}$ & $\begin{array}{l}\mathbf{S U}(3) \\
{\left[C_{1}, C_{2}\right]}\end{array}$ & $\begin{array}{l}\mathbf{U}(1)_{+} \\
q=y+T_{3}\end{array}$ \\
\hline Left lepton & 1 & $\left(\frac{1}{2} \mid 0\right)$ & $-\frac{1}{2}$ & $\left(\frac{1}{2}\right)$ & {$[0,0]$} & $0,-1$ \\
\hline "Mute" neutrino & $v$ & $\left(0 \mid \frac{1}{2}\right)$ & 0 & $(0)$ & {$[0,0]$} & 0 \\
\hline Right lepton & $\mathbf{e}$ & $\left(0 \mid \frac{1}{2}\right)$ & -1 & (0) & {$[0,0]$} & -1 \\
\hline Left quark & $\mathbf{q}$ & $\left(\frac{1}{2} \mid 0\right)$ & $\frac{1}{6}$ & $\left(\frac{1}{2}\right)$ & {$\left[\frac{1}{2}, 0\right]$} & $\frac{2}{3},-\frac{1}{3}$ \\
\hline Right up quark & $\mathbf{u}$ & $\left(0 \mid \frac{1}{2}\right)$ & $\frac{2}{3}$ & (0) & {$\left[\frac{1}{2}, 0\right]$} & $\frac{2}{3}$ \\
\hline Right down quark & d & $\left(0 \mid \frac{1}{2}\right)$ & $-\frac{1}{3}$ & (0) & {$\left[\frac{1}{2}, 0\right]$} & $-\frac{1}{3}$ \\
\hline Hypercharge gauge & $\mathbf{A}_{0}$ & $\left(\frac{1}{2} \mid \frac{1}{2}\right)$ & 0 & (0) & {$[0,0]$} & 0 \\
\hline Isospin gauge & $\overrightarrow{\mathbf{A}}$ & $\left(\frac{1}{2} \mid \frac{1}{2}\right)$ & 0 & (1) & {$[0,0]$} & $0, \pm 1$ \\
\hline Color gauge & C & $\left(\frac{1}{2} \mid \frac{1}{2}\right)$ & 0 & (0) & {$\left[\frac{1}{2}, \frac{1}{2}\right]$} & 0 \\
\hline Higgs & $\Phi$ & $(0 \mid 0)$ & $\frac{1}{2}$ & $\left(\frac{1}{2}\right)$ & {$[0,0]$} & 1,0 \\
\hline
\end{tabular}

Table 1 The fields of the minimal standard model with representation space dimensions $d_{\mathrm{SL}(2, \mathbb{C})}=(1+$ $2 L)(1+2 R), d_{\mathbf{U}(1)}=1, d_{\mathbf{S U}(2)}=1+2 T, d_{\mathbf{S U}(3)}=\left(1+2 C_{1}\right)\left(1+2 C_{2}\right)\left(1+C_{1}+C_{2}\right)$ 
left-handed spinor-isospinor lepton field $\mathbb{R}^{(1,3)} \ni x \longmapsto \mathbf{l}_{\alpha}^{A}(x) \in \mathbb{C}^{2} \otimes \mathbb{C}^{2}$ with Feynman propagator $\left\langle\mathbf{I I}^{\star}\right\rangle(x) \sim \int \frac{d^{4} q}{i \pi^{2}} \frac{\sigma^{j} q_{j} \times \mathbf{1}_{2}}{-q^{2}-i o+m^{2}} e^{i q x}$, for its adjoint group $\mathbf{S O}_{0}(1,3) \times \mathbf{S O}(3)$ on the isotriplet gauge vector field $x \longmapsto \mathbf{A}_{a}^{j}(x) \in \mathbb{C}^{4} \otimes \mathbb{C}^{3}$, for $\mathbf{S L}(2, \mathbb{C}) \times \mathbf{U}(1)$ on the right-handed isosinglet lepton field $x \longmapsto \mathbf{r}^{\dot{A}}(x) \in \mathbb{C}^{2} \otimes \mathbb{C}$, and for its adoint group $\mathbf{S O}_{0}(1,3) \times\{1\}$ on the hypercharge gauge vector field $x \longmapsto \mathbf{A}_{0}^{j}(x) \in \mathbb{C}^{4} \otimes \mathbb{C}$. The $\mathbf{S L}(2, \mathbb{C})$-Lorentz group representations are induced by the $\mathbf{S U}(2)$-isospin representations: With the notable exception of the Lorentz scalar isodublet Higgs field, all isospin SU(2)representations of the standard model fields are isomorphic to subrepresentations of their Lorentz group $\mathbf{S L}(2, \mathbb{C})$-representations with $V \cong \mathbb{C}^{n} \subseteq \mathbb{C}^{m} \cong W$ which can be related to Frobenius' reciprocity for induced representations.

\subsection{Dilation and Translation Parametrizations of Causal Spacetimes}

In the following, electroweak spacetime $\mathbb{D}(2)=\mathcal{D}^{4} \cong \mathbf{G L}(2, \mathbb{C}) / \mathbf{U}(2)$ will be considered as the $(R=2)$-member of the series with the even-dimensional causal spacetimes for the classes of the odd-dimensional orthogonal groups:

$$
\begin{aligned}
R=1,2, \ldots: \mathcal{D}^{2 R} & \cong \mathbf{D}(1) \times \mathcal{Y}^{2 R-1} \cong \exp \mathbb{R}^{(1,2 R-1)}, \\
\mathbf{D}(1) & \cong \exp \mathbb{R}, \\
\mathcal{Y}^{2 R-1} & \cong \exp \mathbb{R}^{2 R-1} \cong \mathbf{S O}_{0}(1,2 R-1) / \mathbf{S O}(2 R-1) .
\end{aligned}
$$

Since the maximal noncompact abelian motion group $\mathcal{D}^{2}=\mathbf{D}(1) \times \mathbf{S O}_{0}(1,1)$, called Cartan spacetime, is two-dimensional, $\mathcal{D}^{2 R}$ has real rank two.

Electroweak spacetime is also the $(n=2)$-member in the series of the causal manifolds for the unitary group classes:

$$
n=1,2, \ldots: \mathbb{D}(n)=\mathbf{G L}(n, \mathbb{C}) / \mathbf{U}(n)=\mathbf{D}(1) \times \mathbf{S L}(n, \mathbb{C}) / \mathbf{S U}(n) .
$$

This series starts with the dilation (causal) group $\mathbf{D}(1) . \mathbb{D}(n) \cong \exp \mathbb{R}(n)$ has real dimension $n^{2}$ and real rank $n \cdot \mathcal{D}^{2 R}$ and $\mathbb{D}(n)$ meet only for four dimensions, i.e., $\mathcal{D}^{4} \cong \mathbb{D}(2) \cong$ $\mathbf{D}(1) \times \mathcal{Y}^{3}$.

Two analogue series contain the compact partners with imaginary ranks and rational invariants:

$$
\begin{aligned}
\mathcal{D}^{2 R} \leftrightarrow \mathcal{U}^{2 R} \cong \mathbf{U}(1) \times \mathbf{S O}(2 R) / \mathbf{S O}(2 R-1) \cong \mathbf{U}(1) \times \Omega^{2 R-1}, \\
\mathbb{D}(n) \leftrightarrow \mathbb{U}(n) \cong[\mathbf{U}(n) \times \mathbf{U}(n)] / \mathbf{U}(n) .
\end{aligned}
$$

with $\mathcal{U}^{4} \cong \mathbb{U}(2) \cong \mathbf{U}(1) \times \Omega^{3}$.

The additive reals (translations) $\mathbb{R}$ and its multiplicative exponentials (dilations) $\mathbf{D}(1)=$ $\exp \mathbb{R}$ with Lie algebra $\log \mathbf{D}(1)=\mathbb{R}$ are embedded in higher dimensional causal spacetimes $\mathcal{D}^{2 R}$ with corresponding Minkowski spacetime $\mathbb{R}^{(1,2 R-1)}$ as tangent spaces:

$$
\begin{aligned}
& \mathbb{R}^{(1,2 R-1)} \cong \log \left[\mathbf{D}(1) \times \mathbf{S O}_{0}(1,2 R-1)\right] / \log \mathbf{S O}(2 R-1) \\
& \cong\left[\mathbf{S O}_{0}(1,2 R-1) \overrightarrow{\times} \mathbb{R}^{2 R}\right] / \mathbf{S O}_{0}(1,2 R-1), \\
& \mathcal{D}^{2 R}=\left\{d=\left(\begin{array}{l}
d_{0} \\
\vec{d}
\end{array}\right)=e^{\psi_{0}}\left(\begin{array}{l}
\cosh |\vec{\psi}| \\
\sinh |\vec{\psi}| \vec{\omega}_{2 R-2}
\end{array}\right)\left|\vec{\omega}_{2 R-2}=\frac{\vec{\psi}}{|\vec{\psi}|} \in \Omega^{2 R-2}, \psi_{0} \in \mathbb{R},\right| \vec{\psi} \mid \in \mathbb{R}_{+}\right\}, \\
& \text {with } e^{\psi_{0}}=|d|=\sqrt{d_{0}^{2}-\vec{d}^{2}}, e^{|\vec{\psi}|}=\sqrt{\frac{d_{0}+|\vec{d}|}{d_{0}-|\vec{d}|}} \text {. }
\end{aligned}
$$


An example is the Weyl representation of electroweak spacetime by exponentials of the spacetime translations, given by hermitian $(2 \times 2)$-matrices:

$$
\begin{aligned}
& \mathbf{G L}(2, \mathbb{C}) \times \mathbb{D}(2) \longrightarrow \mathbb{D}(2), \mathbf{G L}(2, \mathbb{C}) \times \mathbb{R}(2) \rightarrow \mathbb{R}(2), \\
& \mathbf{G L}(2, \mathbb{C})=\exp \mathbf{A L}(2, \mathbb{C}) \supset \mathbb{D}(2)=\exp \mathbb{R}(2), \\
& \mathbb{R}^{(1,3)}=\mathbb{R}(2) \ni \psi=\left(\begin{array}{ll}
\psi_{0}+\psi_{3} & \psi_{1}-i \psi_{2} \\
\psi_{1}+i \psi_{2} & \psi_{0}-\psi_{3}
\end{array}\right)=u \circ\left(\begin{array}{cc}
\psi_{0}+|\vec{\psi}| & 0 \\
0 & \psi_{0}-|\vec{\psi}|
\end{array}\right) \circ u^{\star} \\
& \quad \mathcal{D}^{4}=\mathbb{D}(2) \ni e^{\psi}=e^{\psi_{0}}\left(\cosh |\vec{\psi}| \mathbf{1}_{2}+\frac{\sinh |\vec{\psi}|}{|\vec{\psi}|} \vec{\sigma}\right) \\
& \quad=d=\left(\begin{array}{ll}
d_{0}+d_{3} & d_{1}-i d_{2} \\
d_{1}+i d_{2} & d_{0}-d_{3}
\end{array}\right)=u \circ\left(\begin{array}{cc}
d_{0}+|\vec{d}| & 0 \\
0 & d_{0}-|\vec{d}|
\end{array}\right) \circ u^{\star}, d_{0} \pm|\vec{d}|>0
\end{aligned}
$$

with the Cartan subgroup and the rotations:

$$
\begin{aligned}
& \mathbf{D}(1) \times \mathbf{S O}_{0}(1,1) \ni\left(\begin{array}{ll}
d_{0}+|\vec{d}| & 0 \\
0 & d_{0}-|\vec{d}|
\end{array}\right)=\left(\begin{array}{ll}
e^{\psi_{0}+|\vec{\psi}|} & 0 \\
0 & e^{\psi_{0}-|\vec{\psi}|}
\end{array}\right), \\
& \Omega^{2} \ni \vec{\omega}_{2}=\frac{\vec{d}}{|\vec{d}|}=\frac{\vec{\psi}}{|\vec{\psi}|} \longmapsto u=\left(\begin{array}{ll}
\cos \frac{\theta}{2} & -e^{-i \phi} \sin \frac{\theta}{2} \\
e^{i \phi} \sin \frac{\theta}{2} & \cos \frac{\theta}{2}
\end{array}\right) \\
& =\frac{1}{\sqrt{2|\vec{\psi}|\left(\psi_{3}+|\vec{\psi}|\right)}}\left(\begin{array}{cc}
\psi_{3}+|\vec{\psi}| & -\psi_{1}+i \psi_{2} \\
\psi_{1}+i \psi_{2} & \psi_{3}+|\vec{\psi}|
\end{array}\right) \\
& \in \mathbf{S U}(2) \text {. }
\end{aligned}
$$

Ahead, the dimensionless parameters obtain units via the invariants of the group representations.

In contrast to time $\mathbf{D}(1)$, electroweak spacetime $\mathbb{D}(2)=\exp \mathbb{R}(2)$ is no group. The noncompact dilations $\mathbf{D}(1)$ constitute, in a relativistic framework, the group for the Lorentz invariant "eigentime" $e^{\psi_{0}} \in \mathbf{D}(1)$. The noncompact odd-dimensional hyperboloids are the position submanifolds $\left(\begin{array}{l}\cosh |\vec{\psi}| \\ \sinh |\vec{\psi}| \vec{\omega}_{2 R-2}\end{array}\right) \in \mathcal{Y}^{2 R-1}$.

Spacetime $\mathcal{D}^{2 R}$ is an orbit of $\mathbf{D}(1) \times \mathbf{S O}_{0}(1,2 R-1)$. For the future cone, foliated by position hyperboloids $\mathcal{Y}^{2 R-1}$, the action of the causal group $\mathbf{D}(1)$ may be called hyperbolic hopping, from position hyperboloid to position hyperboloid, and the action of the dilative Lorentz subgroup $\mathbf{S O}_{0}(1,1) \subseteq \mathbf{S O}_{0}(1,2 R-1)$ hyperbolic stretching, inside each position hyperboloid,

$$
\begin{aligned}
& \mathbf{D}(1):\left(\begin{array}{l}
d_{0} \\
d_{3}
\end{array}\right)=e^{\psi_{0}}\left(\begin{array}{c}
\cosh \psi_{3} \\
\sinh \psi_{3}
\end{array}\right) \longmapsto e^{\chi_{0}}\left(\begin{array}{l}
d_{0} \\
d_{3}
\end{array}\right)=e^{\chi_{0}+\psi_{0}}\left(\begin{array}{c}
\cosh \psi_{3} \\
\sinh \psi_{3}
\end{array}\right), \\
& \mathbf{S O}_{0}(1,1):\left(\begin{array}{l}
d_{0} \\
d_{3}
\end{array}\right)=e^{\psi_{0}}\left(\begin{array}{c}
\cosh \psi_{3} \\
\sinh \psi_{3}
\end{array}\right) \longmapsto\left(\begin{array}{cc}
\cosh \chi_{3} & \sinh \chi_{3} \\
\sinh \chi_{3} & \cosh \chi_{3}
\end{array}\right)\left(\begin{array}{l}
d_{0} \\
d_{3}
\end{array}\right) \\
& =e^{\psi_{0}}\left(\begin{array}{c}
\cosh \left(\chi_{3}+\psi_{3}\right) \\
\sinh \left(\chi_{3}+\psi_{3}\right)
\end{array}\right) \\
& \Rightarrow\left(\begin{array}{l}
d_{0} \\
d_{3}
\end{array}\right)=e^{\psi_{0} \mathbf{1}_{2}+\psi_{3} \sigma_{1}}\left(\begin{array}{l}
1 \\
0
\end{array}\right) .
\end{aligned}
$$

The maximal compact motion group in the Iwazawa decomposition

$$
\mathbf{S O}_{0}(1,2 R-1)=\mathbf{S O}(2 R-1) \circ \mathbf{S O}_{0}(1,1) \circ \exp \mathbb{R}^{2(R-1)},
$$


nontrivial for $2 R=4,6, \ldots$, rotates each position hyperboloid. Cartan spacetime $\mathcal{D}^{2}$ does not know the concepts spin or polarization.

The tangent translation parametrization $\left(x_{0}, \vec{x}\right)$ is appropriate for a relativistic formulation and an embedding $\mathbf{D}(1) \times \mathcal{Y}^{3} \cong \mathbb{R}_{+}^{4} \hookrightarrow \mathbb{R}^{4}$ in tangent Minkowski spacetime structures with particles and a corresponding harmonic analysis (ahead). The manifold isomorphy of the dilation group with the positive numbers $\mathbf{D}(1) \ni d=e^{\psi_{0}} \cong \vartheta(t) t \in \mathbb{R}_{+}$, is embedded in higher dimensions: A parametrization of causal spacetime is possible by the open future cone of the corresponding Minkowski spacetime with the manifold isomorphy:

$$
\begin{aligned}
\mathcal{D}^{2 R} \ni d & \cong \vartheta\left(x_{0}\right) \vartheta\left(x^{2}\right) x \in \mathbb{R}_{+}^{(1,2 R-1)}=\left\{x \in \mathbb{R}^{(1,2 R-1)} \mid x^{2}>0, x_{0}>0\right\}, \\
d=e^{\psi} & =u \circ\left(\begin{array}{ll}
e^{\psi_{0}+|\vec{\partial} s i|} & 0 \\
0 & e^{\psi_{0}-|\vec{\partial} s i|}
\end{array}\right) \circ u^{\star} \cong x=u \circ\left(\begin{array}{ll}
x_{0}+|\vec{x}| & 0 \\
0 & x_{0}-|\vec{x}|
\end{array}\right) \circ u^{\star} .
\end{aligned}
$$

\subsection{The Causal Cartan Plane}

The causal spacetimes $\mathcal{D}^{2 R}$ have real rank 2 as dimension of a Cartan plane $\mathcal{D}^{2}=\mathbf{D}(1) \times$ $\mathbf{S O}_{0}(1,1)$ with causal operations and Lorentz dilations as maximal noncompact abelian group.

The characteristic functions of the causal Cartan line $\mathbf{D}(1)$ and plane $\mathcal{D}^{2}$ (spacetime without rotation degrees of freedom) can be unitarily analyzed (Fourier-transformed) by an advanced "energy(-momentum)" distribution (measure) with a pole at a trivial invariant $q_{0}=0$ and $q^{2}=0$, respectively,

$$
\begin{aligned}
\text { for } \mathbf{D}(1): & \vartheta\left(x_{0}\right) & =\int \frac{d q_{0}}{2 i \pi} \frac{1}{q_{0}-i o} e^{i q_{0} x_{0}}, \\
\text { for } \mathcal{D}^{2}=\mathbf{D}(1) \times \mathbf{S O}_{0}(1,1): & \vartheta(x) \pi & =\int \frac{d^{2} q}{2 \pi} \frac{1}{-(q-i o)^{2}} e^{i q x}, \\
\text { with } \vartheta( \pm x)=\vartheta\left( \pm x_{0}\right) \vartheta\left(x^{2}\right) & \text { for }(q \mp i o)^{2} & =\left(q_{0} \mp i o\right)^{2}-q_{3}^{2} .
\end{aligned}
$$

The Fourier transform of an "energy-momentum" function, holomorphic in the lower (upper) complex "energy" $q_{0}$ plane, i.e., with $q_{0} \mp i o$, is valued in the future (past) cone, i.e., supported by causal line and plane with $\vartheta\left( \pm x_{0}\right)$.

In a Lorentz group action-compatible translation parametrization, the harmonic analysis of the Cartan plane uses $\mathbf{S O}_{0}(1,1)$-invariants $q^{2}=m^{2}$ as "energy-momentum" singularities:

$$
\begin{aligned}
\mathbf{D}(1): \quad\left\{\begin{aligned}
\int \frac{d q_{0}}{2 i \pi} \frac{1}{q_{0}-i o-m} e^{i q_{0} x_{0}} & =\vartheta\left(x_{0}\right) e^{i m x_{0}}, \\
\int \frac{d q_{0}}{2 \pi} \frac{i q_{0}}{-\left(q_{0}-i o\right)^{2}+m^{2}} e^{i q_{0} x_{0}} & =\vartheta\left(x_{0}\right) \cos m x_{0},
\end{aligned}\right. \\
\mathcal{D}^{2}=\mathbf{D}(1) \times \mathbf{S O}_{0}(1,1): \quad \int \frac{d^{2} q}{2 \pi} \frac{1}{-(q-i o)^{2}+m^{2}} e^{i q x}=\vartheta(x) \pi \mathcal{J}_{0}(|m x|),|x|=\sqrt{\left|x^{2}\right|} .
\end{aligned}
$$

The Bessel functions with half-integer index start from cosine oscillations

$$
\mathbb{R} \ni x \longmapsto \cos m x=\int d q|q| \delta\left(q^{2}-m^{2}\right) e^{i q x},
$$

with one invariant $\mathrm{m}^{2}$. They are used for real odd-dimensional spaces, e.g., for onedimensional time and for three-dimensional position, with energies and momenta as eigenvalues. The Bessel functions with integer index are relevant for real even-dimensional 
spaces, e.g., for four-dimensional spacetime. $\mathcal{J}_{0}$ integrates $\mathbb{R}$-representation with a $\mathbf{D}(1)$ measure supported by a line, e.g., $\int_{|m|}^{\infty} d \kappa$ which starts at the invariant $m^{2}$ :

$$
\begin{aligned}
\mathbb{R} \ni x \longmapsto \pi \mathcal{J}_{0}(m x) & =\int d \psi \sin (|m x| \cosh \psi)=2 \int_{|m|}^{\infty} \frac{d \kappa}{\sqrt{\kappa^{2}-m^{2}}} \sin |\kappa x| \\
& =\int_{0}^{\pi} d \theta \cos (|m x| \cos \theta)=2 \int_{0}^{|m|} \frac{d \kappa}{\sqrt{m^{2}-\kappa^{2}}} \cos |\kappa x| .
\end{aligned}
$$

The amplitudes of the $\mathcal{J}_{0}$-oscillations are exponentially damped. $\mathcal{J}_{0}$ embeds both spherical and hyperbolic representation coefficients, disentanglable for causal Cartan $(1,1)$ spacetime: the time projection yields an oscillating coefficient of the causal operations $\mathbf{D}(1)$ whereas the position projection gives an exponentially decreasing coefficient of the Lorentz dilations $\mathbf{S O}_{0}(1,1)$ :

$$
\begin{aligned}
& \mathcal{D}^{2} \longrightarrow \mathbf{D}(1), \quad \text { with } \vartheta\left(x_{0}\right) x_{0} \longmapsto \int d x_{3} \vartheta(x) \mathcal{J}_{0}(|m x|)=\vartheta\left(x_{0}\right) \frac{\sin m x_{0}}{m}, \\
& \mathcal{D}^{2} \longrightarrow \mathbf{S O}_{0}(1,1), \quad \text { with } x_{3} \longmapsto \int d x_{0} \vartheta(x) \mathcal{J}_{0}(|m x|)=\frac{e^{-\left|m x_{3}\right|}}{|m|} .
\end{aligned}
$$

The spherical and hyperbolic invariants for the $\mathbf{D}(1)$-oscillations and the $\mathbf{S O}_{0}(1,1)$-falloff must not coincide. Two continuous invariants (units) for the rank 2 causal plane are implementable, in a residual representation, by two poles at $( \pm|m|)$ and $( \pm i|M|)$ in the complex energy and momentum planes with the pointwise product of two functions for hyperbolic non-relativistic space-time:

$$
\begin{aligned}
\mathbf{D}(1) \times \mathbf{S O}_{0}(1,1) \ni\left(\vartheta\left(x_{0}\right) x_{0}, x_{3}\right) & \longmapsto \int \frac{d q_{0} d q_{3}}{\pi^{2}} \frac{i q_{0}|M|}{\left[-\left(q_{0}-i o\right)^{2}+m^{2}\right]\left(q_{3}^{2}+M^{2}\right)} e^{i\left(q_{0} x_{0}-q_{3} x_{3}\right)} \\
& =\vartheta\left(x_{0}\right) \cos m x_{0} e^{-\left|M x_{3}\right|}
\end{aligned}
$$

In a relativistic Lorentz group-compatible form, the two poles show up as the endpoints of an $\mathbf{O}(1,1)$-invariant line singularity $q^{2}=\kappa^{2} \in\left[M^{2}, m^{2}\right]$, characteristic for even-dimensional causal spacetimes,

$$
\begin{aligned}
\int \frac{d^{2} q}{2 \pi} \frac{1}{\left[-(q-i o)^{2}+m^{2}\right]\left[-(q-i o)^{2}+M^{2}\right]} e^{i q x} & =\int_{M^{2}}^{m^{2}} \frac{d \kappa^{2}}{m^{2}-M^{2}} \int \frac{d^{2} q}{2 \pi} \frac{1}{\left[-(q-i o)^{2}+\kappa^{2}\right]^{2}} e^{i q x} \\
& =-\vartheta(x) \int_{M^{2}}^{m^{2}} \frac{d \kappa^{2}}{m^{2}-M^{2}} \frac{\partial \pi \mathcal{J}_{0}(|\kappa x|)}{\partial \kappa^{2}} \\
& =-\vartheta(x) \frac{\pi \mathcal{J}_{0}(|m x|)-\pi \mathcal{J}_{0}(|M x|)}{m^{2}-M^{2}}, \\
\int \frac{d^{2} q}{2 \pi} \frac{i q}{\left[-(q-i o)^{2}+m^{2}\right]\left[-(q-i o)^{2}+M^{2}\right]} e^{i q x} & =\frac{\partial}{\partial x} \int_{M^{2}}^{m^{2}} \frac{d \kappa^{2}}{m^{2}-M^{2}} \int \frac{d^{2} q}{2 \pi} \frac{1}{\left[-(q-i o)^{2}+\kappa^{2}\right]^{2}} e^{i q x} \\
& =\vartheta(x) \frac{x}{|x|} \frac{m \pi \mathcal{J}_{1}(|m x|)-M \pi \mathcal{J}_{1}(|M x|)}{m^{2}-M^{2}},
\end{aligned}
$$

with $\frac{\partial}{\partial x} \mathcal{J}_{0}(|x|)=\frac{x}{2} \frac{\partial}{\partial \frac{I}{x}^{2} 4} \mathcal{J}_{0}(|x|)=-\frac{x}{|x|} \mathcal{J}_{1}(|x|)$. 


\subsection{Even-dimensional Causal Spacetimes}

For even-dimensional spacetime $\mathcal{D}^{2 R}$ in general, the characteristic future functions $\vartheta(x)=$ $\vartheta\left(x_{0}\right) \vartheta\left(x^{2}\right)$ in the translation cone parametrizations have the harmonic analysis with an advanced "energy(-momentum)" distribution $(q-i o)^{2}=\left(q_{0}-i o\right)^{2}-\vec{q}^{2}$ :

$$
\mathbf{S O}_{0}(1,2 R-1): \vartheta(x) \pi\left(\begin{array}{l}
1 \\
x
\end{array}\right)=\int \frac{d^{2 R} q}{2 \pi^{R}} \frac{\Gamma(R)}{\left[-(q-i o)^{2}\right]^{R}}\left(\begin{array}{c}
1 \\
\frac{2 i q R}{-(q-i o)^{2}}
\end{array}\right) e^{i q x} .
$$

The spherical degrees of freedom show up in the order of the pole and in the normalization of the integration $\frac{d^{2 R} q}{2 \pi^{R}}$.

The causal spacetime coefficients with one invariant are

$\mathbf{S O}_{0}(1,2 R-1): \int \frac{d^{2 R} q}{2 \pi^{R}} \frac{\Gamma(R)}{\left[-(q-i o)^{2}+m^{2}\right]^{R}}\left(\begin{array}{c}1 \\ \frac{2 i q R}{-(q-i o)^{2}+m^{2}}\end{array}\right) e^{i q x}=\vartheta(x)\left(\begin{array}{c}1 \\ x\end{array}\right) \pi \mathcal{J}_{0}(|m x|)$.

Even-dimensional spacetime $\mathcal{D}^{2 R}$ with two continuous invariants (real rank $r=2$ ) is represented as the Fourier-transformed product of two "energy-momentum" distributions, one with a simple pole - spherical invariant for dilation (time) $\mathbf{D}(1)$ - and the other one with a pole of order $R=1,2, \ldots-$ hyperbolic invariant for position $\mathcal{Y}^{2 R-1}$, e.g., in the Lorentz scalar and vector functions:

$$
\begin{aligned}
\mathcal{D}^{2 R} \ni \vartheta(x) x & \longmapsto \int \frac{d^{2 R} q}{2 \pi^{R}} \frac{\Gamma(R)}{\left[-(q-i o)^{2}+m^{2}\right]\left[-(q-i o)^{2}+M^{2}\right]^{R}} e^{i q x} \\
& =\int_{0}^{1} d z z^{R-1} \int \frac{d^{2 R} q}{2 \pi^{R}} \frac{\Gamma(1+R)}{\left[-(q-i o)^{2}+(1-z) m^{2}+z M^{2}\right]^{1+R}} e^{i q x} \\
& =\int_{M^{2}}^{m^{2}} \frac{d^{R} \kappa^{2}}{m^{2}-M^{2}} \int \frac{d^{2 R} q}{2 \pi^{R}} \frac{\Gamma(1+R)}{\left[-(q-i o)^{2}+\kappa^{2}\right]^{1+R}} e^{i q x} \\
& =-\vartheta(x) \int_{M^{2}}^{m^{2}} \frac{d^{R} \kappa^{2}}{m^{2}-M^{2}} \frac{\partial \pi \mathcal{J}_{0}(|\kappa x|)}{\partial \kappa^{2}}, \\
\mathcal{D}^{2 R} \ni \vartheta(x) x & \longmapsto \int \frac{d^{2 R} q}{2 \pi^{R}} \frac{i q \Gamma(R)}{\left[-(q-i o)^{2}+m^{2}\right]\left[-(q-i o)^{2}+M^{2}\right]^{R}} e^{i q x} \\
& =-\vartheta(x) \frac{\partial}{\partial x} \int_{M^{2}}^{m^{2}} \frac{d^{R} \kappa^{2}}{m^{2}-M^{2}} \frac{\partial \pi \mathcal{J}_{0}(|\kappa x|)}{\partial \kappa^{2}} \\
& =\int_{M^{2}}^{m^{2}} \frac{d^{R} \kappa^{2}}{m^{2}-M^{2}} \int \frac{d^{2 R} q}{2 \pi^{R}} \frac{i}{2} \frac{\partial}{\partial q} \frac{\Gamma(R)}{\left[-(q-i o)^{2}+\kappa^{2}\right]^{R}} e^{i q x} \\
& =\vartheta(x) \frac{x}{2} \int_{M^{2}}^{m^{2}} \frac{d^{R} \kappa^{2}}{m^{2}-M^{2}} \pi \mathcal{J}_{0}(|\kappa x|) .
\end{aligned}
$$

The spacetime coefficients contain the characteristic dimension-dependent integration with a finite positive spectral $\mathbf{D}(1)$-measure over a line with $\mathbf{O}(1,2 R-1)$-invariants:

$$
\begin{aligned}
& \int_{0}^{1} d z z^{R-1}=\int_{M^{2}}^{m^{2}} \frac{d^{R} \kappa^{2}}{m^{2}-M^{2}} \text { with } \kappa^{2}=(1-z) m^{2}+z M^{2}, \\
& \text { where } d^{R} \kappa^{2}=d \kappa^{2}\left(\frac{m^{2}-\kappa^{2}}{m^{2}-M^{2}}\right)^{R-1}, R=1,2, \ldots
\end{aligned}
$$


Lorentz group $\mathbf{S O}_{0}(1,2 R-1)$-properties, and nontrivial properties with respect to the maximal compact group $\mathbf{S O}(2 R-1)$ for $R \geq 2$, are obtained by derivations $x \sim \frac{\partial}{\partial q}$ and harmonic $\mathbf{S O}_{0}(1,2 R-1)$-polynomials $(x)^{L}$ and $(q)^{L}$.

A pointwise product form for hyperbolic non-relativistic space-time, not compatible with the Lorentz group action looks as follows:

$$
\int \frac{d q_{0} d^{2 R-1} q}{\pi^{1+R}} \frac{i q_{0} \Gamma(R)}{\left[-\left(q_{0}-i o\right)^{2}+m^{2}\right]\left(\vec{q}^{2}+M^{2}\right)^{R}} e^{i\left(q_{0} x_{0}-\vec{q} \vec{x}\right)}=\vartheta\left(x_{0}\right) \cos m x_{0} \frac{e^{-|M| r}}{|M|} .
$$

In the relativistic Lorentz group-compatible form, the representation coefficients for the factor groups arise by projection: The simple pole embeds the representation of the abelian time operations (dilations) $\mathbf{D}(1) \cong \mathbb{R}$ with rank 1 . It is a position distribution of a time representation coefficient: The time projection by integration over position, i.e., for trivial momenta $\int \frac{d^{2 R-1} x}{(2 \pi)^{2 R-1}} e^{-i \vec{q} \vec{x}}=\delta(\vec{q})$, contains a positive-type function $\cos \in L^{\infty}(\mathbb{R})_{+}$for time representations:

$$
\begin{aligned}
\int \frac{d^{2 R-1} x}{(2 \pi)^{2 R}} \int d^{2 R} q \frac{i q}{-(q-i o)^{2}+m^{2}} e^{i q x} & =\int \frac{d q_{0}}{2 \pi} \frac{i q_{0}}{-\left(q_{0}-i o\right)^{2}+m^{2}} e^{i q_{0} x_{0}} \\
& =\vartheta\left(x_{0}\right) \cos m x_{0} .
\end{aligned}
$$

The representation of hyperbolic position $\mathcal{Y}^{2 R-1} \cong \exp \mathbb{R}^{2 R-1}$ with the Lorentz group $\mathbf{S O}_{0}(1,2 R-1)$ as motion group is embedded by a multipole of order $R$. It is a time distribution of a position representation coefficient. The projection on the position hyperboloid with the $\mathbf{S O}_{0}(1,2 R-1)$-coefficients by integration over time, i.e., for trivial energy $\int \frac{d x_{0}}{2 \pi} e^{i q_{0} x_{0}}=\delta\left(q_{0}\right)$, gives a negative energy (bound) state exponential exp $\in L^{2}\left(\mathcal{Y}^{2 R-1}\right)$ for hyperbolic position:

$$
\int \frac{d x_{0}}{\pi} \int \frac{d^{2 R} q}{2 \pi^{R}} \frac{\Gamma(R)}{\left[-(q-i o)^{2}+M^{2}\right]^{R}} e^{i q x}=\int \frac{d^{2 R-1} q}{\pi^{R}} \frac{\Gamma(R)}{x}\left(\vec{q}^{2}+M^{2}\right)^{R} e^{-i \vec{q} \vec{x}}=\frac{e^{-|M| r}}{|M|}
$$

With the $r=2$ (real rank) continuous invariants and the $r_{c}=R-1$ (imaginary rank) discrete invariants of the orthogonal group $\mathbf{S O}(2 R-1)$ with a Cartan torus $\mathbf{S O}(2)^{R-1}$, the acting group $\mathbf{D}(1) \times \mathbf{S O}_{0}(1,2 R-1)$ has rank $r+r_{c}=1+R$.

Unitary relativity $\mathcal{D}^{4}=\mathbf{D}(1) \times \mathcal{Y}^{3}$ with three position dimensions as the minimal nonabelian case has imaginary rank 1 for the compact $\mathbf{S O}(2) \subset \mathbf{S O}(3)$-rotation degrees of freedom. The $\mathcal{D}^{4}$-representation coefficients with two continuous invariants (real rank 2 for noncompact $\left.\mathbf{D}(1) \times \mathbf{S O}_{0}(1,1)\right)$ involve a position-representing dipole, e.g., with Lorentz group vector properties,

$$
\begin{aligned}
\mathbf{G L}(2, \mathbb{C}) / \mathbf{U}(2) \cong \mathbb{R}_{+}^{4} \ni \vartheta(x) x & \longmapsto \int \frac{d^{4} q}{2 \pi^{2}} \frac{i q}{\left[-(q-i o)^{2}+m^{2}\right]\left[-(q-i o)^{2}+M^{2}\right]^{2}} e^{i q x} \\
& \sim \frac{x}{2}\left(\frac{\partial}{\partial \frac{x^{2}}{4}}\right)^{2} \vartheta(x) \pi \mathcal{J}_{0}(|m x|) * \vartheta(x) \pi \mathcal{J}_{0}(|M x|) .
\end{aligned}
$$

With a different motivation and interpretation, a similar "two-point function" for Minkowski spacetime $\mathbb{R}^{(1,3)}$ with Wigner-particle metric and translation ground-state $|0\rangle$ was proposed by Heisenberg in his attempts for a unified field theory of elementary particles (nonlinear spinor theory) with a simple positive metric pole for the baryonic and an ad hoc regularizing indefinite metric dipole related to the leptonic sector. Here, the spacetime function is motivated as coefficient of electroweak spacetime $\mathcal{D}^{4}$ : The simple pole, embedding time (dilation) $\mathbf{D}(1)$-representations $e^{i m x_{0}}$, may be extended and used for a tangent 
Poincaré group representation with Wigner particles. The dipole, embedding Hilbert representations of hyperbolic position $\mathcal{Y}^{3}$ via a negative energy (bound) state exponential $e^{-|M| r}$, has no particle asymptotics by a Minkowski spacetime representation. It will be proposed to describe interactions, not parametrizable by Wigner-particles.

\subsection{Spacetime Hilbert Spaces}

Obviously, the representation coefficients of spacetime $\mathcal{D}^{2 R}$ and the related Hilbert spaces differ from those of its tangent Minkowski spacetime $\mathbb{R}^{(1,2 R-1)}$, used with $R=2$ for particles. They are not Feynman propagators. The free particle Hilbert spaces involve (energy-)momentum functions, in contrast to the non-translational Hilbert spaces with space(-time) functions.

The Hilbert spaces $L^{2}\left(\Omega^{2}\right)$ for Euclidean group representations, i.e., the square integrable functions of the momentum directions on the 2-sphere $\vec{q}^{2}=P^{2}$, are not appropriate for negative energy (bound) state representations with Hilbert space $L^{2}\left(\mathcal{Y}^{3}\right)$, comprising the square integrable functions of Kepler position. Similarily, the Hilbert spaces for Poincaré group representations, e.g., the square integrable functions $L^{2}\left(\mathcal{Y}^{3}\right)$ of the momentum hyperboloid for massive particles (mass shell $q^{2}=m^{2}>0$ ), are not appropriate for electroweak spacetime representations with Hilbert space $L^{2}\left(\mathcal{D}^{4}\right)$, comprising the square integrable functions of causal spacetime. The special relativistic space $L^{2}\left(\mathcal{Y}^{3}\right)$ for Wigner particles has as nonrelativistic subspace $L^{2}\left(\Omega^{2}\right)$ for nonrelativistic free scattering states, e.g., $\frac{\sin P r}{P r}$. The space $L^{2}\left(\mathcal{D}^{4}\right)$ for electroweak spacetime has as position related subspace $L^{2}\left(\mathcal{Y}^{3}\right)$ for hyperbolic position, e.g., $e^{-|B| r}$, as used for the negative energy (bound) states of the Kepler potential:

\begin{tabular}{ll}
\hline free (scattering) & interacting (bound) \\
\hline$L^{2}\left(\Omega^{2}\right)$ & $L^{2}\left(\mathcal{Y}^{3}\right)$ \\
for momenta: & for position: \\
$\vec{q} \in \Omega^{2}, \vec{q}^{2}=P^{2}$, & $e^{\vec{x} \vec{\sigma}} \in \mathcal{Y}^{3}$, \\
$\delta\left(\vec{q}^{2}-P^{2}\right)$ & $\frac{1}{\left(\vec{q}^{2}+B^{2}\right)^{2}}$ \\
$\mathbb{E}^{3} \supset \Omega^{2} \subset \mathcal{Y}^{3} \subset \mathbb{R}^{(1,3)}$ & $\mathcal{Y}^{3} \subset \mathcal{D}^{4}$ \\
$L^{2}\left(\mathcal{Y}^{3}\right)$ & $L^{2}\left(\mathcal{D}^{4}\right)$ \\
for energy-momenta: & for spacetime: \\
$q \in \mathcal{Y}^{3}, q^{2}=m^{2}$, & \\
$\delta\left(q^{2}-m^{2}\right)$ & $e^{x_{0} \mathbf{1}_{2}+\vec{x} \vec{\sigma}} \in \mathcal{D}^{4}$, \\
\hline
\end{tabular}

The Fourier transform of the advanced (retarded) "energy-momentum" integration is, up to the order function, the corresponding principal value integration:

$$
\begin{aligned}
\int \frac{d^{2 R} q}{2 \pi} \frac{\Gamma(1+N)}{\left[-(q \mp i o)^{2}+1\right]^{1+N}} e^{i q x} & =\vartheta\left( \pm x_{0}\right) \int \frac{d^{2 R} q}{\pi} \frac{\Gamma(1+N)}{\left(-q_{\mathrm{P}}^{2}+1\right)^{1+N}} e^{i q x} \\
\int \frac{d^{2 R} q}{\pi} \frac{\Gamma(1+N)}{\left(-q_{\mathrm{P}}^{2}+1\right)^{1+N}} e^{i q x} & =i \epsilon\left(x_{0}\right) \int d^{2 R} q \epsilon\left(q_{0}\right) \delta^{(N)}\left(q^{2}-1\right) e^{i q x} \\
\text { for } N & =0,1,2, \ldots
\end{aligned}
$$


All these spacetime distributions are contained in the Feynman distributions:

$$
\begin{aligned}
\int \frac{d^{2 R} q}{i \pi^{R}} \frac{\Gamma(R-N)}{\left(-q^{2}-i o+1\right)^{R-N}} e^{i q x} & =\left(-\frac{\partial}{\partial \frac{x^{2}}{4}}\right)^{N} 2 \mathcal{K}_{0}\left(\sqrt{-x^{2}+i o}\right), N=0,1, \ldots R-1, \\
\int \frac{d^{2 R} q}{i \pi^{R}} \frac{\Gamma(R+N)}{\left(-q^{2}-i o+1\right)^{R+N}} e^{i q x} & =\left(\frac{x^{2}}{4}\right)^{N}\left(\frac{\partial}{\partial \frac{x^{2}}{4}}\right)^{N} 2 \mathcal{K}_{0}\left(\sqrt{-x^{2}+i o}\right), N=0,1,2, \ldots, \\
\int \frac{d^{2 R} q}{i \pi^{R}} \frac{\Gamma(R)}{\left(-q^{2}-i o+m^{2}\right)^{R}} e^{i q x} & =\int_{m^{2}}^{\infty} \frac{d \kappa^{2}}{\sqrt{\left(\kappa^{2}-m^{2}\right) \kappa^{2}}} e^{-\sqrt{-\kappa^{2} x^{2}+i o}} \\
& =\vartheta\left(x^{2}\right) 2 \mathcal{K}_{0}(i|m x|)+\vartheta\left(-x^{2}\right) 2 \mathcal{K}_{0}(|m x|), \\
2 \mathcal{K}_{0}(i|m x|) & =2 \int_{|m|}^{\infty} \frac{d \kappa}{\sqrt{\kappa^{2}-m^{2}}} e^{-i|\kappa x|} \\
& =-\pi \mathcal{N}_{0}(|m x|)-i \pi \mathcal{J}_{0}(|m x|) .
\end{aligned}
$$

Electroweak spacetime representation coefficients, so far given by causally supported Bessel functions, e.g., $\vartheta(x) x \longmapsto \vartheta(x) \pi \mathcal{J}_{0}(x)$, arise as causally supported part $\vartheta(x) 2 \mathcal{K}_{0}(i|m x|)$ of Fourier transformed Feynman distributions with the unitary contribution, where the exponential is integrated with a positive measure:

$$
\begin{aligned}
R=1,2 & \ldots, L=0,1,2, \ldots: \\
\mathcal{D}^{2 R}: \vartheta(x) x & \longmapsto \vartheta(x) \int \frac{d^{2 R} q}{i \pi^{R}} \frac{\left(m_{L}^{2}-M_{L}^{2}\right) \Gamma(R)}{\left(-q^{2}-i o+m_{L}^{2}\right)\left(-q^{2}-i o+M_{L}^{2}\right)^{R}}(2 i q)^{L} e^{i q x} \\
& =\vartheta(x)(2 \partial)^{L} \int_{M_{L}^{2}}^{m_{L}^{2}} d^{R} \kappa^{2} \int \frac{d^{2 R} q}{i \pi^{R}} \frac{\Gamma(1+R)}{\left(-q^{2}-i o+\kappa^{2}\right)^{1+R}} e^{i q x} \\
& =\vartheta(x)(x)^{L}\left(\frac{\partial}{\partial \frac{x^{2}}{4}}\right)^{L} \int_{M_{L}^{2}}^{m_{L}^{2}} d^{R} \kappa^{2} \int_{|\kappa|}^{\infty} \frac{d \mu}{\sqrt{\left(\mu^{2}-\kappa^{2}\right)^{3}}} e^{-i \mu|x|} \\
\left(\partial^{2}+m_{L}^{2}\right)\left(\partial^{2}+\right. & \left.M_{L}^{2}\right)^{R} \int \frac{d^{2 R} q}{\pi^{R}} \frac{\Gamma(R)(2 i q)^{L}}{\left(-q^{2}-i o+m_{L}^{2}\right)\left(-q^{2}-i o+M_{L}^{2}\right)^{R}} e^{i q x} \\
& =\Gamma(R)(4 \pi)^{R}(2 \partial)^{L} \delta(x) .
\end{aligned}
$$

Multipoles in the characteristic measure $\frac{d^{2 R-1} q}{\pi^{R}} \frac{\Gamma(R)}{\left(\vec{q}^{2}+M^{2}\right)^{R}}$ with $R \geq 2$ do not serve as cutoff as in Minkowski spacetime particle theories, but implement nonflat hyperbolic position with nonabelian Lorentz group and the related negative energy (bound) state exponential. They cannot be connected with simple pole Wigner-particle propagators; the position related hyperbolic invariant (curvature) $M^{2}$ cannot be used as particle mass, i.e., as an invariant for tangent translations $\mathbb{R}^{(1,2 R-1)}$. Multipoles parametrize interactions, "off-shell" and causally supported, and, possibly, can be related to strong interactions, usually implemented by SU(3)-colored quark and gluon degrees of freedom, postulated to be confined, i.e., without translation action (no Wigner-particles). The embedded (eigen)time (dilation) representations with $\frac{d q_{0}}{i \pi} \frac{1}{-q_{0}^{2}-i o+m^{2}}$ (simple poles) can be extended to Minkowski translations representation coefficients for time related spherical invariants $m^{2}$, i.e. to particle degrees of freedom. Harmonic $\mathbf{O}(1,2 R-1)$-polynomials $q_{0}^{L},(\vec{q})^{L} \hookrightarrow(q)^{L}$ implement both energy- and rotation $\mathbf{O}(2 R-1)$-dependence. 
Both factors come, in the product of the $\mathbf{D}(1) \times \mathcal{Y}^{2 R-1}$-contributions, with a residual normalization $\frac{d^{2 R} q}{i \pi^{R}}$. There remains a dilation line integration over $\mathbf{O}(1,2 R-1)$-invariants with $d^{R} \kappa^{2}=d \kappa^{2}\left(\frac{m^{2}-\kappa^{2}}{m^{2}-M^{2}}\right)^{R-1}$. For $(q)^{L}$ with $L=0,1,2$ the representation coefficients are functions, for $L \geq 3$ one obtains distributions proper. More on the normalization ahead.

\section{Normalized Spacetime Representations}

The representation normalizations for position $\mathcal{Y}^{2 R-1}$ and spacetime $\mathcal{D}^{2 R}$ with nonabelian motion groups for $R \geq 2$ determine the continuous invariants for noncompact Cartan subgroups $\mathbf{D}(1) \cong \mathbf{S O}_{0}(1,1)$, e.g., for dilations or translations, by the discrete invariants for compact Cartan subgroups $\mathbf{U}(1) \cong \mathbf{S O}(2)$, e.g., for spin or isospin rotations. For odd-dimensional spaces, the noncompact invariants at point singularities for "energies", "momenta" or masses are related by rational functions to the discrete numbers (Plancherel multiplicities) for the compact invariants, whereas even-dimensional spaces with line singularities employ logarithms for this connection.

The measures of the odd- and even-dimensional maximally symmetric spacetimes are both normalizable by the representation invariant:

$$
\begin{aligned}
\mathbf{O}(t, 1+2 R-t): & \int \frac{d^{1+2 R} q}{i^{t} \pi^{1+R}} \frac{\Gamma(1+R)}{\left(-q^{2}-i o+m^{2}\right)^{1+R}}=\frac{1}{|m|}, \\
\mathbf{O}(t, 2 R-t) & : \int \frac{d^{2 R} q}{i^{t} \pi^{R}} \frac{\Gamma(1+R)}{\left(-q^{2}-i o+m^{2}\right)^{1+R}}=\int_{|m|}^{\infty} \frac{d \kappa}{\sqrt{\left(\kappa^{2}-m^{2}\right)^{3}}}=\frac{1}{m^{2}},
\end{aligned}
$$

which can be taken as intrinsic unit.

The simplest example is the normalization of the time representation coefficients with the energy as intrinsic unit:

$$
\mathbf{D}(1): x_{0} \longmapsto \int \frac{d q_{0}}{i \pi} \frac{1}{-q_{0}^{2}-i o+E^{2}} e^{i q_{0} x_{0}}=\frac{e^{-i\left|E x_{0}\right|}}{|E|},
$$

For odd-dimensional hyperbolic position $\mathcal{Y}^{2 R-1} \cong \mathbf{O}(1,2 R-1) / \mathbf{O}(2 R-1)$ with measure $\frac{d^{2 R-1} q}{\pi^{R}} \frac{\Gamma(R)}{\left(\vec{q}^{2}+1\right)^{R}}$ and harmonic $\mathbf{O}(2 R)$-polynomials with $p=\frac{1}{\vec{q}^{2}+1}\left(\begin{array}{c}1-\vec{q}^{2} \\ 2 \vec{q}\end{array}\right) \in$ $\Omega^{2 R-1}$, e.g., for 3-position as represented by the atomic spectrum, with the representation coefficients

$$
\begin{aligned}
R=1,2, \ldots, \mathcal{Y}^{2 R-1}: \vec{x} & \longmapsto \int \frac{d^{2 R-1} q}{\pi^{R}} \frac{\Gamma(R)}{\left(\vec{q}^{2}+B_{L}^{2}\right)^{R}} \frac{(2 \vec{q})^{L}}{\left(\vec{q}^{2}+B_{L}^{2}\right)^{L}} e^{i \vec{q} \vec{x}} \\
& =\frac{\Gamma(R)}{\Gamma(R+L)}(i \vec{x})^{L} \frac{e^{-\left|B_{L}\right| r}}{\left|B_{L}\right|},
\end{aligned}
$$

compact and noncompact invariants are not independent. The product normalization with the representation distributions for the harmonic $\mathbf{O}(2 R-1)$-polynomials:

$$
\int \frac{d^{2 R-1} q}{(2 \pi)^{2 R-1}}(\vec{q})^{L} e^{i \vec{q} \vec{x}}=(-i \vec{\partial})^{L} \delta(\vec{x}),
$$


determines the hyperbolic invariants $\left|B_{L}\right|$ for a Cartan subgroup $\mathbf{S O}_{0}(1,1) \subseteq \mathcal{Y}^{2 R-1}$ in a reciprocity with the compact invariants for a Cartan subgroup $\mathbf{S O}(2) \subset \mathbf{S O}(2 R-1), R \geq 2$ :

$$
\begin{aligned}
\left(\mathbf{1}_{2 R-1}\right)^{L} & =\int \frac{d^{2 R-1} q}{\pi^{R}} \frac{\Gamma(R)}{\left(\vec{q}^{2}+B_{L}^{2}\right)^{R}} \frac{(2 \vec{q})^{L}}{\left(\vec{q}^{2}+B_{L}^{2}\right)^{L}} \otimes(\vec{q})^{L^{\prime}} \\
& =\delta^{L L^{\prime}} \frac{\Gamma(R) \Gamma(1+L)}{\Gamma(R+L)} \frac{1}{\left|B_{L}\right|}\left(\mathbf{1}_{2 R-1}\right)^{L}, \\
R=1,2, \ldots, \frac{1}{\left|B_{L}\right|} & =\left(\begin{array}{c}
R+L-1 \\
L
\end{array}\right)=\operatorname{dim}_{\mathbb{C}} \bigvee \mathbb{C}^{R},
\end{aligned}
$$

e.g., $\frac{1}{B_{L}^{2}}=(1+L)^{2}$ in the atomic spectrum for $\mathcal{Y}^{3}$. The representation invariant $\left|B_{0}\right|$ for the scalar coefficients can be used as intrinsic unit, e.g., the Rydberg energy $\underline{B_{0}^{2}}=m_{R} c^{2}$ in $\frac{m_{R} c^{2}}{B_{L}^{2}}=(1+L)^{2}$. For abelian position $\mathcal{Y}^{1}=\mathbf{S O}_{0}(1,1)$ without rotations there is no $L$-dependence, $\left|B_{L}\right|=\left|B_{0}\right|$.

\subsection{Logarithmic Mass Ratios for Indefinite Spacetimes}

The characteristic measure for an indefinite even-dimensional manifold with odd-odd submanifolds involves, in addition to the residual normalization, an integration over both invariants (units) in the embedded characteristic submanifold measures:

$$
\begin{aligned}
\mathbf{O}(1 & \left.+2 R_{t}, 1+2 R_{s}\right): \int \frac{d^{2\left(1+R_{t}+R_{s}\right)}}{\pi^{1+R_{t}+R_{s}}} \frac{\Gamma\left(1+R_{t}\right)}{\left(-q^{2}-i o+m^{2}\right)^{1+R_{t}}} \frac{\Gamma\left(1+R_{s}\right)}{\left(-q^{2}-i o+M^{2}\right)^{1+R_{s}}} \\
& =\int_{0}^{1} d z(1-z)^{R_{t}} z^{R_{s}} \int \frac{d^{2\left(1+R_{t}+R_{s}\right)} q}{\pi^{1+R_{t}+R_{s}}} \frac{\Gamma\left(2+R_{t}+R_{s}\right)}{\left(-q^{2}-i o+(1-z) m^{2}+z M^{2}\right)^{2+R_{t}+R_{s}}} \\
& =\int_{0}^{1} d z \frac{(1-z)^{R_{t}} z^{R_{s}}}{(1-z) m^{2}+z M^{2}}=\frac{1}{m^{2}-M^{2}} \int_{M^{2}}^{m^{2}} \frac{d \kappa^{2}}{\kappa^{2}} \frac{\left(\kappa^{2}-M^{2}\right)^{R_{t}}\left(m^{2}-\kappa^{2}\right)^{R_{s}}}{\left(m^{2}-M^{2}\right)^{R_{t}+R_{s}} .}
\end{aligned}
$$

A spacetime coefficient with two continuous invariants (units), characterizing time and position $\left(E^{2}, B^{2}\right) \leftrightarrow\left(\mathbf{D}(1), \mathcal{Y}^{2 R-1}\right)$, is, for the nonrelativistic case, in the $\mathbf{O}(1) \times \mathbf{O}(2 R-$ 1)-compatible, but Lorentz group-incompatible form with the two residual integrations $\int \frac{d q_{0}}{i \pi}$ and $\int \frac{d^{2 R-1} q}{\pi^{R}}$ normalized by the product of the two invariants:

$$
\mathbf{O}(1) \times \mathbf{O}(2 R-1): \int \frac{d q_{0}}{i \pi} \frac{1}{-q_{0}^{2}-i o+E^{2}} \int \frac{d^{2 R-1} q}{\pi^{R}} \frac{\Gamma(R)}{\left(\vec{q}^{2}+B^{2}\right)^{R}}=\frac{1}{|E||B|} .
$$

In the Lorentz group $\mathbf{O}(1,2 R-1)$-compatible form with embedded time and position $\left(m^{2}, M^{2}\right) \leftrightarrow\left(\mathbf{D}(1), \mathcal{Y}^{2 R-1}\right)$,

$$
\begin{aligned}
\mathbf{O}(1,2 R & -1): \int \frac{d^{2 R} q}{i \pi^{R}} \frac{\Gamma(R)}{\left(-q^{2}-i o+m^{2}\right)\left(-q^{2}-i o+M^{2}\right)^{R}} \\
& =\frac{1}{m^{2}-M^{2}} \int_{M^{2}}^{m^{2}} d^{R} \kappa^{2} \int \frac{d^{2 R} q}{i \pi^{R}} \frac{\Gamma(1+R)}{\left(-q^{2}-i o+\kappa^{2}\right)^{1+R}} \\
& =\int_{0}^{1} d z \frac{z^{R-1}}{(1-z) m^{2}+z M^{2}}=\frac{1}{m^{2}-M^{2}} \int_{M^{2}}^{m^{2}} \frac{d^{R} \kappa^{2}}{\kappa^{2}}=-\frac{1}{m^{2}-M^{2}} \log _{R} \frac{M^{2}}{m^{2}},
\end{aligned}
$$

the representation normalization connects both invariants by a characteristic line integration $\int_{M^{2}}^{m^{2}} \frac{d^{R} \kappa^{2}}{\kappa^{2}}$ for a finite dilation measure. In the case of different invariants, $m^{2} \neq M^{2}$, i.e., for 
not maximally symmetric spacetimes, a multiplication with the difference $\int_{M^{2}}^{m^{2}} d \kappa^{2}=m^{2}-$ $M^{2}$ of the two invariants in the integration length defines the characteristic dimensionless "energy-momentum" measures

$$
\text { for a Fourier analysis of } \mathcal{D}^{2 R}: \frac{d^{2 R} q}{i \pi^{R}} \frac{\left(m^{2}-M^{2}\right) \Gamma(R)}{\left(-q^{2}-i o+m^{2}\right)\left(-q^{2}-i o+M^{2}\right)^{R}}
$$

If the minimal abelian case,

$$
\frac{1}{i \pi} \frac{m^{2}-M^{2}}{\left(-q^{2}-i o+m^{2}\right)\left(-q^{2}-i o+M^{2}\right)}=\delta\left(q^{2}-M^{2}\right)-\delta\left(q^{2}-m^{2}\right)+\ldots,
$$

is taken with an additive decomposition for a flat spacetime $\mathbb{R}^{(1,1)}$-metric, the on-shell contributions of the simple poles have opposite metric (no Hilbert representation of the Poincaré group). Such an additive decomposition is not appropriate for Cartan spacetime $\mathcal{D}^{2}=\mathbf{D}(1) \times \mathbf{S O}_{0}(1,1)$ and, in general, for $\mathcal{D}^{2 R}=\mathbf{D}(1) \times \mathcal{Y}^{2 R-1}$ where the coefficients $\frac{1}{-q^{2}-i o+m^{2}} \cdot \frac{\Gamma(R)}{\left(-q^{2}-i o+M^{2}\right)^{R}}$ represent a product space.

The characteristic $\mathcal{D}^{2 R}$-measures are normalized by the logarithmic $R$-tails with the dimensionless ratio $\mu^{2}=\frac{M^{2}}{m^{2}}>0$ of the hyperbolic and spherical invariant:

$$
\begin{aligned}
R=1,2, \ldots:-\log _{R} \mu^{2} & =\int_{0}^{1} d z \frac{\left(1-\mu^{2}\right) z^{R-1}}{1-\left(1-\mu^{2}\right) z}=\int_{\mu^{2}}^{1} \frac{d^{R} \kappa^{2}}{\kappa^{2}}=\int_{\mu^{2}}^{1} \frac{d \kappa^{2}}{\kappa^{2}}\left(\frac{1-\kappa^{2}}{1-\mu^{2}}\right)^{R-1} \\
& =-\frac{1}{\left(1-\mu^{2}\right)^{R-1}}\left[\log \mu^{2}+\sum_{k=1}^{R-1} \frac{\left(1-\mu^{2}\right)^{k}}{k}\right] \\
& =\sum_{k=R}^{\infty} \frac{\left(1-\mu^{2}\right)^{k-(R-1)}}{k}=- \begin{cases}\log \mu^{2}, & R=1, \\
\frac{\log \mu^{2}}{1-\mu^{2}}+1, & R=2, \\
\cdots & \frac{1}{1-\mu^{2}} \log _{R} \mu^{2}-\frac{1}{R} .\end{cases}
\end{aligned}
$$

With the factor $m^{2}-M^{2} \sim 1-\mu^{2}$, the logarithmic tails are positive for $\mu^{2}<1$, i.e., for a smaller hyperbolic invariant $M^{2}<m^{2}$, and negative for $\mu^{2}>1$, i.e., for a smaller spherical invariant $M^{2}>m^{2}$, and monotoneously decreasing:

$-\frac{\log _{R} \mu^{2}}{1-\mu^{2}}=\int_{0}^{1} d z \frac{z^{R-1}}{1-\left(1-\mu^{2}\right) z}>0,-\frac{\partial}{\partial \mu^{2}} \log _{R} \mu^{2}=-\int_{0}^{1} d z \frac{z^{R-1}}{\left[1-\left(1-\mu^{2}\right) z\right]^{2}} \leq 0$,

with the following expansions and special values:

$$
-\log _{R} \mu^{2}= \begin{cases}-\log \mu^{2}-\mathbf{S U} M_{k=1}^{R-1} \frac{1}{k}+\ldots, & \mu^{2} \ll 1 \\ 0, & \mu^{2}=1 \\ -\frac{1}{R-1}+\ldots, R \neq 1, & \mu^{2} \gg 1\end{cases}
$$




\subsection{Multiplicities for External Degrees of Freedom}

For the Lorentz group-compatible representations of even-dimensional spacetime $\mathcal{D}^{2 R}=$ $\mathbf{D}(1) \times \mathcal{Y}^{2 R-1}$ with harmonic $\mathbf{O}(1,2 R-1)$-polynomials,

$$
\begin{aligned}
R=1,2, \ldots, \mathcal{D}^{2 R}: \vartheta(x) x & \longmapsto \vartheta(x) d^{L}(x) \\
L=0,1, \ldots, & \\
d^{L}(x) & =\int \frac{d^{2 R} q}{i \pi^{R}} \frac{\left(m_{L}^{2}-M_{L}^{2}\right) \Gamma(R)}{\left(-q^{2}-i o+m_{L}^{2}\right)\left(-q^{2}-i o+M_{L}^{2}\right)^{R}}(2 i q)^{L} e^{i q x} \\
& =\int_{M_{L}^{2}}^{m_{L}^{2}} d^{R} \kappa^{2} \int \frac{d^{2 R} q}{i \pi^{R}} \frac{\Gamma(1+R)}{\left(-q^{2}-i o+\kappa^{2}\right)^{1+R}}(2 i q)^{L} e^{i q x} \\
& =(2 \partial)^{L} d^{0}\left(x^{2}\right)=(x)^{L}\left(\frac{\partial}{\partial \frac{x^{2}}{4}}\right)^{L} d^{0}\left(x^{2}\right),
\end{aligned}
$$

the inverse spacetime $\mathcal{D}^{2 R}$-derivatives with the dual ("inverse") harmonic dependence, i.e., the spacetime distributions:

$$
\begin{aligned}
\frac{1}{(\partial)^{L}} \sim \int \frac{d^{2 R} q}{i \pi^{R}} \frac{(i q)^{L}}{\left(-q^{2}-i o\right)^{L}} e^{i q x} & =\frac{1}{\Gamma(L)}(\partial)^{L} \frac{\Gamma(R-L)}{\left(-\frac{x^{2}}{4}+i o\right)^{R-L}} \\
& =\frac{1}{\Gamma(L)}\left(\frac{x}{2}\right)^{L} \frac{\Gamma(R)}{\left(-\frac{x^{2}}{4}+i o\right)^{R}},
\end{aligned}
$$

are used for the external multiplicity factors in the product normalizations:

$$
\begin{aligned}
\left(\mathbf{1}_{2 R}\right)^{L} & =\int \frac{d^{2 R} q}{i \pi^{R}} \frac{\left(m_{L}^{2}-M_{L}^{2}\right) \Gamma(R)}{\left(-q^{2}-i o+m_{L}^{2}\right)\left(-q^{2}-i o+M_{L}^{2}\right)^{R}}(2 i q)^{L} \otimes \frac{(i q)^{L^{\prime}}}{\left(-q^{2}-i o\right)^{L^{\prime}}} \\
& =\delta^{L L^{\prime}} \frac{\Gamma(R) \Gamma(1+L)}{\Gamma(R+L)} \int_{M_{L}^{2}}^{m_{L}^{2}} d^{R} \kappa^{2} \int \frac{d^{2 R} q}{i \pi^{R}} \frac{\Gamma(1+R)}{\left(-q^{2}-i o+\kappa^{2}\right)^{1+R}}\left(\mathbf{1}_{2 R}\right)^{L} \\
& =-\delta^{L L^{\prime}} \frac{\Gamma(R) \Gamma(1+L)}{\Gamma(R+L)} \log _{R} \frac{M_{L}^{2}}{m_{L}^{2}}\left(\mathbf{1}_{2 R}\right)^{L} .
\end{aligned}
$$

The line singularity normalizations give a compact-noncompact reciprocity for the logarithmic tails of the ratio of the two noncompact invariants:

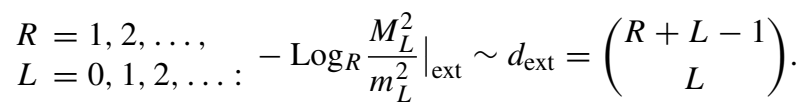

This normalization with the external multiplicities has to be supplemented by the normalization with respect to the internal degrees of freedom.

\subsection{Multiplicities for Internal Degrees of Freedom}

A representation of four-dimensional electroweak spacetime $\mathbb{D}(2) \cong \mathbf{G L}(2, \mathbb{C}) / \mathbf{U}(2)$ uses, for external operations with Lorentz $\mathbf{S L}(2, \mathbb{C})$ and internal operations with isospin $\mathbf{S U}(2)$, a subrepresentation of the bi-regular $\mathbf{G L}(2, \mathbb{C})$-representation $\mathbf{G L}(2, \mathbb{C}) \times \mathbf{U}(2) \longrightarrow$ $\mathbf{G L}(W) \times \mathbf{G L}(V)$ in a product group. The nonscalar representations start for $\mathbf{G L}(2, \mathbb{C})$ with the $(2 \times 2)$-Weyl representation $q \sim \sigma^{j} q_{j}=\left(\begin{array}{cc}q_{0}+q_{3} & q_{1}-i q_{2} \\ q_{1}+i q_{2} & q_{0}-q_{3}\end{array}\right)$ with unit $\sigma^{0}=\mathbf{1}_{2}$ 
and its internal $\mathbf{U}(2)$-partner, the Pauli $(2 \times 2)$-representation with unit $\mathbf{1}_{2}$. In general, the irreducible harmonic $\mathbf{S L}(2, \mathbb{C})$-dependence $(q)^{L}$ from the totally symmetric powers of $W_{1} \cong \mathbb{C}^{2}$ comes with the corresponding unrestricted tensor powers of $V_{1} \cong \mathbb{C}^{2}$ with decomposable $\mathbf{S U}(2)$-representations:

$$
\begin{aligned}
& \mathcal{D}^{4}: \vartheta(x) x \longmapsto \vartheta(x) \int \frac{d^{4} q}{i \pi^{2}} \frac{\left(m_{L}^{2}-M_{L}^{2}\right)}{\left(-q^{2}-i o+m_{L}^{2}\right)\left(-q^{2}-i o+M_{L}^{2}\right)^{2}}(2 i q)^{L} \times \mathbf{1}_{2^{L}} e^{i q x}, \\
& \text { with } \begin{cases}\text { external (spin): } & W_{L} \cong \bigvee^{L} \mathbb{C}^{2} \cong \mathbb{C}^{1+L}, \quad(q)^{L} \in \mathbf{A} \mathbf{L}(1+L, \mathbb{C}), \\
\text { internal (isospin): } & V_{L} \cong \bigotimes^{L} \mathbb{C}^{2} \cong \mathbb{C}^{2^{L}}, \quad \otimes^{L} \mathbf{1}_{2}=\mathbf{1}_{2^{L}} \in \mathbf{U}\left(2^{L}\right) .\end{cases}
\end{aligned}
$$

$I=\frac{L}{2}$ is the maximal isospin in $\mathbb{C}^{2^{L}}$ :

\begin{tabular}{lllll}
\hline maximal $I$ & $\frac{1}{2}$ & 1 & $\frac{3}{2}$ & $\cdots$ \\
\hline $\mathbb{C}^{2^{2 I}}$ & $\mathbb{C}^{2}$ & $\mathbb{C}^{4}=\mathbb{C}^{3} \oplus \mathbb{C}$ & $\mathbb{C}^{8}=\mathbb{C}^{4} \oplus 2 \times \mathbb{C}^{2}$ & $\cdots$ \\
\hline
\end{tabular}

The normalization of electroweak spacetime $\mathbf{G L}(2, \mathbb{C}) / \mathbf{U}(2)$ by the $\mathbf{G L}(2, \mathbb{C}) \times \mathbf{U}(2)$ unit takes into account the internal multiplicity $d=2^{L}$,

$$
\begin{aligned}
\left(\mathbf{1}_{4}\right)^{L} \times\left(\mathbf{1}_{d} \otimes \mathbf{1}_{d}\right)= & \int \frac{d^{4} q}{i \pi^{2}} \frac{\left(m_{L}^{2}-M_{L}^{2}\right)}{\left(-q^{2}-i o+M_{L}^{2}\right)^{2}\left(-q^{2}-i o+m_{L}^{2}\right)}(2 i q)^{L} \\
& \times \mathbf{1}_{d} \otimes \frac{(i q)^{L} \times \mathbf{1}_{d}}{\left(-q^{2}-i o\right)^{L}} \\
= & \frac{\Gamma(1+L)}{\Gamma(2+L)} \int_{M_{L}^{2}}^{m_{L}^{2}} d^{2} \kappa^{2} \int \frac{d^{4} q}{i \pi^{2}} \frac{2}{\left(-q^{2}-i o+\kappa^{2}\right)^{3}}\left(\mathbf{1}_{4}\right)^{L} \times\left(\mathbf{1}_{d} \otimes \mathbf{1}_{d}\right) \\
& \stackrel{\text { Fierz }}{=}-\frac{1}{1+L} \log _{2} \frac{M_{L}^{2}}{m_{L}^{2}}\left(\mathbf{1}_{4}\right)^{L} \times \frac{\mathbf{1}_{d} \otimes \mathbf{1}_{d}}{d}+\ldots,
\end{aligned}
$$

from the Fierz recoupling $\left(\mathbf{1}_{d}\right)_{\alpha}^{\beta}\left(\mathbf{1}_{d}\right)_{\gamma}^{\delta}=\frac{1}{d}\left(\mathbf{1}_{d}\right)_{\alpha}^{\delta}\left(\mathbf{1}_{d}\right)_{\gamma}^{\beta}+\ldots$, necessary for the decomposition of the product representations. The internal Fierz recoupling is the analogue to the external "energy-momenta" recoupling $\left(q_{1}, q_{2}\right) \leftrightarrow\left(q_{1}+q_{2}, q_{1}-q_{2}\right)$ into relative and center-of-mass contributions,

Pauli matrices $\left(\tau^{a}\right)_{a=1}^{d^{2}-1} \in \mathbf{A} \mathbf{L}(d, \mathbb{C}), d=2,3, \ldots$,

$$
\left(\begin{array}{l}
\mathbf{1}_{d} \otimes \mathbf{1}_{d} \\
\tau^{a} \otimes \tau^{a}
\end{array}\right) \stackrel{\text { Fierz }}{=} F\left(\begin{array}{l}
\mathbf{1}_{d} \otimes \mathbf{1}_{d} \\
\tau^{a} \otimes \tau^{a}
\end{array}\right), F=\frac{1}{d}\left(\begin{array}{cc}
1 & 1 \\
d^{2}-1 & -1
\end{array}\right), F \circ F=\mathbf{1}_{2} .
$$

The rearranged unit $\mathbf{1}_{d} \otimes \mathbf{1}_{d}$ is normalized with the internal dimension $d$.

Altogether one obtains the external-internal normalization conditions with the noncompact-compact reciprocity,

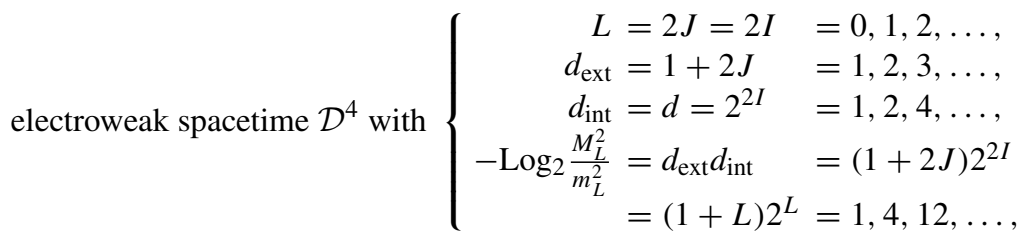


and the association of spacetime $\mathcal{D}^{4}$-coefficients and numerical mass values for $L=0,1,2$ :

\begin{tabular}{llll}
\hline$(J, I)=\left(\frac{L}{2}, \frac{L}{2}\right)$ & $\frac{(2 i q)^{2 J} \times \mathbf{1}_{2} 2 I}{\left(-q^{2}-i o+M_{L}^{2}\right)^{2}\left(-q^{2}-i o+m_{L}^{2}\right)}$ & $-\log _{2} \frac{M_{L}^{2}}{m_{L}^{2}}=(1+2 J) 2^{2 I}$ & $\frac{M_{L}^{2}}{m_{L}^{2}}$ \\
\hline$(0,0)$ & $\frac{1}{\left(-q^{2}-i o+M_{0}^{2}\right)^{2}\left(-q^{2}-i o+m_{0}^{2}\right)}$ & $-\log _{2} \frac{M_{0}^{2}}{m_{0}^{2}}=1$ & $\frac{M_{0}^{2}}{m_{0}^{2}} \sim e^{-2}$ \\
$\left(\frac{1}{2}, \frac{1}{2}\right)$ & $\frac{2 i q \times \mathbf{1}_{2}}{\left(-q^{2}-i o+M_{1}^{2}\right)^{2}\left(-q^{2}-i o+m_{1}^{2}\right)}$ & $-\log _{2} \frac{M_{1}^{2}}{m_{1}^{2}}=4$ & $\sim 10^{-1}$ \\
$(1,1)$ & $\frac{\left(-4 q \otimes q+\eta q^{2}\right) \times \mathbf{1}_{4}}{\left(-q^{2}-i o+M_{2}^{2}\right)^{2}\left(-q^{2}-i o+m_{2}^{2}\right)}$ & $-\log _{2} \frac{M_{2}^{2}}{m_{2}^{2}}=12$ & $\frac{M_{1}^{2}}{m_{1}^{2}} \sim e^{-5}$ \\
& & & $\frac{M_{2}^{2}}{m_{2}^{2}} \sim e^{-13}$ \\
& & & $\sim 10^{-5.6}$ \\
\hline
\end{tabular}

In analogy to the condition for hyperbolic position $\mathcal{Y}^{3}$, one may call this an equipartion of the logarithmic tail to the external and internal dimensions of the electroweak spacetime $\mathcal{D}^{4}$-representations.

\section{Particles and Interactions of Spacetime}

An attempt will be given to relate the mathematical representation structures above with experimentally observed physical structures: Electroweak spacetime $\mathcal{D}^{4} \cong \mathbf{G L}(2, \mathbb{C}) / \mathbf{U}(2)$, parametrizable by strict Minkowski spacetime future, is fundamentally realized by irreducible spinor-isospinor representations for the external-internal group $\mathbf{G L}(2, \mathbb{C}) \times \mathbf{U}(2)$ (without indices $\frac{M_{1}^{2}}{m_{1}^{2}}=\frac{M^{2}}{m^{2}}$ ) with the representation coefficient:

$$
\begin{aligned}
(J, I)=\left(\frac{1}{2}, \frac{1}{2}\right): \mathcal{D}^{4} \ni \vartheta(x) x & \longmapsto \vartheta(x) \int \frac{d^{4} q}{i \pi^{2}} \frac{\left(m^{2}-M^{2}\right) 2 i q \times \mathbf{1}_{2}}{\left(-q^{2}-i o+m^{2}\right)\left(-q^{2}-i o+M^{2}\right)^{2}} e^{i q x} \\
\text { with }-\log _{2} \frac{M^{2}}{m^{2}} & =(1+2 J)(1+2 I)=4 \Rightarrow \frac{M^{2}}{m^{2}} \sim e^{-5} \sim 10^{-2.2}
\end{aligned}
$$

This spacetime convolution product can be interpreted as a position $\mathcal{Y}^{3}$-distribution of a time $\mathbf{D}(1)$-representation by a pole with spherical invariant $m^{2}$ and as a time $\mathbf{D}(1)$ distribution of a position $\mathcal{Y}^{3}$-representation by a dipole with hyperbolic invariant $M^{2}$. A spectral decomposition for Poincaré group and Minkowski spacetime representations with a linear combination of simple poles and a dipole would involve positive and negative metric contributions - it is inappropriate here.

In a tangent space extension, the simple pole may give rise to an on-shell Wigner-particle structure for the leptonic sector with a particle field $\mathbf{l}$ :

$$
\begin{aligned}
\mathbf{D}(1) \ni x_{0} \longmapsto \int d q_{0} q_{0} \delta\left(-q_{0}^{2}+m^{2}\right) e^{i q_{0} x_{0}} & \hookrightarrow \int \frac{d^{4} q}{\pi} q \times \mathbf{1}_{2} \delta\left(-q^{2}+m^{2}\right) e^{i q x} \\
& \sim\left\langle\mathbf{I I}^{*}\right\rangle(x),
\end{aligned}
$$


whereas the dipole has no connection with a Wigner-particle structure. It may be related to causally supported strong interactions, usually parametrized by the gluon and the quark fields q, postulated to be confined (never "on-shell”, i.e., no translation representations):

$$
\begin{aligned}
\mathcal{Y}^{3} \ni \vec{x} \longmapsto \int \frac{d^{3} q}{\pi^{2}} \frac{\vec{q}}{\left(\vec{q}^{2}+M^{2}\right)^{2}} e^{-i \vec{q} \vec{x}} & \hookrightarrow \int \frac{d^{4} q}{\pi^{2}} \frac{q \times \mathbf{1}_{2}}{\left(-q_{\mathrm{P}}^{2}+M^{2}\right)^{2}} e^{i q x} \\
& \sim\left\langle\mathbf{q} \mathbf{q}^{*}\right\rangle(x) .
\end{aligned}
$$

In a field language, the representation matrix element can be related to a lepton-quark product field $\boldsymbol{\chi}=\mathbf{l} \otimes \mathbf{q}$ with $\left\langle\mathbf{l} \otimes \mathbf{q q}^{*} \otimes \mathbf{l}^{*}\right\rangle(x) \sim\left\langle\mathbf{I I}^{*}\right\rangle *\left\langle\mathbf{q} \mathbf{q}^{*}\right\rangle(x)$ where the leptonic degrees of freedom $\mathbf{l}$ embed time $\mathbf{D}(1)$ and the quark degrees of freedom $\mathbf{q}$ position $\mathcal{Y}^{3}$.

The SU(3)-color triplet degrees of freedom $\mathbf{1}_{3}$ in a conventional quark-particle flat spacetime formulation $\left\langle\mathbf{q q}^{*}\right\rangle(x) \sim \int \frac{d^{4} q}{\pi^{2}} \frac{q \times \mathbf{1}_{2} \times \mathbf{1}_{3}}{-q^{2}-i o+M^{2}} e^{i q x}$ may be introduced via a "custodian" SU(3) to distinguish lepton and quark degrees of freedom in a Minkowski spacetime interpretation. The color singlet spacetime convolution cube comes with a dipole "energy-momentum" function $\left[\frac{q \times \mathbf{1}_{2} \times \mathbf{1}_{3}}{q^{2}}\right]^{3} \sim \frac{q \times \mathbf{1}_{2}}{\left(q^{2}\right)^{2}}$, more general for $(2 R-1)$-dimensional position $\mathcal{Y}^{2 R-1} \subset \mathcal{D}^{2 R}$ with $\mathbf{S U}(2 R-1)$-color degrees of freedom and an order $R$-pole $\left[\frac{q \times \mathbf{1}_{2 R-1}}{q^{2}}\right]^{2 R-1} \sim \frac{q}{\left(q^{2}\right)^{R}}$. In such an interpretation the "custodian" color group $\mathbf{S U}(2 R-1)$ for a simple pole (usual quarks for $R=2$ ) may be looked at to be a totally antisymmetric $(2 R-1)$-root of a pole of order $R$ with $\bigwedge^{2 R-1} \mathbf{S U}(2 R-1)=\{1\}$, generalizing the cyclotomic groups $\mathbf{I}(2 R-1)=\sqrt[2 R-1]{1}$. The dimensions for position and unitary "custodian" color group are related to each other, e.g., three position dimensions use quark triplets.

In the case of color confinement, a nontrivial color representation is incompatible with a nontrivial translation representation.

A lepton-quark association to the electroweak spacetime representation coefficient leads to a ratio $\frac{M}{m} \sim \frac{1}{12}$ of an average quark "mass" $M$ (hyperbolic or interaction invariant) to an average lepton mass $m$ (spherical or particle invariant). The proliferation of lepton and quark degrees of freedom into different families, e.g., $\mathbf{l} \longrightarrow\{\mathbf{e}, \boldsymbol{\mu}, \boldsymbol{\tau}\}$, may be related to an isospin-degeneracy (breakdown) of the ground state, not discussed here.

The complex infinite-dimensional vector space with the $\mathcal{D}^{4}$-action may be spanned by a basis $\left\{\chi_{A}^{\alpha}(x) \mid A=1,2 ; \alpha=1,2 ; x \in \mathcal{D}^{4}\right\}$ with Weyl spinor-isospinors and the electroweak ground state $\backslash \mathcal{D}$ characterizing coefficient:

$$
\begin{aligned}
x, y \in \mathcal{D}^{4}:\left\langle\mathcal{D} \chi_{A}^{\alpha}(y) \chi_{\beta}^{*}(x+y)\right\rangle \mathcal{D} & \cong\left\langle\chi \chi^{*}\right\rangle(x) \\
& =\vartheta(x) \int \frac{d^{4} q}{i \pi^{2}} \frac{\left(m^{2}-M^{2}\right) 2 i q \times \mathbf{1}_{2}}{\left(-q^{2}-i o+m^{2}\right)\left(-q^{2}-i o+M^{2}\right)^{2}} e^{i q x} .
\end{aligned}
$$

The causally supported spacetime field $\chi$ has no harmonic analysis with respect to the Poincaré group, especially no translations representations and no corresponding equation of motion. The convolution product for $\mathcal{D}^{4}=\mathbf{D}(1) \times \mathcal{Y}^{3}$ has projections to the simple pole particle $\mathbf{D}(1)$ - and dipole interaction $\mathcal{Y}^{3}$-distributions:

$$
\begin{aligned}
\left\langle\chi \chi^{*}\right\rangle(x) & \sim\left\langle\mathbf{I I}^{*}\right\rangle *\left\langle\mathbf{q q} \mathbf{q}^{*}\right\rangle(x), \\
\left(\partial^{2}+M^{2}\right)^{2}\left\langle\chi \chi^{*}\right\rangle(x) & =\vartheta(x) \int \frac{d^{4} q}{i \pi^{2}} \frac{\left(m^{2}-M^{2}\right) 2 i q \times \mathbf{1}_{2}}{-q^{2}-i o+m^{2}} e^{i q x} \sim\left\langle\mathbf{I I}^{*}\right\rangle(x), \\
\left(\partial^{2}+m^{2}\right)\left\langle\chi \chi^{*}\right\rangle(x) & =\vartheta(x) \int \frac{d^{4} q}{i \pi^{2}} \frac{\left(m^{2}-M^{2}\right) 2 i q \times \mathbf{1}_{2}}{\left(-q^{2}-i o+M^{2}\right)^{2}} e^{i q x} \sim\left\langle\mathbf{q q}^{*}\right\rangle(x) .
\end{aligned}
$$


Acknowledgments Open access funding provided by Max Planck Society.

Open Access This article is distributed under the terms of the Creative Commons Attribution 4.0 International License (http://creativecommons.org/licenses/by/4.0/), which permits unrestricted use, distribution, and reproduction in any medium, provided you give appropriate credit to the original author(s) and the source, provide a link to the Creative Commons license, and indicate if changes were made. 